\title{
Implementation of On-the-Fly Doppler Broadening in MCNP5 for Multiphysics Simulation of Nuclear Reactors
}

\section{Fuel Cycle R\&D}

Dr. William Martin University of Michigan

In collaboration with: Los Alamos National Laboratory Argonne National Laboratory

David Pointer, Technical POC Rob Versluis, Federal POC 


\title{
Implementation of On-the-Fly Doppler Broadening in MCNP for Multiphysics Simulation of Nuclear Reactors
}

\author{
Final Report
}

\section{DE-AC07-05ID14517 \\ Project 10-897}

\author{
William R. Martin, PI \\ Gokhan Yesilyurt, Argonne National Laboratory, co-PI \\ Forrest B. Brown, Los Alamos National Laboratory, co-I \\ Scott J. Wilderman, co-I \\ University of Michigan \\ Department of Nuclear Engineering and Radiological Sciences \\ Ann Arbor, MI 48109-2104
}

November 2012 


\section{Table of Contents}

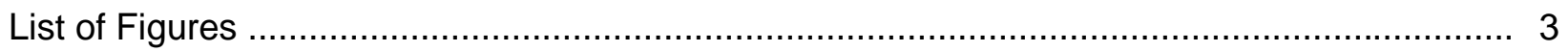

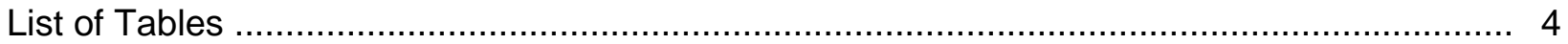

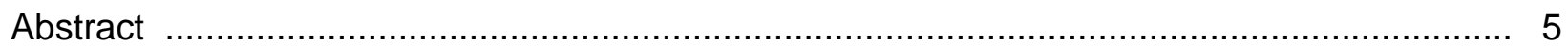

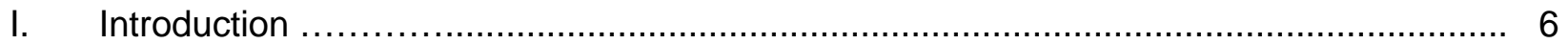

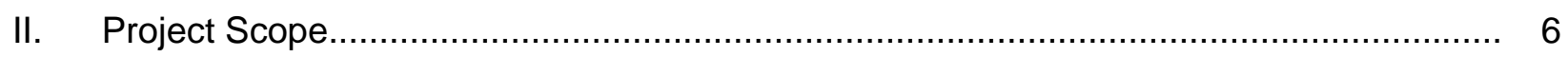

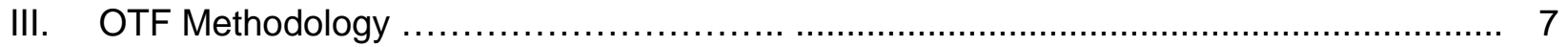

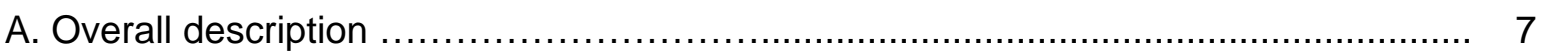

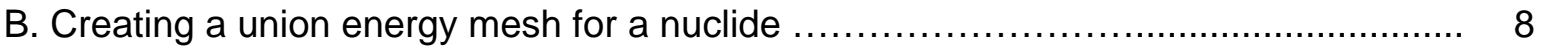

C. Determining Doppler-broadened cross sections on the union energy grid ............ 10

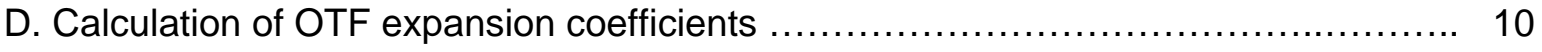

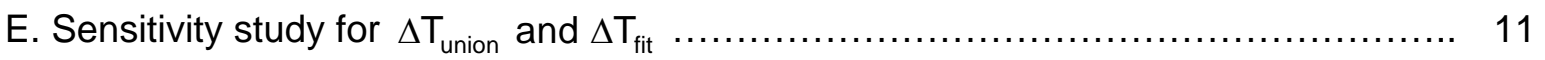

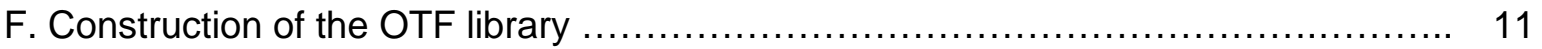

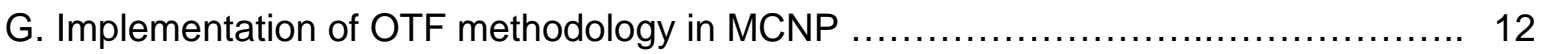

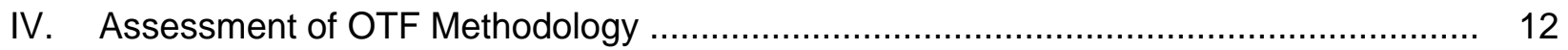

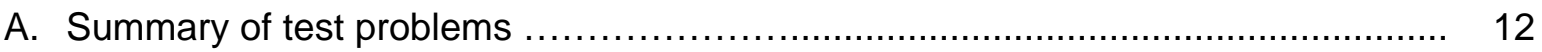

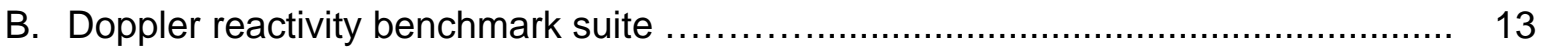

C. Doppler reactivity benchmark suite with radial temperature dependence ............. 14

D. Full core VHTR with imposed temperature distribution ..................................... 16

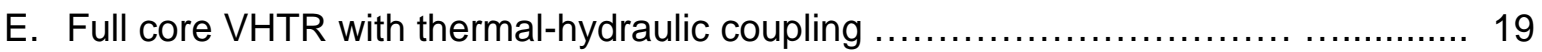

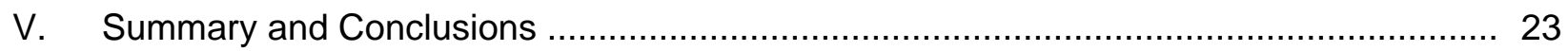

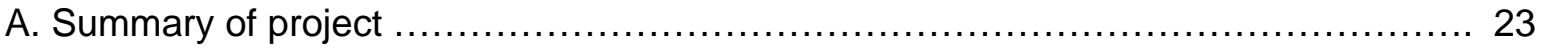

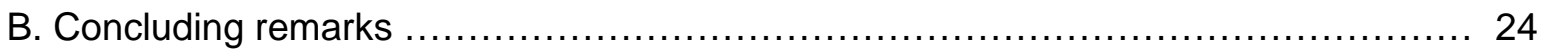

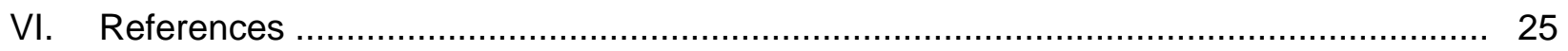

VII. Students Supported by the Contract ............................................................. 25

VIII. Publications Directly Related to the Contract .................................................... 25

IX. Reports and Other Publications ….................................................................. 26

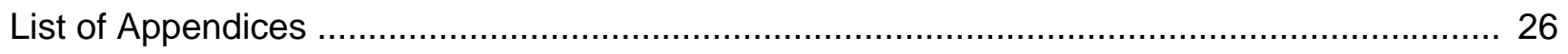




\section{List of Figures}

Figure 1. FT Calculation For a Given Energy Grid Interval ...................................... 8

Figure 2. Evolution of Energy Grid Structure for U-238 with Temperature ..................... 9

Figure 3. Doppler Reactivity Defect for UO2 Benchmark Cases ................................ 15

Figure 4. Doppler Reactivity Defect for PuO2 Benchmark Cases ................................ 15

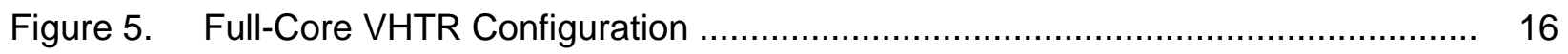

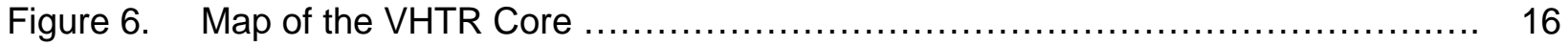

Figure 7. Axial temperature distributions for the VHTR fuel regions .................... 17

Figure 8. Axial temperature distributions for the VHTR reflector regions ........................ 17

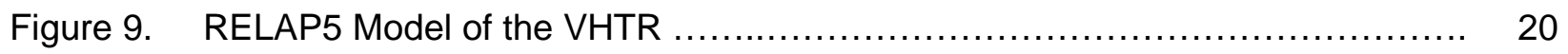

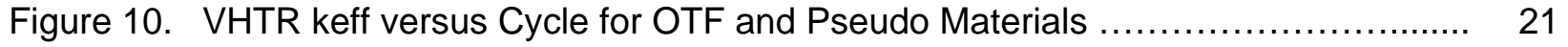

Figure 11. Axial Temperature Distribution for Middle Core Ring with Pseudo Materials ...... 21

Figure 12. Axial Temperature Distribution for Middle Core Ring with OTF...................... 22

Figure 13. Axial Temperature Distributions for Iterations 5-6 for Middle Core Ring.............. 22 


\section{List of Tables}

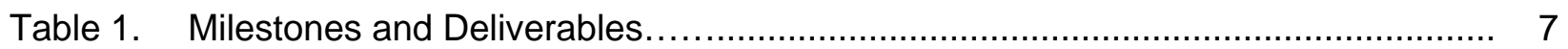

Table 2. Number of Union Energy Grid Points for U-238 Cross Sections....................... 9

Table 3. Comparison of OTF versus Standard Method for the UO2 Cases ................ 13

Table 4. Comparison of OTF versus Standard Method for the PuO2 Cases ................ 14

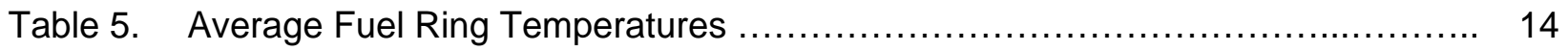

Table 6. Approximate Temperature Distribution for VHTR Test Problem ................. 18

Table 7. Comparison of Results using MCNP with OTF vs. Conventional Method .......... 19 


\begin{abstract}
A new method to obtain Doppler broadened cross sections has been implemented into MCNP, removing the need to generate cross sections for isotopes at problem temperatures. Previous work had established the scientific feasibility of obtaining Doppler-broadened cross sections "on-the-fly" (OTF) during the random walk of the neutron. Thus, when a neutron of energy $\mathrm{E}$ enters a material region that is at some temperature $T$, the cross sections for that material at the exact temperature $\mathrm{T}$ are immediately obtained by interpolation using a high order functional expansion for the temperature dependence of the Doppler-broadened cross section for that isotope at the neutron energy $\mathrm{E}$. A standalone Fortran code has been developed that generates the OTF library for any isotope that can be processed by NJOY. The OTF cross sections agree with the NJOY-based cross sections for all neutron energies and all temperatures in the range specified by the user, e.g., 250K - 3200K. The OTF methodology has been successfully implemented into the MCNP Monte Carlo code and has been tested on several test problems by comparing MCNP with conventional ACE cross sections versus MCNP with OTF cross sections. The test problems include the Doppler defect reactivity benchmark suite and two full-core VHTR configurations, including one with multiphysics coupling using RELAP5-3D/ATHENA for the thermal-hydraulic analysis. The comparison has been excellent, verifying that the OTF libraries can be used in place of the conventional ACE libraries generated at problem temperatures. In addition, it has been found that using OTF cross sections greatly reduces the complexity of the input for MCNP, especially for full-core temperature feedback calculations with many temperature regions. This results in an order of magnitude decrease in the number of input lines for full-core configurations, thus simplifying input preparation and reducing the potential for input errors. Finally, for full-core problems with multiphysics feedback, the memory required to store the cross section data is considerably reduced with OTF cross sections and the additional computational effort to use OTF cross sections is negligible.
\end{abstract}

This is a joint project with the University of Michigan, Argonne National Laboratory, and Los Alamos National Laboratory. 


\section{Introduction}

Doppler broadening of nuclear cross sections is a key nuclear phenomenon that occurs during nuclear reactor operation and has important implications for reactor safety. Resonance cross sections change significantly due to the relative motion between the incoming neutron and the target nucleus and must be accounted for in the analysis of reactors at operating temperature. If a continuous energy Monte Carlo code such as MCNP [1] is used for the neutronics portion of a multi-physics simulation with thermal-hydraulic feedback, cross section libraries must be generated for all isotopes over the range of temperatures expected in the reactor. In a light water reactor (LWR), this process can result in tens of thousands of material temperatures for which broadened cross sections need to be generated using NJOY [2]. Since a cross section file for a typical isotope at a specified temperature may be $10 \mathrm{MB}$ in size, this can result in a huge increase in fast memory to store the cross section files. Moreover, the need to assign different material IDs to cross section files for the same isotope at different temperatures greatly complicates (and expands) the input files for problems with temperature feedback.

Recent research by Yesilyurt et al. [3-5] has shown the feasibility of replacing the cross section files generated at specific temperatures with functional expansions that represent the detailed energy and temperature dependence of the cross sections. This allowed "on-the-fly" (OTF) determination of Doppler-broadened cross sections at the exact temperature encountered by the neutron during its random walk through the problem geometry.

The goal of this project was to take the OTF capability beyond the scientific feasibility stage reported in [3-5] and extend it to a production capability by incorporating it into the MCNP Monte Carlo code, providing reactor analysts the capability to analyze coupled neutronic/TH calculations without the need to generate cross section libraries at all temperatures that are anticipated during the simulation.

\section{Project Scope}

The development of a production-level OTF Doppler-broadening capability in MCNP involved a number of development efforts:

- Extension of the original OTF methodology, which was applied only to capture cross sections, to all cross section types that are broadened by NJOY.

- Replacement of the set of research codes used to generate OTF datasets with a single verified and validated Fortran code that can be distributed to the user community.

- Modifications to the MCNP code to allow use of OTF cross sections.

- Generation of the OTF libraries for a set of isotopes and a temperature range of practical interest to reactor analysts.

- Validation of the OTF capability by using MCNP to analyze test problems with both OTF and conventional cross section sets.

Table 1 lists the milestones and deliverables that were proposed to carry out the work described above. All of these milestones were completed successfully and the remainder of this report describes this work in detail. 


\begin{tabular}{|c|l|l|}
\hline Milestone & \multicolumn{1}{|c|}{ Description } & Result \\
\hline 1 & $\begin{array}{l}\text { Identification of isotopes to be treated by OTF Doppler } \\
\text { broadening methodology and incorporated into MCNP }\end{array}$ & Completed \\
\hline 2 & $\begin{array}{l}\text { Generation of functional expansions for extended set of } \\
\text { isotopes and reactions }\end{array}$ & Completed \\
\hline 3 & Modification of MCNP to allow OTF Doppler broadening & Completed \\
\hline 4 & $\begin{array}{l}\text { Completion of validation runs for modified MCNP with OTF } \\
\text { Doppler broadening }\end{array}$ & Completed \\
\hline 5 & Issue Final Report & This report \\
\hline
\end{tabular}

Table 1. Milestones and Deliverables

\section{OTF Methodology}

\section{A. Overall description}

The basic idea is to expand the temperature dependence of a particular cross section at a given neutron energy in a functional expansion in temperature for every nuclide and every cross section type that is subject to Doppler broadening. The expansion coefficients are a function of isotope, cross section type, and energy grid point $E_{g}$. For example, the capture cross section for a given isotope at a given energy grid point $E_{g}$ might have the following temperature expansion:

$$
\sigma_{\gamma}\left(\mathrm{T}, \mathrm{E}_{\mathrm{g}}\right) \cong \sum_{\mathrm{i}=1}^{\mathrm{N}} \frac{\mathrm{a}_{\mathrm{g}, \mathrm{i}}}{\mathrm{T}^{\mathrm{i} / 2}}+\sum_{\mathrm{i}=1}^{\mathrm{N}} \mathrm{b}_{\mathrm{g}, \mathrm{i}} \mathrm{T}^{\mathrm{i} / 2}+\mathrm{C}_{\mathrm{g}}
$$

The expansion coefficients $\mathrm{a}_{\mathrm{g}, \mathrm{i}}, \mathrm{b}_{\mathrm{g}, \mathrm{i}}$, and $\mathrm{c}_{\mathrm{g}}$ are determined by a temperature-dependent regression model based on the exact Doppler broadened cross sections [6] at that energy grid point. In essence, these coefficients allow the determination of cross sections for all neutron energies and all temperatures over an arbitrary temperature range (e.g. 250K - 3200K) determined by the analyst who generates the OTF library. We have found that $\mathrm{N}=8$ (corresponding to a 17 term expansion) yields agreement with NJOY-determined cross sections to within .1\% for all cross section types over the entire energy and temperature range specified for the OTF library.

The functional expansion allows Doppler broadened cross sections to be determined during the random walk of the neutrons - if a neutron with energy $E$ enters a material region that is at some temperature $\mathrm{T}$, the Doppler-broadened cross sections for that material are immediately determined "on-the-fly" (OTF) using linear interpolation of the cross sections determined by Eq. (1) at the energy grid points that bracket the neutron energy E. The OTF library replaces all cross sections that are Doppler broadened by NJOY but does not replace other cross sections such as $S(\alpha, \beta)$ cross sections, high threshold cross sections, cross sections in the unresolved range, or collision physics data such as outgoing energy-angle pairs. The key elements in the OTF methodology are described in the following sections. 


\section{B. Creating a union energy mesh for a nuclide}

The first step is to construct a union energy grid over a predefined temperature range of interest for each of the isotopes. Here, "union" refers to a common energy grid structure for a single nuclide that can be used for all of the broadened cross sections for that nuclide at all the predefined temperature points while maintaining satisfactory agreement with the exact cross section values. Appendix A describes the methodology to construct a union energy grid for $U$ 238, a particularly challenging isotope because of the strong energy dependence of the cross sections including sharp resonances for capture, fission, and scatter. A brief summary of the union grid methodology is given here and Appendix A should be consulted for the details.

The union energy grid is a common energy grid for the tabulation of all temperature-dependent cross sections for a given isotope, and it holds for the entire temperature range specified by the user. Thus U-238 will have all of its cross sections, including temperature-dependent cross sections over the specified temperature range (e.g., 250K - 3200K), tabulated on the same energy grid. The determination of the union energy grid is based on satisfying the NJOY fractional tolerance (FT) for all of these cross sections and temperatures. The FT is defined as the relative difference in cross sections between the values of exact and linearly interpolated cross sections at mid-points between successive energy grid points. Figure 1 depicts a typical energy grid interval with tabulated cross sections given at energies $E_{1}$ and $E_{2}$. Satisfying the FT means that the cross section at the mid-point energy, interpolated from the tabulated cross sections at $E_{1}$ and $E_{2}$, agrees with the exact cross section from NJOY to within the tolerance specified by FT. The value of FT has been chosen to be .1\% for this project.

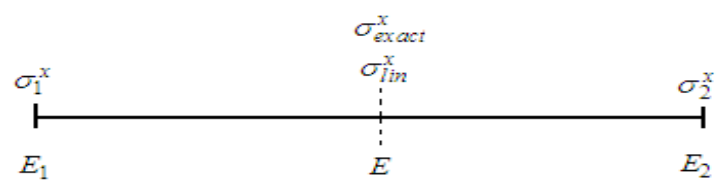

Figure 1. FT Calculation For a Given Energy Grid Interval

The energy grid spacing $\Delta \mathrm{E}=\mathrm{E}_{2}-\mathrm{E}_{1}$ satisfied the $\mathrm{FT}$ criterion if

$$
\frac{\left|\sigma_{\text {exact }}^{\mathrm{x}}-\sigma_{\text {lin }}^{\mathrm{x}}\right|}{\sigma_{\text {exact }}^{\mathrm{x}}}<\mathrm{FT}
$$

The user specifies a union temperature grid with specified spacing $\Delta T_{\text {union }}$ which is used for all cross sections and all isotopes in the OTF library. For example, if the union temperature grid spacing is $\Delta \mathrm{T}_{\text {union }}=50 \mathrm{~K}$ and the base cross section library is at $\mathrm{T}_{0}=250 \mathrm{~K}$, then the union temperature grid includes $250 \mathrm{~K}, 300 \mathrm{~K}, 350 \mathrm{~K}$, etc., to the maximum temperature specified, e.g., $3200 \mathrm{~K}$. The union temperature grid is only used to generate the union energy grid and is not used beyond that task.

Figure 2 illustrates why the union energy grid is important. When the temperature increases, the number of energy grid points required to satisfy a given FT decreases near the peak of a resonance as it smooths out, resulting in a coarser energy grid structure. However, when the temperature increases, more energy grid points are required for the middle and wings of a resonance to satisfy the same FT, yielding a finer energy grid structure. For an increase in temperature, the net result is a coarser energy grid. By design, energy grid points are only 
added to the union energy grid, and never taken away, so the overall effect is a modest increase in energy grid points with temperature as a result of the increased energy resolution needed for the middle and wings of the resonances.

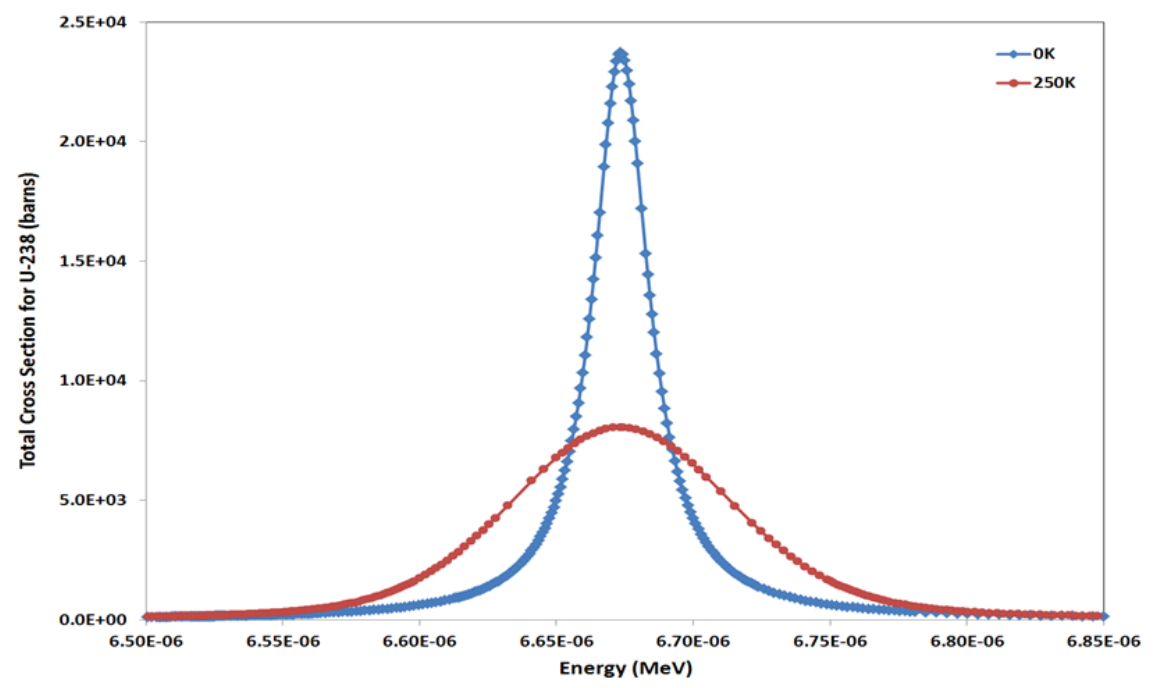

Figure 2. Evolution of Energy Grid Structure for U-238 with Temperature.

The initial union energy grid corresponds to the base set of cross sections that have been generated by NJOY at a specified temperature and satisfies Eq. (2) by construction. The construction of the union energy grid for a specific isotope then consists of a loop over the energy grid, with an inner loop over the union temperature points and cross section types for that isotope, halving the current energy grid spacing until Eq. (2) is satisfied for every temperature and cross section type. The end result is an energy grid that is unique for each isotope and satisfies Eq. (2) for all temperatures and cross section types.

For example, Table 2 shows the extra grid points that were added to the starting energy grid for U-238 at 250K, in order to satisfy Eq. (2) for capture, elastic scattering, fission, and total cross sections, in that order. The base set at $250 \mathrm{~K}$ had 134,437 energy points and the resultant union energy grid had an additional 10,866 energy points.

Table 2. Number of Union Energy Grid Points for U-238 Cross Sections

\begin{tabular}{|l|l|c|}
\hline Reaction type & Number & Increase \\
\hline (Base set) & 134,437 & - \\
\hline Capture & 138,080 & 3643 \\
\hline Scattering & 143,216 & 5136 \\
\hline Fission & 143,574 & 358 \\
\hline Total & 145,303 & 1729 \\
\hline (Overall) & 145,303 & 10,866 \\
\hline
\end{tabular}




\section{Determining Doppler-broadened cross sections on the union energy grid}

The Doppler-broadened cross sections are determined at every energy point in the union energy grid using NJOY-derived routines that implement Cullen's exact Doppler broadening equation [6]. These cross sections are determined for a set of temperatures, called the fit temperature grid, using a fit temperature interval $\Delta \mathrm{T}_{\text {fit }}$ over the specified temperature range.

The fit temperature grid is separate from the union temperature grid and while the two grids can be the same, in practice the fit temperature grid is usually much finer than the union grid. Exact Doppler-broadened cross sections are tabulated on the fit temperature grid for each energy on the union energy grid. We have used $\Delta \mathrm{T}_{\text {union }}=100 \mathrm{~K}$ and $\Delta \mathrm{T}_{\text {fit }}=10 \mathrm{~K}$ for the OTF cross section sets that were used in this report for the benchmark calculations discussed below.

\section{Calculation of OTF expansion coefficients}

This basic methodology described in [3-5] has been consolidated in a single standalone code fit_otf, which can be run in either of two modes, one for generating union energy grids and one for generating the expansion coefficients. The computation mode is controlled through the specification of input options which also define the isotope in question and provide the parameters for generating the union energy grid and the union and fit temperature grids.

In both grid-generation mode and coefficient fitting mode, fit_otf reads an initial, user-specified ACE input file for use in determining which cross sections must be fit. Options are available to use either ACE files supplied in the general MCNP distribution or ACE files generated by the user. In both modes, the program uses Cullen's method [6] to generate exact Dopplerbroadened cross sections at various temperatures. Note that the temperature of the ACE file must be equal to or lower than the lowest temperature required for the fit. For generating union energy grids, the user must also specify the minimum and maximum energies for the fit, the minimum and maximum temperatures, and the union temperature increment $\Delta T_{\text {union }}$ at which to examine the exact cross sections to determine whether additional energy points are needed to assure linear interpolation to within $0.1 \%$. The algorithm for determining the union grid essentially follows the steps outlined in Section IV.B. The general algorithm and pertinent implementation details of the fit_otf methodology for computing expansion coefficients for a given isotope are described below.

Treating the expansion coefficients in Eq. (1) as the unknown variables, the expansion for a given cross section type at a given energy is evaluated for all temperatures on the fit temperature grid, yielding a system of linear equations $\boldsymbol{A x}=\boldsymbol{b}$, in which the vector $\boldsymbol{b}$ contains the cross sections at each temperature, $\boldsymbol{x}$ is the vector of the coefficients, and each row of the matrix $\boldsymbol{A}$ holds the values of the temperature corresponding to the elements in the given row raised to the powers of the expansion. Such a system typically lends itself to easy solution by a least squares minimization approach. Unfortunately, because the temperature range in OTF problems can be rather large and the expansion has both positive and negative powers of the temperature, the matrix $\boldsymbol{A}$ usually has a poor condition number, so solving the system generally requires singular value decomposition (SVD). In the current work, the routine MINFIT from the SLATEC (EISPACK) library [7] has been adapted for FORTRAN 90 and implemented in fit_otf. Initially it was found that quadruple precision arithmetic was required to produce reliable fits, but it was later determined that double precision calculations would suffice if the OTF expansion was made not in terms of the temperature $T$ but rather in terms of a scaled temperature given by $\left(T-T_{1}-T_{\text {off }}\right) /\left(T_{2}-T_{1}-T_{\text {off }}\right)$. In this scaling, the offset temperature $T_{\text {off }}=\left(T_{1}-T_{2}\right) / 50$ and $T_{1}$ and $T_{2}$ are the upper and lower temperatures of the fit temperature grid. 
Since the variation with temperature is significantly different at different energies for the same cross section and is also different at the same energy for different cross sections, the number of coefficients required for accurate modeling can vary greatly with both energy and cross section type. Using a fixed expansion order would result in an extremely large number of unnecessary coefficients, and so to minimize the size of the OTF data sets, the number of coefficients of the expansion is allowed to vary with both energy and cross section type.

The generation of the coefficients proceeds as follows. At a given energy in the union energy grid, exact cross section values are generated at all temperatures on the fit temperature grid for all cross sections types being fit. Starting with expansion order 1 (meaning 3 expansion coefficients), the temperature matrix $\boldsymbol{A}$ is then calculated and MINFIT is called to generate for each cross section type the least squares coefficients for the given fit-order. (Note that since $\boldsymbol{A}$ depends only on the temperatures of the fit grid and the order of the expansion coefficients, it is independent of cross section type and so the same, computationally expensive SVD decomposition can be applied to all cross section types at once, rather than having to be performed independently.) Next, cross section values are calculated at each temperature in the grid using the fit coefficients and compared to the exact value for each cross section type. If any of the fitted values differ from the exact values by more than the input tolerance (the default tolerance is $0.1 \%$ relative difference, similar to the fractional tolerance in NJOY), the process is repeated using a higher order fit (but only for those cross section types not already shown to be accurately fit with a lower order expansion). Once all of the cross section types are fit to better than $0.1 \%$, the loop over expansion order is broken and the program moves to the next union grid energy.

The fit_otf program utilizes multicore parallel threading to reduce computer run times. Note that this code does not replace NJOY, but supplements it, providing a convenient mechanism for extending the Doppler broadening to a wide range of temperatures.

\section{E. Sensitivity study for $\Delta T_{\text {union }}$ and $\Delta T_{\text {fit }}$}

A sensitivity study on different choices of $\Delta T_{\text {union }}$ and $\Delta T_{\text {fit }}$ deltaT(fit) and deltaT(union) for U-238 was carried out by Yesilyurt and is attached as Appendix A. A brief summary is given here. It was concluded that $\Delta \mathrm{T}_{\text {union }}=50 \mathrm{~K}$ was sufficient to mainain a fractional tolerance of $.1 \%$ with a reasonable number of energy grid points over the temperature range $250 \mathrm{~K}-3200 \mathrm{~K}$. Also, the OTF cross sections at $1975 \mathrm{~K}$, midway between the union temperature points $1950 \mathrm{~K}$ and $2000 \mathrm{~K}$, agreed with NJOY to within $.00001 \%$. A sensitivity study on $\Delta \mathrm{T}_{\text {fit }}$ was also carried out and it was concluded that $\Delta \mathrm{T}_{\text {fit }}=1 \mathrm{~K}$ was a very conservative choice for fitting the expansion coefficients (with 17 terms) but $25 \mathrm{~K}$ was too large, yielding a few cross sections outside the $.1 \%$ fractional tolerance. It was decided to use $\Delta \mathrm{T}_{\text {fit }}=10 \mathrm{~K}$ to obtain acceptable results with reasonable computational efficiency.

\section{F. Construction of the OTF library}

The above steps have been integrated into the fit_otf FORTRAN 90 code. The actual generation of the OTF library is dependent on a number of quantities that are user-specified:

- the list of nuclides,

- the base cross section library for each nuclide,

- the temperature range over which the OTF cross sections are generated,

- the error criterion corresponding to FT, 
- the temperature intervals $\Delta \mathrm{T}_{\text {union }}$ and $\Delta \mathrm{T}_{\text {fit }}$, and

- the maximum order of expansion to be used for the high precision fit.

Another important consideration is the cross section types to be included in the OTF library. This decision is determined by whatever NJOY does: if a cross section type is broadened by NJOY, it is included in the OTF library. The fit_otf code reads in the NJOY base library and identifies which of the cross section types (MT) has Doppler-broadened cross sections. The energy grid, over which these cross sections are broadened, ranges from $.00001 \mathrm{MeV}$ to the highest energy less than the unresolved energy range or the energy at which high threshold reactions occur, whichever is lowest. The base cross section set contains all of the temperature independent cross section information, such as the unresolved cross sections and collision physics data, for each isotope in the OTF library.

\section{G. Implementation of OTF methodology in MCNP}

The previous sections describe the methodology used to generate the OTF library. The use of the OTF library in MCNP requires some changes to MCNP, which is based on having separate cross section files for every temperature rather than one file for all temperatures with the OTF library. The number of modifications was modest, involving only a few routines that retrieve and interpolate cross section data as a function of neutron energy. Since there is only one OTF cross section set (actually a set of expansion coefficients) per isotope regardless of the temperature range, the TMP card simply designate the cell temperature, and if the isotope is in that cell, the OTF expansion for that isotope is evaluated at that temperature. The use of TMP illustrates the substantial reduction in input complexity with OTF - a material (isotope) has the same ID in every cell in which it is found, regardless of the temperature of the cell. This is to be contrasted to the conventional methodology where a separate material ID has to be assigned to an isotope if it is in a cell at a different temperature. This results in a proliferation of material IDs and associated cross section files for full-core simulations with TH feedback, increasing the size of the cross section files as well as increasing the complexity of the input file.

\section{Assessment of OTF Methodology}

\section{A. Test problems}

Four test problems have been analyzed with MCNP with both OTF cross section files and standard NJOY-based cross section files, allowing direct code-to-code comparisons on realistic reactor configurations that are encountered by analysts. The primary goal of these comparisons is to verify the accuracy of the OTF methodology and its correct implementation in MCNP. These comparisons also allow an assessment of the computational efficiency of using OTF in MCNP and a demonstration of the substantial simplification of the MCNP input process and resultant reduction in the size of the input files for temperature-dependent problems. The four test problems are listed below:

- Doppler reactivity benchmark suite

- Doppler reactivity benchmark suite with radial temperature dependence

- Full core VHTR with imposed temperature distribution

- Full core VHTR with thermal-hydraulic coupling

The following sections discuss each of these test problems in detail. 


\section{B. Doppler reactivity benchmark suite}

The Doppler reactivity benchmark suite [8] was chosen for the first comparison of the OTF methodology versus the conventional approach using ACE cross sections generated at the specific temperatures. This problem, named Doppler-1 for this report, compares keff for HZP (hot, zero power) and HFP (hot, full power) conditions for a fuel pin cell typical of a PWR. The basic model for this benchmark is a PWR fuel pin cell with three regions - fuel, clad, and moderator. For the HZP cases, all regions are at 600K. For the HFP cases, the fuel region is at $900 \mathrm{~K}$ while the clad and moderator regions are unchanged at $600 \mathrm{~K}$. There are three different fuel regions: UO2, MOX with reactor-grade plutonium, and MOX with weapons-grade plutonium. There is a range of uranium enrichments for the UO2 cases and a range of PuO2 weight percent for the MOX cases, and there are different plutonium isotopic vectors for the reactorgrade and weapons-grade MOX cases. This benchmark is strictly a computational benchmark with no experimental results and is used internationally for comparing codes.

Simulations were performed using 10000 cycles (9500 active) of 10000 histories for each benchmark case. Tables 3 and 4 presents comparisons of MCNP results using OTF cross sections and conventional ACE cross sections. Table 3 presents the UO2 cases and Table 4 presents the reactor-grade MOX cases. (The weapons-grade MOX cases were not analyzed.)

The results in Tables 3 and 4 clearly indicate that the OTF methodology is working correctly, providing results that agree with standard NJOY-MCNP calculations within statistics.

Table 3. Comparison of OTF versus Standard Method for the UO2 Cases

\begin{tabular}{|c|c|c|c|c|c|}
\hline \multirow[t]{2}{*}{ Enrichment } & \multirow[t]{2}{*}{ Method } & \multicolumn{2}{|c|}{ keff (one $\sigma$ ) } & \multicolumn{2}{|c|}{$\begin{array}{l}\text { Doppler Defect (one } \sigma \text { ) } \\
(\mathrm{pcm} / \mathrm{K})\end{array}$} \\
\hline & & HZP & HFP & MCNP & Avg from [8] \\
\hline \multirow{2}{*}{$.711 \%$} & OTF & $0.66564(04)$ & $0.65973(04)$ & $-4.486(43)$ & -4.27 \\
\hline & Std & $0.66565(04)$ & $0.65975(04)$ & $-4.478(43)$ & -4.27 \\
\hline \multirow{2}{*}{$1.6 \%$} & OTF & $0.96084(06)$ & $0.95273(06)$ & $-2.953(31)$ & -3.00 \\
\hline & Std & $0.96091(06)$ & $0.95261(06)$ & $-3.022(31)$ & -3.00 \\
\hline \multirow{2}{*}{$2.4 \%$} & OTF & $1.09914(06)$ & $1.08991(06)$ & $-2.568(24)$ & -2.52 \\
\hline & Std & $1.09904(06)$ & $1.08996(06)$ & $-2.527(24)$ & -2.52 \\
\hline \multirow{2}{*}{$3.1 \%$} & OTF & $1.17713(06)$ & $1.16751(06)$ & $-2.333(21)$ & -2.30 \\
\hline & Std & $1.17719(06)$ & $1.16749(06)$ & $-2.353(21)$ & -2.30 \\
\hline \multirow{2}{*}{$3.9 \%$} & OTF & $1.23974(06)$ & $1.22975(06)$ & $-2.184(19)$ & -2.20 \\
\hline & Std & $1.23973(07)$ & $1.22966(07)$ & $-2.202(22)$ & -2.20 \\
\hline \multirow{2}{*}{$4.5 \%$} & OTF & $1.27516(06)$ & $1.26510(06)$ & $-2.079(18)$ & -2.18 \\
\hline & Std & $1.27514(07)$ & $1.26503(07)$ & $-2.089(20)$ & -2.18 \\
\hline \multirow{2}{*}{$5.0 \%$} & OTF & $1.29900(07)$ & $1.28933(07)$ & $-1.925(20)$ & -2.06 \\
\hline & Std & $1.29897(07)$ & $1.28924(06)$ & $-1.937(18)$ & -2.06 \\
\hline
\end{tabular}

For these simple pin cell runs, using MCNP with OTF cross sections yielded a performance penalty of $15-20 \%$ versus using conventional ACE cross sections. When more complicated 
problems are analyzed, such as the full-core VHTR, this performance penalty goes away. For this modest number of isotopes and temperatures, the memory required to store cross sections was slightly larger with OTF than with ACE cross sections. However, the same OTF library can be used for the radial temperature dependent case that follows, which is not the case with ACE cross sections.

\section{Doppler reactivity benchmark suite with radial temperature dependence}

To demonstrate the functionality and capability of the OTF methodology, the Doppler-1 test problem was modified (named Doppler-2) with the fuel subdivided into 10 concentric, equally spaced regions over which the temperature was allowed to vary. If the temperature at the edge of the fuel is $600 \mathrm{~K}$ and the average temperature is $900 \mathrm{~K}$, it can be shown that the radial temperature distribution for a constant power density is given by $T(r)=1200-600(r / R)^{2}$, where $\mathrm{R}$ is the radius of the fuel. With this temperature distribution in the fuel, the average fuel temperatures in the 10 rings are given in Table 5 , where ring $\# 1$ is on the centerline of the fuel pin.

Table 4. Comparison of OTF versus Standard Method for the PuO2 Cases

\begin{tabular}{|c|c|c|c|c|c|}
\hline \multirow{2}{*}{ Enrichment } & \multirow{2}{*}{ Method } & \multicolumn{2}{|c|}{ keff (one $\sigma)$} & \multicolumn{2}{c|}{$\begin{array}{c}\text { Doppler Defect (one } \sigma \text { ) } \\
\text { (pcm/K) }\end{array}$} \\
\cline { 3 - 6 } & & HZP & HFP & MCNP & Avg from [8] \\
\hline \multirow{3}{*}{$\mathbf{0 . 0 \%}$} & OTF & $0.66564(04)$ & $0.65973(04)$ & $-4.486(43)$ & -4.27 \\
\cline { 2 - 6 } & Std & $0.66565(04)$ & $0.65975(04)$ & $-4.478(43)$ & -4.27 \\
\hline \multirow{3}{*}{$\mathbf{1 . 0 \%}$} & OTF & $0.94493(06)$ & $0.93520(06)$ & $-3.670(32)$ & -3.72 \\
\cline { 2 - 6 } & Std & $0.94483(06)$ & $0.93515(06)$ & $-3.652(32)$ & -3.72 \\
\hline \multirow{2}{*}{$\mathbf{2 . 0 \%}$} & OTF & $1.02069(07)$ & $1.00973(07)$ & $-3.545(32)$ & -3.60 \\
\cline { 2 - 6 } & Std & $1.02071(07)$ & $1.00969(07)$ & $-3.564(32)$ & -3.60 \\
\hline \multirow{3}{*}{$\mathbf{4 . 0 \%}$} & OTF & $1.07588(07)$ & $1.06410(07)$ & $-3.430(29)$ & -3.50 \\
\cline { 2 - 6 } & Std & $1.07577(07)$ & $1.06414(07)$ & $-3.386(29)$ & -3.50 \\
\hline \multirow{2}{*}{$\mathbf{6 . 0 \%}$} & OTF & $1.10457(07)$ & $1.09271(07)$ & $-3.275(27)$ & -3.30 \\
\cline { 2 - 6 } & Std & $1.10451(07)$ & $1.09280(07)$ & $-3.234(27)$ & -3.30 \\
\hline \multirow{2}{*}{$\mathbf{8 . 0 \%}$} & OTF & $1.12790(07)$ & $1.11606(07)$ & $-3.135(26)$ & -3.20 \\
\cline { 2 - 6 } & Std & $1.12793(07)$ & $1.11607(07)$ & $-3.140(26)$ & -3.20 \\
\hline
\end{tabular}

The assumption was made in moving from a single fuel region to 10 regions that the atomic number densities in the fuel did not vary with temperature and were equal to the $900 \mathrm{~K}$ densities.

Table 5. Average Fuel Ring Temperatures

\begin{tabular}{|l|c|c|c|c|c|c|c|c|c|c|}
\hline Ring \# & $\mathbf{1}$ & $\mathbf{2}$ & $\mathbf{3}$ & $\mathbf{4}$ & $\mathbf{5}$ & $\mathbf{6}$ & $\mathbf{7}$ & $\mathbf{8}$ & $\mathbf{9}$ & $\mathbf{1 0}$ \\
\hline Temp (K) & 1197 & 1185 & 1160 & 1125 & 1077 & 1016 & 944 & 861 & 764 & 656 \\
\hline
\end{tabular}

This simulation was quite simple to perform with the OTF cross sections. Only the temperatures had to be specified for the ten fuel regions - unique material IDs for the different temperature zones were not needed. Since this was only a pin cell, the reduction in input cards is not 
particularly large ( $\sim 50$ cards), but if one were to analyze an assembly with T-H coupling, the reduction in the number of material IDs, hence input cards, might be in the thousands, and there would be a corresponding increase in the number of cross section files. Perhaps more important is reducing the potential for making input errors due to the decrease in the input complexity.

It is interesting to see the impact of the radial variation in temperature on the Doppler coefficient. Figure 3 shows the Doppler coefficients for the UO2 problems as a function of enrichment for the Doppler-1 cases versus the Doppler-2 cases, along with the benchmark results [8]. Figure 4 is a similar plot for the $\mathrm{PuO} 2$ problems. For all cases, the effect of the radially-dependent temperatures is to reduce the magnitude of the Doppler coefficient by nearly a factor of two. This phenomenon is well-known but has been difficult to assess with Monte Carlo due to the difficulty of dealing with standard cross section files. If one were to analyze the Doppler-2 test problem with conventional ACE cross sections, the number of MCNP input cards would have increased by a factor of 13 over the number required with OTF cross sections. Since Doppler-2 is a single pin cell, albeit with 10 radial regions, the input complexity for a full core analysis (or even an assembly) with detailed temperature dependence within each pin would be prohibitive with conventional cross sections but would be straightforward with OTF cross sections.

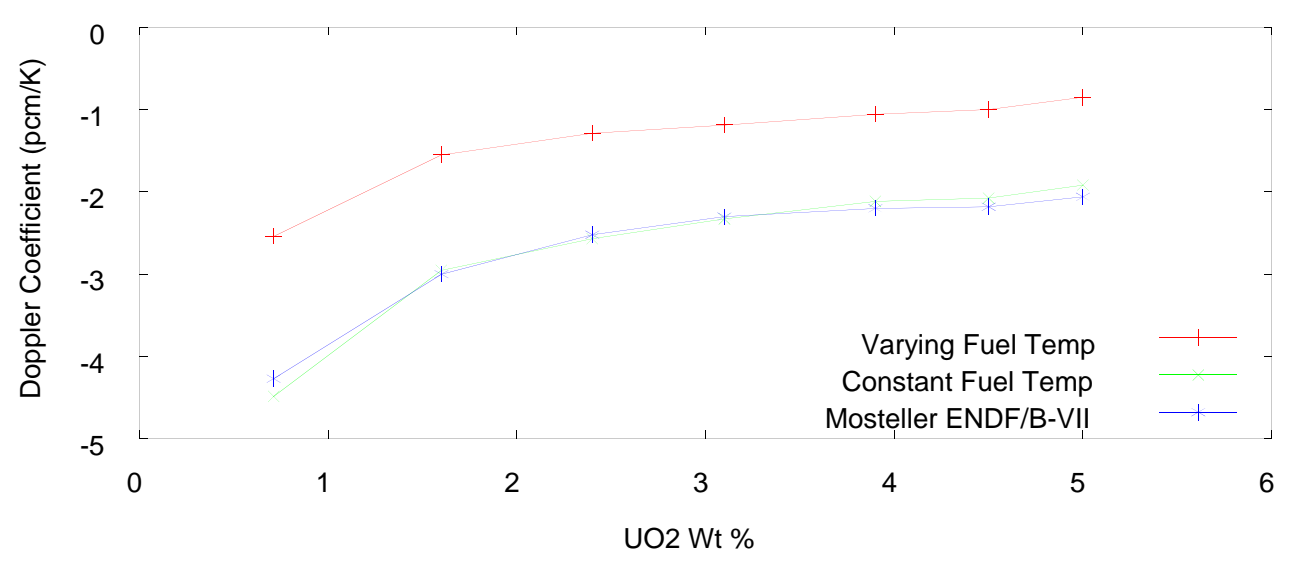

Figure 3. Doppler Reactivity Defect for UO2 Benchmark Cases

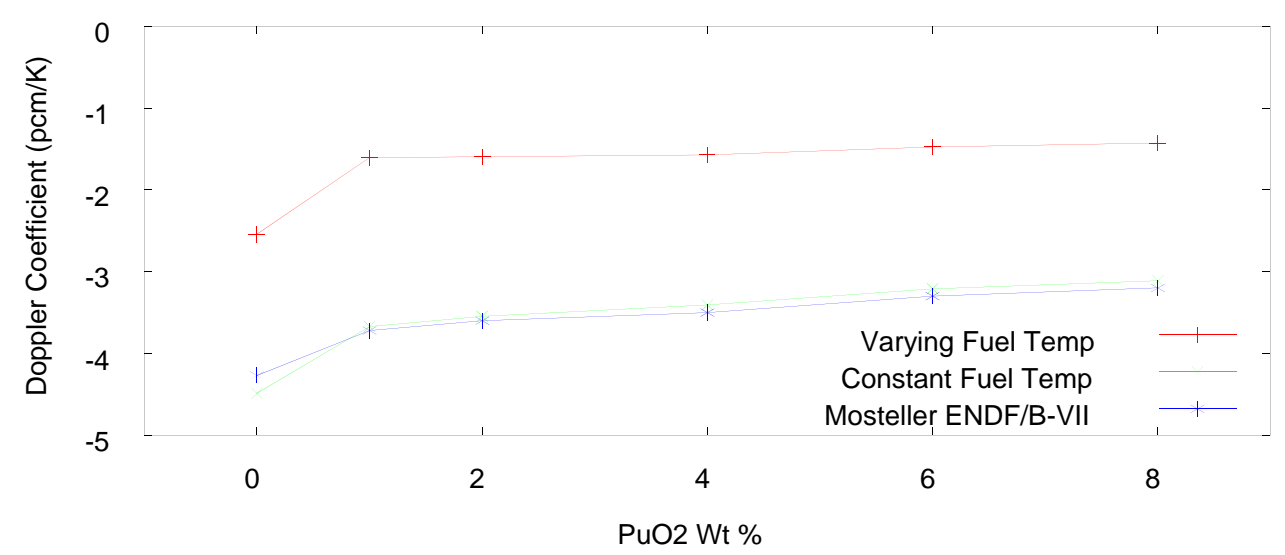

Figure 4. Doppler Reactivity Defect for PuO2 Benchmark Cases 


\section{Full core VHTR with imposed temperature distribution}

This test problem, named VHTR-1, is based on the full-core Very High Temperature Reactor (VHTR) with an imposed temperature distribution. It was chosen for the full-core assessment of the OTF methodology. The imposed temperature distribution was based on an earlier analysis [9] of the VHTR performed by the University of Michigan, which was a coupled neutronic/thermal-hydraulic (NTH) simulation of the VHTR using MCNP for the neutronic analysis and RELAP5-3D/ATHENA [10] for the thermal-hydraulic (TH) analysis. The goal of this earlier analysis was to determine the beginning-of-cycle (BOC) power and temperature distributions for the VHTR core at the rated power of $600 \mathrm{MWt}$, using both homogeneous and heterogeneous (resolved TRISO fuel particles) fuel. Figure 5 depicts the VHTR configuration analyzed in this earlier study.

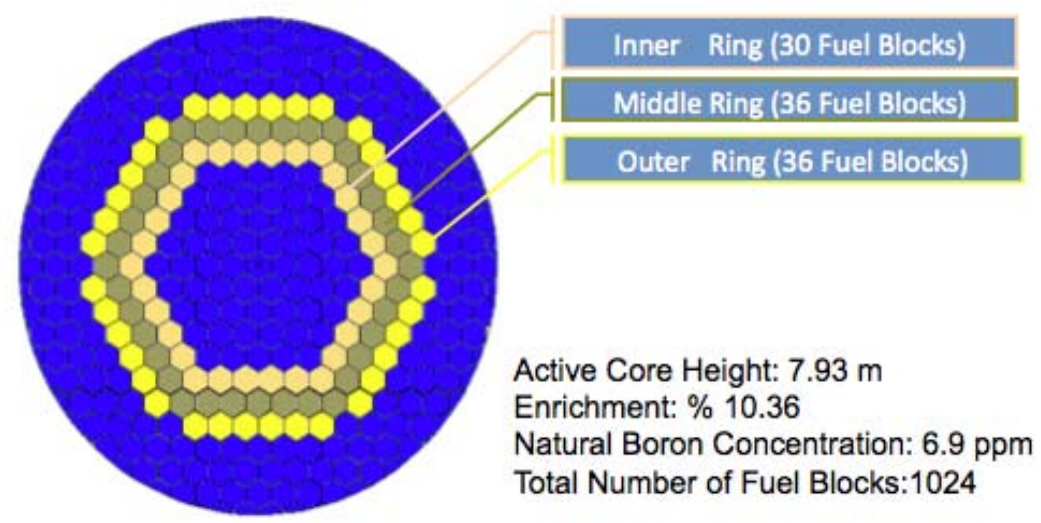

Figure 5. Full-Core VHTR Configuration

Figure 6 shows the temperature-dependent regions that are used. There are five axial fuel regions and upper and lower reflector regions. The five regions were divided into 10 axial zones, with additional reflector zones at the top and bottom.

\begin{tabular}{|c|c|c|c|c|}
\hline Iref & Iring & Mring & Oring & Oref \\
\hline 1 & 1 & 1 & 1 & 1 \\
\hline 2 & 2 & 2 & 2 & 2 \\
\hline 3 & 3 & 3 & 3 & 3 \\
\hline 4 & 4 & 4 & 4 & 4 \\
\hline 5 & 5 & 5 & 5 & 5 \\
\hline 6 & 6 & 6 & 6 & 6 \\
\hline 7 & 7 & 7 & 7 & 7 \\
\hline 8 & 8 & 8 & 8 & 8 \\
\hline 9 & 9 & 9 & 9 & 9 \\
\hline 10 & 10 & 10 & 10 & 10 \\
\hline 11 & 11 & 11 & 11 & 11 \\
\hline 12 & 12 & 12 & 12 & 12 \\
\hline
\end{tabular}

\section{Legend}

Core Inlet Plenum

12 Core Outlet Plenum

Iref Inner Reflector

Iring Inner Ring of Core

Mring Middle Ring of Core

Oring Outer Ring of Core

Oref Outer Reflector

Figure 6. Map of the VHTR Core 
Figures 7 and 8 show the axial temperature distributions in the fuel and reflector regions, respectively, that were used to estimate the imposed temperature distribution for the OTF test problem. The temperature distributions from the homogeneous fuel case were used since the purpose of the OTF test problems is to assess the accuracy and functionality of the OTF methodology compared to the standard approach, not the fidelity of the simulation.

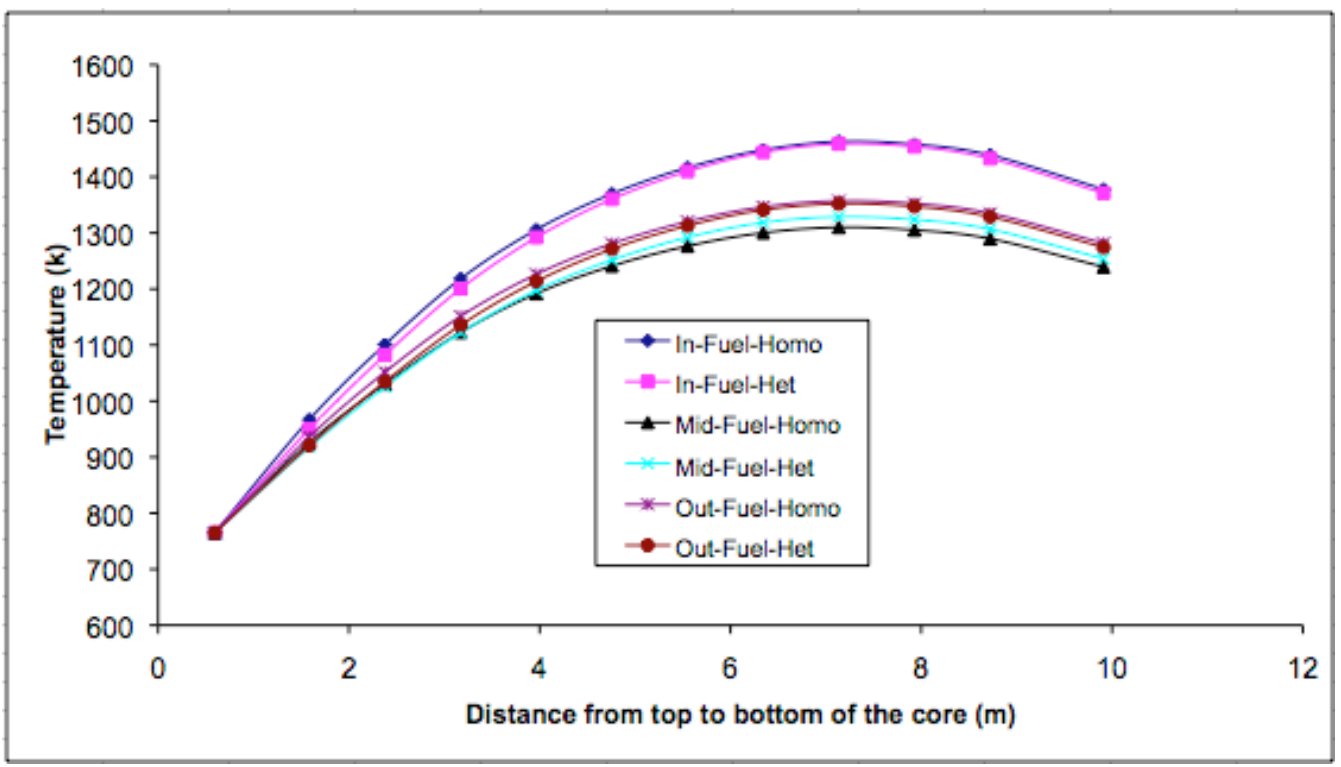

Figure 7. Axial temperature distributions for the VHTR fuel regions

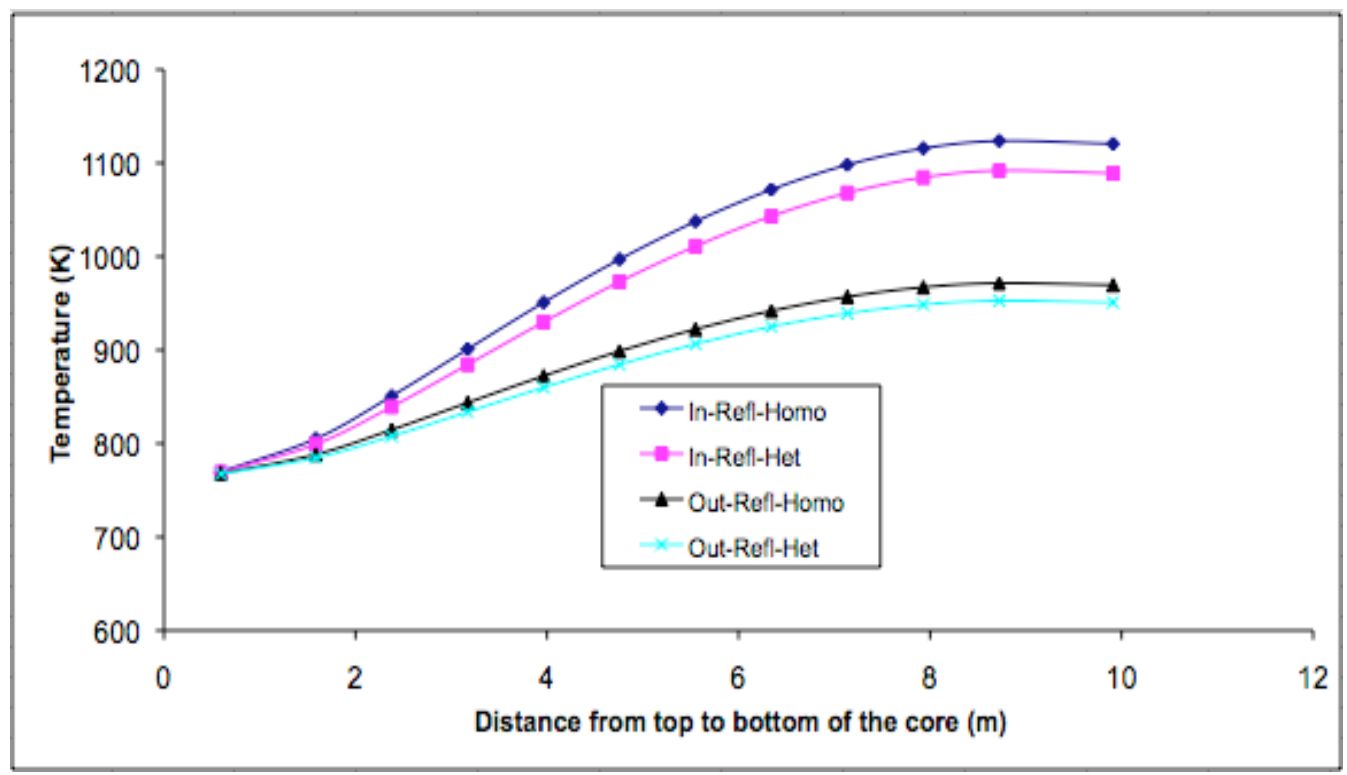

Figure 8. Axial temperature distributions for the VHTR reflector regions 
The analysis in [9] was performed with an approximate model, the pseudo-material model [11], to account for temperature-dependent cross sections. As a result, the results in [9] are not suitable as a reference case for comparison with the OTF method because the use of the approximate pseudo-material model introduces an unknown truncation error into the simulation. To remove this truncation error, it is necessary to use conventional ACE cross section libraries that are generated at the exact region temperatures. However, this would be a tedious effort because even though this is a modest problem with 60 temperature-dependent regions, the generation of ACE libraries for all the isotopes in the 60 regions could require as many as 300 NJOY runs, since there are three isotopes in each of the 30 reflector regions and 7 isotopes in each of the 30 fuel regions. (This observation by itself says a lot regarding the inherent difficulty in performing temperature-dependent Monte Carlo analyses with standard ACE cross section files because this is a simple full-core configuration with a small number of temperaturedependent regions.)

To avoid generating an inordinate number of ACE files, the VHTR-1 test problem was simplified by averaging the temperature distributions from Figures 7 and 8 over larger regions and assuming the inner and outer reflectors were at uniform temperatures. The resultant temperature distribution in degrees $\mathrm{K}$ is given in Table 6 , where the regions correspond to the VHTR map shown in Figure 6. While these assumptions are questionable from the standpoint of the fidelity of the simulation, the goal is to compare OTF with the standard model, not yield an accurate simulation, and these assumptions served that purpose.

This simplified temperature distribution still required generation of 52 ACE files, taking into account similar temperatures in different regions for the same isotopes. This is to be compared with 7 cross section files needed for the OTF method, one for each isotope regardless of which region it was in or what temperature it was at.

Table 6. Approximate Temperature Distribution for VHTR-1 Test Problem

\begin{tabular}{|c|c|c|c|c|c|}
\hline $\begin{array}{c}\text { Axial } \\
\#\end{array}$ & Iref & Iring & Mring & Oring & Oref \\
\hline 1 & 900 & 900 & 900 & 900 & 900 \\
\hline 2 & 900 & 900 & 900 & 900 & 900 \\
\hline 3 & 900 & 1100 & 1100 & 1100 & 900 \\
\hline 4 & 900 & 1100 & 1100 & 1100 & 900 \\
\hline 5 & 900 & 1100 & 1100 & 1100 & 900 \\
\hline 6 & 1100 & 1350 & 1250 & 1300 & 1100 \\
\hline 7 & 1100 & 1350 & 1250 & 1300 & 1100 \\
\hline 8 & 1100 & 1350 & 1250 & 1300 & 1100 \\
\hline 9 & 1100 & 1450 & 1300 & 150 & 1100 \\
\hline 10 & 1100 & 1450 & 1300 & 1350 & 1100 \\
\hline 11 & 1100 & 1400 & 1250 & 1250 & 1100 \\
\hline 12 & 1100 & 1400 & 1250 & 1250 & 1100 \\
\hline
\end{tabular}

The results of three MCNP simulations are shown in Table 7. The first two cases used the same number of histories and only differed in the use of OTF versus conventional ACE data files. The observed difference in keff for these two cases is a little larger than the sum of the two statistical uncertainties, so a second OTF run was performed, doubling the number of particles per cycle. 
Here is clear that the difference in eigenvalue is negligible, well within the uncertainty bands of the two simulations.

The results indicate that OTF yields essentially the same results as the conventional approach using separate ACE files for each temperature encountered in the problem. Moreover, the OTF approach is far simpler from the data preparation standpoint (only one OTF file per isotope needs to be generated) and from the MCNP input file standpoint (no separate material IDs for isotopes at different temperatures). The VHTR test problem was a realistic test problem to test the OTF methodology, since it is a full-core problem with an imposed temperature distribution taken from a converged NTH calculation of the VHTR.

Table 7. Comparison of Results using MCNP with OTF vs. Conventional Cross Sections

\begin{tabular}{|c|c|c|c|c|c|}
\hline Case & $\begin{array}{c}\text { \#Active } \\
\text { cycles }\end{array}$ & $\begin{array}{c}\text { Particles } \\
\text { per } \\
\text { cycle }\end{array}$ & keff & $\sigma$ & $\Delta$ keff \\
\hline Conventional & 400 & 100,000 & 1.15588 & .00014 & -- \\
\hline OTF & 400 & 100,000 & 1.15632 & .00013 & .00042 \\
\hline OTF & 400 & 200,000 & 1.15598 & .00009 & .00010 \\
\hline
\end{tabular}

\section{E. Full core VHTR with thermal-hydraulic coupling}

This test problem, named VHTR-2, is identical to the VHTR-1 test problem in the previous section except the VHTR temperature distribution is determined via nuclear/thermal-hydraulic (NTH) coupling with RELAP5-3D/ATHENA (RELAP5) rather than being imposed. The previous VHTR test problem allowed (with some simplifying assumptions) the use of ACE cross section that were generated at the exact temperatures of the regions, allowing an unambiguous comparison of MCNP with OTF versus conventional ACE cross sections. However, the generation of ACE files for the coupled problem is no longer practical because the temperatures in the 60 regions are not known a priori and it would take $300 \mathrm{NJOY}$ runs during each NTH iteration to generate the ACE libraries at all the temperatures that may be encountered in the 60 regions. For that reason, we decided to use the pseudo materials method [11], as was done in [9], to account for temperature feedback within MCNP. As a result, the comparison between the OTF and pseudo materials results is not as meaningful as in the VHTR-1 test problem. While the VHTR-2 test problem is not a perfect validation of the OTF methodology, it does demonstrate the significant advantage in ease of use of the OTF method compared to the conventional ACE file approach.

Accordingly, ENDF/B-VII library files for MCNP were prepared using NJOY99 (update 364) for isotopes B-10, B-11, and carbon at every $100 \mathrm{~K}$ from $700 \mathrm{~K}$ to $2400 \mathrm{~K}$. For isotopes U-235, U238, O-16, and Si-28, temperature-dependent library files were generated at every $100 \mathrm{~K}$ from $900 \mathrm{~K}$ to $2400 \mathrm{~K}$, giving a total of $125 \mathrm{MCNP}$ library files and $1.6 \mathrm{~GB}$ of data. For the lighter elements, generation of the data files took just several minutes, but, because of the need to generate probability tables for determining cross sections in the unresolved resonance region for the heavier materials, several hours of computation time on quad-core processors were required to prepare data for each heavy isotope.

Data files were also prepared for OTF cases in the temperature range $600 \mathrm{~K}$ to $3000 \mathrm{~K}$ based on standard MCNP libraries. Again, data preparation computation times were minimal for low $Z$ 
materials, increasing to approximately 40 minutes for $U-235$ and two hours for $U-238$, because of the large number of energy grid points. The total size of the OTF libraries was $359 \mathrm{MB}$.

Temperatures for moderator $S(\alpha, \beta)$ data were fixed at $1200 \mathrm{~K}$ in the fuel, and $1000 \mathrm{~K}$ in the reflectors. Because of the temperature variation in each region, each cell in the pseudo material implementation was a unique MCNP input material, requiring a total of 668 cards. In contrast, an OTF input file required 16 cards to specify the materials.

MCNP runs used 200,000 histories per cycle and 600 cycles, discarding the first 200, and required roughly 5 hours on 16 cores at the University of Michigan (UM) cluster.

MCNP output files were transferred from the CAC cluster to a Windows PC running Cygwin Linux. Axial power fractions were extracted from MCNP output using Perl [12] scripts modified from earlier work. Fractional power data was merged with an input template to produce input files which were then passed to RELAP5 to compute fuel-block average temperatures. In order to preclude having to perform costly PIKMT tallies in MCNP, the contribution of gamma heating to total heating was determined by scaling the fission heating in each region. Gamma heating fractions had been shown previously to be roughly constant over a given region, with values in the range of $0.88 \sim 0.90$ for the core regions and $0.25 \sim 0.27$ for the reflector regions [9]. Figure 9 is a diagram of the RELAP5 model, showing the inlet (140) and outlet (160) plenums, the inner (145) and outer (142) reflectors, and the three fuel regions $(152,154,156)$. The inlet and outlet temperatures were fixed at $763 \mathrm{~K}$ and $1273 \mathrm{~K}$, respectively, and rated power of $600 \mathrm{MW}$ was assumed. The RELAP5 runs typically took around one minute.

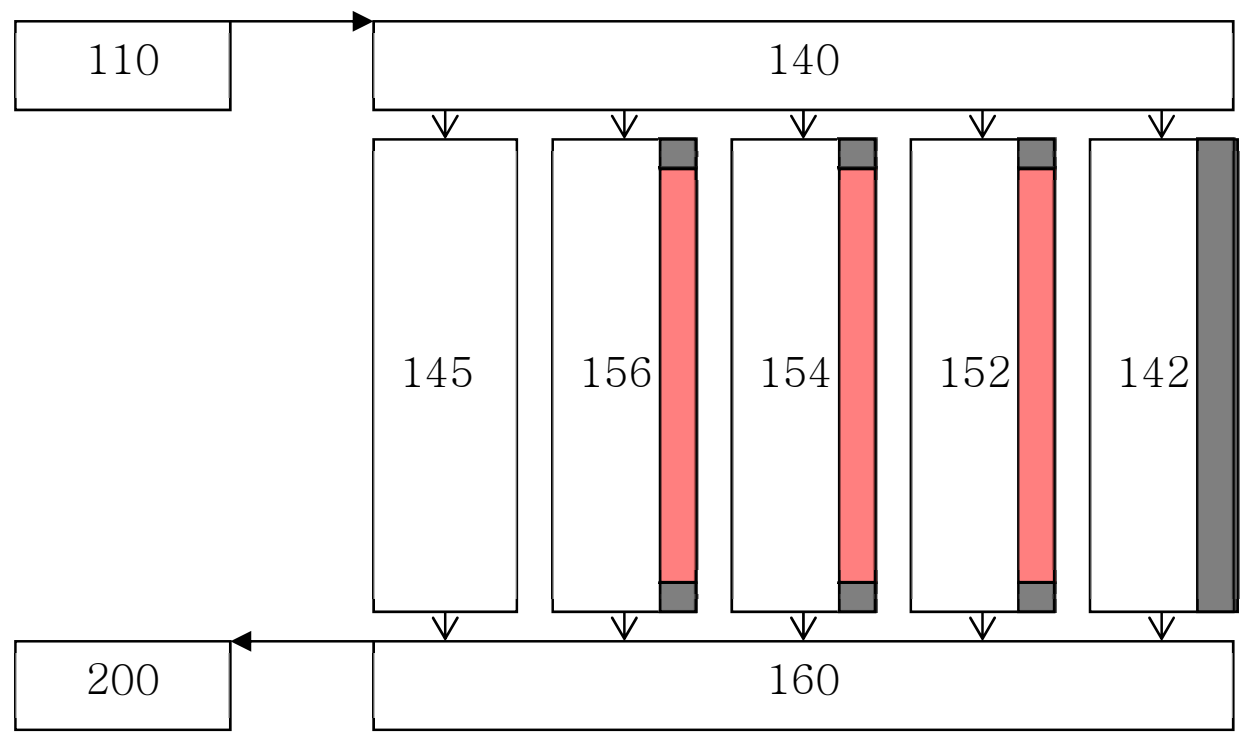

Figure 9. RELAP5 Model of the VHTR

Again using Perl scripts modified for this benchmark, updated temperatures from RELAP5 outputs were then cycled back into MCNP input files for subsequent NTH iterations to be run on the UM cluster.

Figure 10 shows the progression of keff with iteration. For both the pseudo material case and the OTF Doppler runs, convergence of keff was achieved after roughly 5 iterations. It is clear that the OTF Doppler and pseudo material simulations predict very similar results for keff as a function of the NTH iteration. Average values of keff for iterations 6 through 15 were 1.14697 for the OTF computation and 1.14694 for the pseudo material case, a difference of $3 \mathrm{pcm}$. Even 
though the pseudo material method is an approximation to the conventional ACE cross sections, the comparison is excellent.

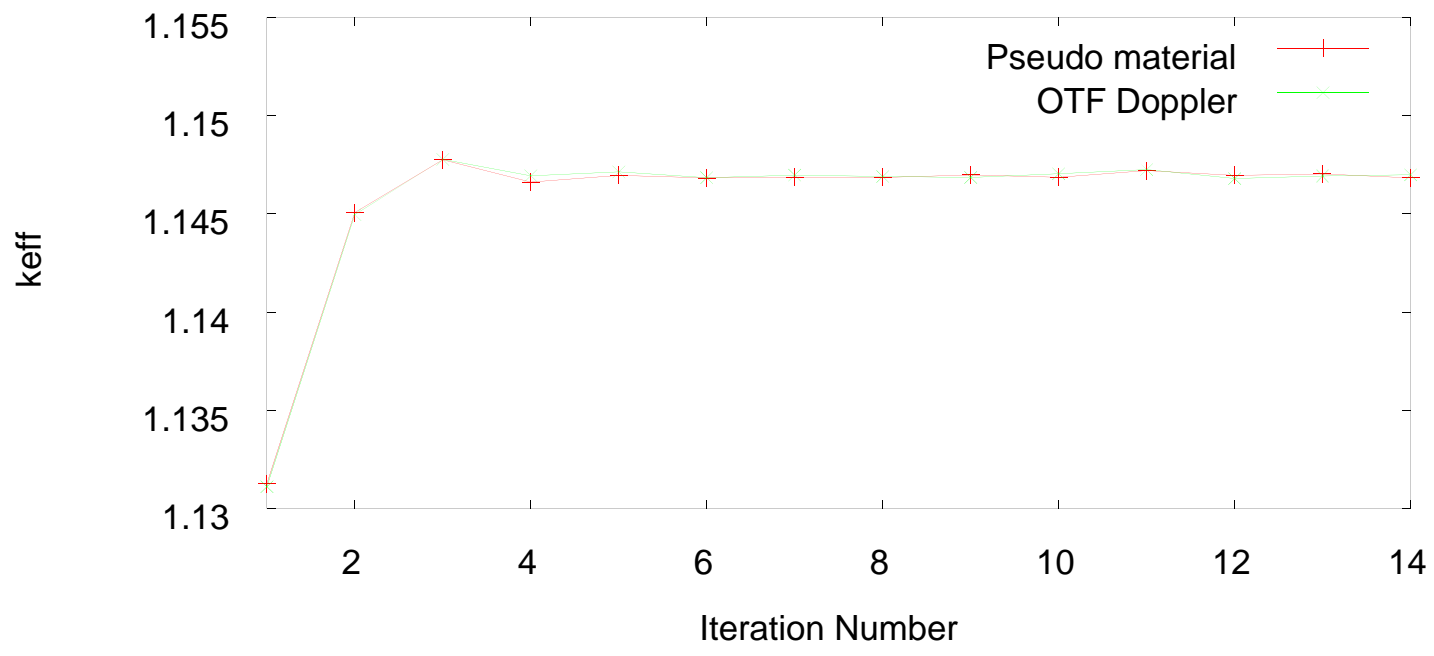

Figure 10. VHTR keff versus Cycle for OTF and Pseudo Materials

Figures 11 and 12 show the axial region temperature convergence through several NTH iterations for the middle ring core region for both the pseudo material runs and the OTF Doppler runs. Similar results were obtained for the reflector regions but are not shown in this report. Appendix B can be consulted for additional details on the results from the VHTR-2 test problem.

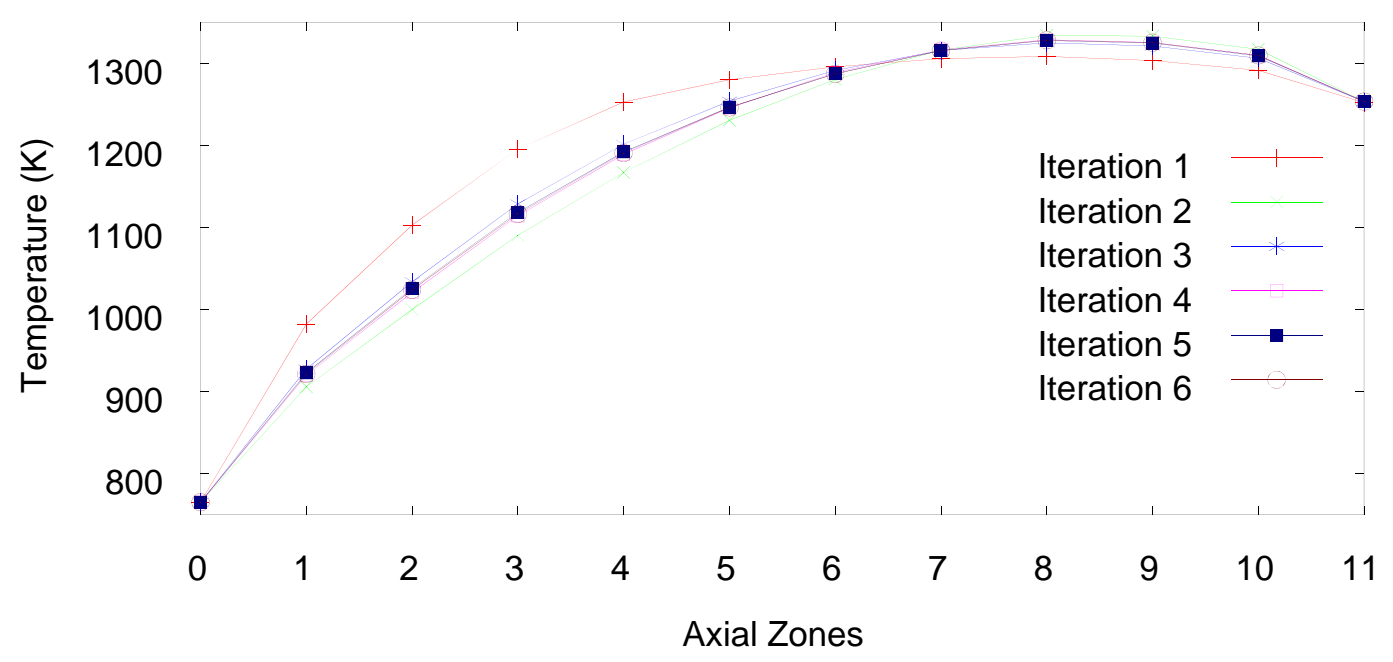

Figure 11. Axial Temperature Distribution for Middle Core Ring with Pseudo Materials

For both the reflector and the core, the OTF methodology converges the temperature distribution as fast or faster than the pseudo material method. Indeed, RMS differences (across 
the axial planes) between iterations drop to below $0.8 \mathrm{~K}$ after iteration 5 for all five regions for the OTF runs, and to below $1.06 \mathrm{~K}$ for all five regions for the pseudo material runs after iteration 6.

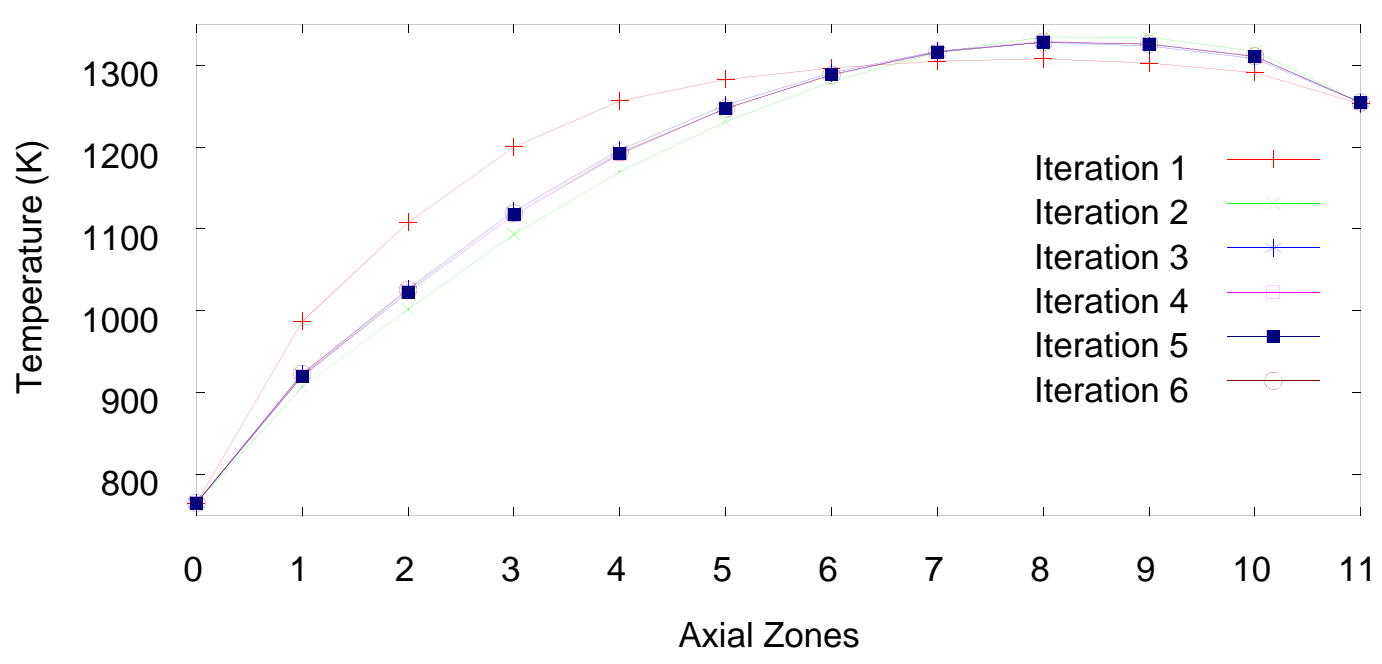

Figure 12. Axial Temperature Distribution for Middle Core Ring with OTF

Agreement between the OTF and pseudo material methods with respect to temperature was also quite good. Figure 13 shows the RELAP5 temperatures derived from the iteration 6 pseudo material simulation and the iteration 5 OTF Doppler simulations for the middle core ring. Similar results were obtained for the other regions and the details can be found in Appendix $B$. The left $y$-axis gives the actual temperature, while the right $y$-axis gives the difference between the OTF and pseudo material cases. RMS differences were less than $1.01 \mathrm{~K}$ and $0.1 \%$ for all five regions, as shown in Appendix B.

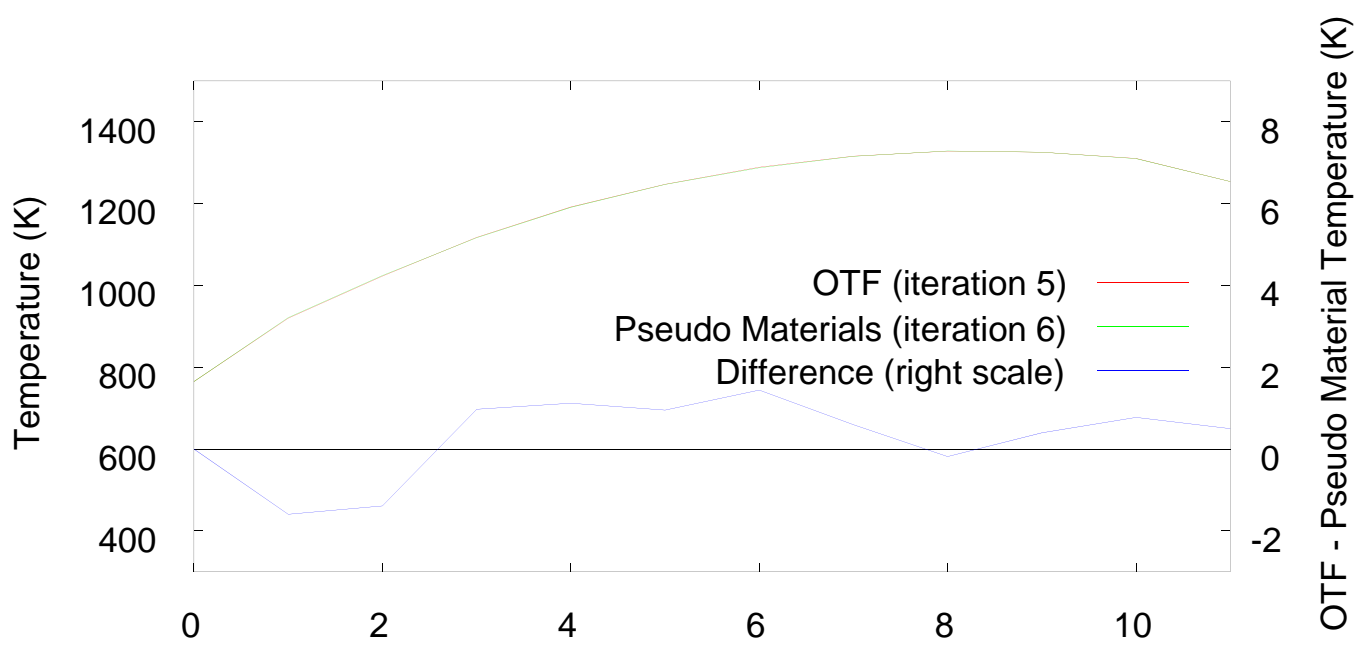

Axial Zone

Figure 13. Axial Temperature Distributions for Iterations 5-6 for Middle Core Ring 
These results clearly show that the OTF cross section capability in MCNP is generating consistent temperatures throughout the full-core geometry. The convergence trends are the same for both methods for the NTH iteration and the converged keff is essentially the same. From a performance standpoint, the computing time was about the same for OTF versus pseudo materials.

However, the input complexity is substantially reduced with OTF and the size of the cross section files is also reduced. Specifically, the size of the cross section files for the VHTR-2 test problem was $359 \mathrm{MB}$ for OTF and 1.6 GB for pseudo materials. If pseudo-materials were not used for the VHTR-2 problem, the size of the cross section files would have been 8 GB, assuming a cross section file for every $10 \mathrm{~K}$.

The substantial reductions in input complexity and cross section library size with OTF are due to the fact that the use of OTF cross sections removes the need to specify unique MCNP materials for each cell. To model temperature variation with standard MCNP runs, each cell card must specify a unique material, even when the isotopic concentration is exactly the same as that in other cells with different temperatures. This means of course that a full set of material cards, one for each isotope, must be provided for each unique temperature. With OTF, no new materials need be specified provided that the isotopic concentrations do not change. In any event, only one cross section file is needed for each isotope, regardless of the number of temperatures it may experience in the simulation.

For the VHTR-2 test problem, using OTF to model the temperatures exactly only requires the user to update the TMP values of all 60 cell cards in the MCNP input deck to reflect the new temperatures computed in RELAP. No additional input modifications are needed when using the OTF methodology. In contrast, for a conventional MCNP run, since each of the 60 cells now contains a new temperature, each material in those cells is new and so needs a new ACE material specification for each isotope in the material section of the deck. Modifications to the MCNP input deck could be minimized by re-using the ACE suffixes from the previous iteration, but in either case, a set of 300 new NJOY runs, 90 for the moderator isotopes and 210 for the fuel isotopes, would be required. This means 300 NJOY input decks would need to be modified in multiple places. Running NJOY for such a large number of fuel isotopes would add hours of computations to each feedback iteration.

\section{Summary and Conclusions}

\section{A. Summary of project}

The scientific feasibility of OTF Doppler broadening was established by Yesilyurt with his doctoral research in 2009 [3]. The current project was initiated in 2010 with the primary goal to implement OTF Doppler broadening in MCNP and test it against the conventional cross section approach by analyzing several benchmark problems including a full-core configuration with multiphysics feedback. This work has been successfully carried out. A standalone Fortran code fit_otf has been developed that generates OTF cross sections for a user-specified list of isotopes and temperature range, such as $250 \mathrm{~K}-3200 \mathrm{~K}$. The OTF library replaces MCNP cross section files (ACE files) that are broadened at user-specified problem temperatures. The OTF library is actually a set of expansion coefficients that approximates (to within a specified tolerance, typically .1\%) the temperature dependence of the cross sections at neutron energy $E$ over the specified temperature range. Thus, when a neutron of energy $E$ enters a region that is at temperature $T$, the cross sections for isotopes in that region at the exact temperature $T$ are 
immediately obtained by using a high order functional expansion for the Doppler-broadened cross sections at the energy grid points that bracket the neutron energy $\mathrm{E}$. Linear interpolation is then used to find the cross section at the energy $\mathrm{E}$.

\section{B. Concluding remarks}

This project has been completed successfully. The following observations and conclusions regarding the accuracy, efficiency, and advantages of the OTF methodology are given below:

- The OTF methodology has been successfully implemented into the MCNP Monte Carlo code. A special version of MCNP5 was developed and used for all of the OTF-based runs described in this report. The OTF Doppler broadening methodology has been implemented in a beta-release version of MCNP, MCNP6-beta-3, that will be released by RSICC in early 2013. The production release version of MCNP6 is targeted for spring/summer 2013 and will include OTF Doppler broadening.

- The OTF cross sections agree with the NJOY-based cross sections for all neutron energies and all temperatures specified by the user, e.g., 250K - 3200K. The fit_otf code generates the OTF library and can be used to generate additional OTF libraries as needed.

- Specific OTF libraries have been developed to analyze the test problems described in this report. A separate OTF library covering the temperature range $250 \mathrm{~K}-3200 \mathrm{~K}$ will be generated using fit otf that contains the following elements: $\mathrm{H}-1, \mathrm{H}-2, \mathrm{~B}-10, \mathrm{~B}-11, \mathrm{C}-12$, O-16, Si-28, Fe-54, Fe-56, Fe-57, Fe-58, Zr-90, Zr-91, Zr-92, Zr-94, Zr-96, Xe-135, Sm149, Gd-155, Gd-157, Th-232, U-233, U-234, U-235, U-238, Pu-239, Pu-240, Pu-241, and Pu-242.

- The accuracy of the OTF methodology has been assessed with several test problems by comparing MCNP runs with both conventional cross section files and OTF cross section files. The test problems include the Doppler defect reactivity benchmark suite (Mosteller suite) and two full-core VHTR configurations, including one with multiphysics coupling using RELAP5-3D/ATHENA for the thermal-hydraulic analysis. The comparison has been excellent, verifying that the OTF libraries can be used in place of the conventional ACE libraries generated at problem temperatures.

- The use of OTF cross sections greatly reduces the complexity of the input for MCNP, especially for temperature feedback calculations with many regions at different temperatures. This is due to the fact there is only one cross section file for each isotope, regardless of the number of temperatures the isotope may have in the problem. This results in an order of magnitude decrease in the number of input lines for full-core configurations, thus simplifying input preparation and reducing the potential for input errors. An estimate of the reduction factor is the number of different temperatures that are used.

- The use of OTF cross sections can lead to a substantial reduction in the size of the cross section files needed to carry out MCNP simulations with multiphysics feedback. This is due to needing only one cross section file per isotope, rather than per isotopetemperature. A ballpark estimate of the savings can be obtained by noting that a typical OTF cross section file for a given isotope may be equivalent to storing cross section files at 10-15 temperatures, depending on the isotope. For multiphysics simulations where cross sections may be needed every $10 \mathrm{~K}$, this is a huge savings in memory.

- The additional computational effort to use OTF cross sections is negligible for large problems such as the VHTR problems in this report and may be $15-20 \%$ higher for smaller problems such as the pin cell problems. 


\section{References}

[1] X-5 Monte Carlo Team, "MCNP - A General N-Particle Transport Code, Version 5, Volume I: Overview and Theory," LA-UR-03-1987, Los Alamos National Laboratory (2003).

[2] R. E. MacFarlane and D. W. Muir, "NJOY99.0 - Code System for Producing Pointwise and Multigroup Neutron and Photon Cross Sections from ENDF/B Data," PSR-480/NJOY99.00, Los Alamos National Laboratory, Los Alamos (2000).

[3] G. Yesilyurt, "Advanced Monte Carlo Methods for Analysis of Very High Temperature Reactors: On-the-Fly Doppler Broadening and Deterministic/Monte Carlo Methods," PhD thesis, Department of Nuclear Engineering and Radiological Sciences, University of Michigan, Ann Arbor, MI (2009).

[4] G. Yesilyurt, W. R. Martin, and F. B. Brown, "On-the-Fly Doppler Broadening for Monte Carlo Codes," Nucl. Sci. Eng. 171, 239-257 (2012).

[5] F. B. Brown, W. R. Martin, G. Yesilyurt, and S. Wilderman, "Progress with On-The-Fly Neutron Doppler Broadening in MCNP," Trans. Am. Nucl. Soc. 106, 508-510, Chicago, IL (June 2012).

[6] D. E. Cullen and C. R. Weisbin, "Exact Doppler Broadening of Tabulated Cross Sections", Nucl. Sci. Eng., 60, 199-229 (1976).

[7] B. T. Smith, J. M. Boyle, J. J. Dongarra, B. S. Garbow, Y. Ikebe, V. C. Klema and C. B. Moler, Matrix Eigen-system Routines - EISPACK Guide, Springer-Verlag,1976.

[8] R. D. Mosteller, "The Doppler-Defect Benchmark: Overview and Summary of Results," Proc. Joint Int. Topical Mtg on Mathematics and Computation and Supercomputing in Nuclear Applications, M\&C + SNA 2007, American Nuclear Society, Monterey, CA (2007).

[9] G. Yesilyurt, K. Banerjee, E. Villèle, J.C. Lee, and W.R. Martin, "Coupled Nuclear-ThermalHydraulic Calculations for VHTRs," Trans. Am. Nucl. Soc. 102, 519-521, San Diego, CA (June 2010).

[10] RELAP5-3D Code Development Team, "ATHENA Code Manual," INEEL-EXT-98-00834, Rev. 2.2, Idaho National Engineering and Environmental Laboratory (2003).

[11] J. L. Conlin, W. Ji, J. C. Lee, and W. R. Martin, "Pseudo Material Construct for Coupled Neutronic-Thermal-Hydraulic Analysis of VHTGR," Trans. Am. Nucl. Soc. 92, 225 (2005).

[12] Perl Programming Language, www.perl.org.

\section{Students Supported by the Contract}

The following students were supported by the grant:

- Bonita Goh - MS student.

- Andrew Pavlou - MS student.

- Eva Sunny - PhD student.

\section{Publications Directly Related to the Contract}

The following publications pertain to research supported by this grant:

[1] F. B. Brown, W. R. Martin, G. Yesilyurt, and S. Wilderman, "Progress with On-The-Fly Neutron Doppler Broadening in MCNP," Trans. Am. Nucl. Soc. 106, 508-510, Chicago, IL (June 2012).

[2] E. E. Sunny and W. R. Martin, "On-The-Fly Generation of Differential Resonance Scattering PDF," presented at the Winter Meeting of the American Nuclear Society, San Diego, CA (November 2012). 
[3] F. B. Brown, W. R. Martin, G. Yesilyurt, and S. Wilderman, "Implementation of On-The-Fly Neutron Doppler Broadening in MCNP," to be submitted to MC2013, ANS M\&C Division Topical Conference, Sun Valley, ID (May 2013).

\section{Reports and other Publications}

[1] G. Yesilyurt, "Implementation of On-the-Fly Doppler Broadening in MCNP for Multiphysics Simulation of Nuclear Reactors," ANL/NE-11-55, Argonne National Laboratory, October 28, 2011. (Attached as Appendix A.)

\section{List of Appendices}

Appendix A. Implementation of On-the-Fly Doppler Broadening in MCNP for Multiphysics Simulation of Nuclear Reactors," ANL/NE-11-55

Appendix B. Comparison of MCNP Runs with OTF versus ACE Cross Sections 
ANL/NE-11-55

\section{Implementation of On-the-Fly Doppler Broadening in MCNP5 for Multiphysics Simulation of Nuclear Reactors}

by

G. Yesilyurt

Nuclear Engineering Division, Argonne National Laboratory

October 28, 2011 


\section{Table of Contents}

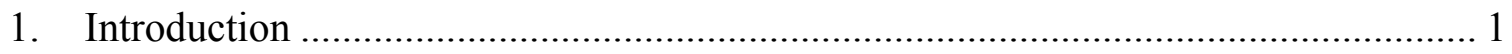

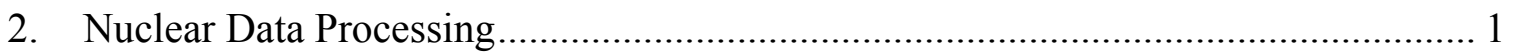

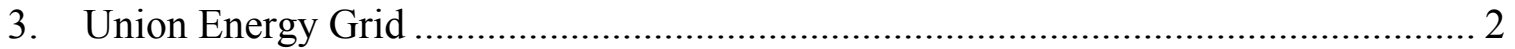

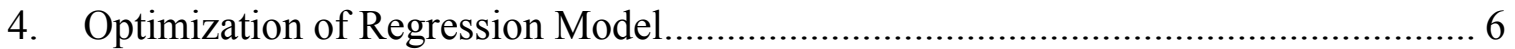

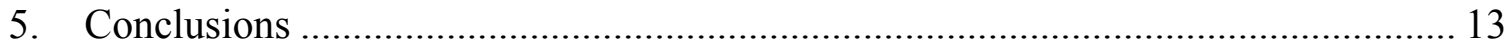

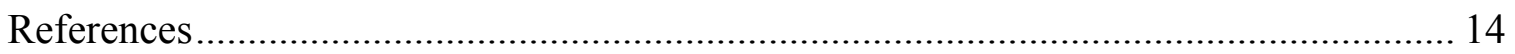

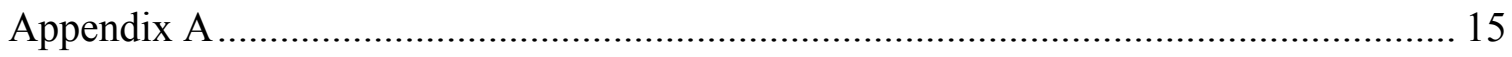




\section{List of Tables}

Table 1. Microscopic cross section descriptions.......................................................... 2

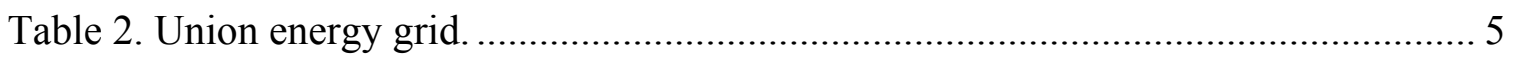

Table 3. Number of cross sections in different error intervals for different type of reactions and number of constants in the regression model. .................................. 9

Table 4. Number of cross sections in different error intervals for different type of reactions and number of constants in the regression model at $1000 \mathrm{~K}$

Table 5. Number of cross sections in different error intervals for different type of reactions and number of constants in the regression model at $2000 \mathrm{~K} \ldots \ldots \ldots \ldots \ldots \ldots . . . . . . . . . .13$ 


\section{List of Figures}

Figure 1. FT calculation for a given energy grid interval. ........................................ 2

Figure 2. Evolution of energy grid structure with temperature. ................................... 3

Figure 3. The algorithm to construct the union energy grid. ........................................ 4

Figure 4. Comparison of maximum relative error for $U-238$ capture cross section between

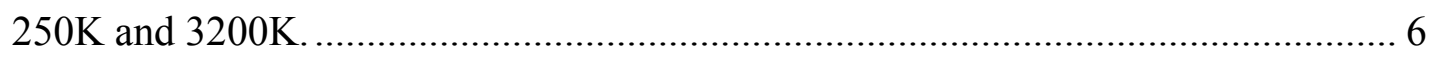

Figure 5. Comparison of maximum relative error for U-238 scattering cross section

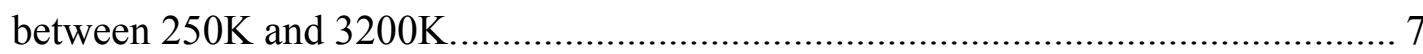

Figure 6. Comparison of maximum relative error for U-238 fission cross section between

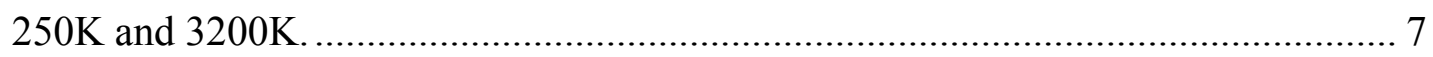

Figure 7. Comparison of maximum relative error for U-238 total cross section between

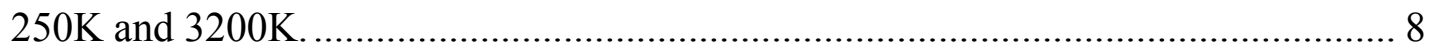

Figure 8. Residual scatter as a function of temperature at $6.67 \mathrm{eV}$ for $\mathrm{U}-238 \ldots \ldots \ldots \ldots . . .10$

Figure 9. A comparison of the total cross sections between the ADC and regression

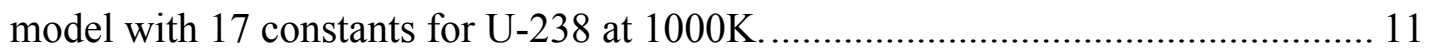

Figure 10. A comparison of scattering cross sections between the ADC and regression model with 17 constants for $\mathrm{U}-238$ at $1000 \mathrm{~K}$ 


\section{Introduction}

Effort has been made to determine a new common (union) energy grid for U-238 for a temperature range of $250 \mathrm{~K}-3200 \mathrm{~K}$. Since the energy grid structure changes with the temperature, a union energy grid is required for a given temperature range of interest. The number of coefficients in the on-the-fly (OTF) regression model ${ }^{1}$ was also optimized so that the maximum relative error for all four cross sections $\left(\sigma_{n, \gamma}, \sigma_{s}, \sigma_{f}\right.$, and $\left.\sigma_{t}\right)$ is less than $0.1 \%$ over the entire union energy grid points for the given temperature range. The optimization was performed, calculating the number of cross sections in different error intervals for different reactions and number of constants in the OTF regression model. The general form of the OTF regression model is given in Eq. (1).

$$
\sigma_{\text {tot, cap, fis }}(T) \cong \sum_{i=1}^{N} \frac{a_{i}}{T^{i / 2}}+\sum_{i=1}^{N} b_{i} T^{i / 2}+c
$$

The coefficients in Eq. (1) were calculated for all of the U-238 cross sections with different number of constants in the OTF regression model. The coefficients are going to be used in MCNP5 to perform OTF Doppler broadening during the Monte Carlo calculations.

\section{Nuclear Data Processing}

The nuclear data processing code NJOY $99.0^{2}$ was used to calculate the U-238 cross sections. The ENDF/B-VII library was utilized while processing the cross sections. The cross sections and the corresponding continuous energy structure were extracted from NJOY99.0 output files.

The total cross section in NJOY99.0 is calculated by summing up the individual reaction types as follows:

$$
\sigma_{t}=\sigma_{a}+\sigma_{s}+\sigma_{f}
$$

where $\sigma_{a}$ is defined as neutron absorption that does not include the fission:

$$
\sigma_{a}=\sigma_{n, \gamma}+\sigma_{n, p}+\sigma_{n, d}+\sigma_{n, t}+\sigma_{n, 3 H e}+\sigma_{n, \alpha}
$$

In Eq. (2), $\sigma_{f}$ is the total fission cross section, including $\sigma_{n, f}, \sigma_{n, n f}, \sigma_{n, 2 n f}$, and $\sigma_{n, 3 n f}$. Cross section descriptions and the corresponding ENDF/B-VII MT numbers are provided in Table 1. A parser script was used to extract $\sigma_{n, \gamma}, \sigma_{s}, \sigma_{f}$, and $\sigma_{t}$ from the NJOY99.0 output files. Each module in the NJOY99.0 input file produces intermediate files (Tapes) while processing the ENDF/B-VII library. The capture, scattering, and total cross sections were extracted from the ACER library (Tape-27) whereas the total fission cross 
sections were extracted from the Pendf file (Tape-23). ACER is a file format, used by the Monte Carlo code MCNP5 ${ }^{3}$. A sample NJOY99.0 input file for U-238 is provided in Appendix A.

As explained in the next section, the construction of a union energy grid is required in order to use the OTF regression model over the entire temperature range of interest for a given nuclide. The union energy grid requires a starting energy grid at $250 \mathrm{~K}$. Then, new energy grid points are added to satisfy a given fractional tolerance for the cross sections. The $0 \mathrm{~K}$ cross sections are used to Doppler broaden the cross sections and test the fractional tolerance at every temperature point between $250 \mathrm{~K}$ and $3200 \mathrm{~K}$. Therefore, the $0 \mathrm{~K}$ and $250 \mathrm{~K}$ cross sections, as well as the corresponding energy grid structures, were calculated with the help of NJOY99.0. The number of energy grid points was found to be 342,949 and 134,437 at $0 \mathrm{~K}$ and $250 \mathrm{~K}$, respectively.

Table 1. Microscopic Cross Section Descriptions.

\begin{tabular}{|r|l|}
\hline MT & Description \\
\hline 1 & Total \\
\hline 2 & Elastic scattering \\
\hline 18 & Total fission (n,fx) equal to the sum of MTs 19, 20, 21, and 38. \\
\hline 19 & $(\mathrm{n}, \mathrm{f})$ \\
\hline 20 & $(\mathrm{n}, \mathrm{n}$ 'f) \\
\hline 21 & $(\mathrm{n}, 2 \mathrm{nf})$ \\
\hline 38 & $(\mathrm{n}, 3 \mathrm{nf})$ \\
\hline 101 & Absorption: sum of MT $=102-117$ (does not include fission) \\
\hline 102 & $(\mathrm{n}, \gamma)$ \\
\hline 103 & $(\mathrm{n}, \mathrm{p})$ \\
\hline 104 & $(\mathrm{n}, \mathrm{d})$ \\
\hline 105 & $(\mathrm{n}, \mathrm{t})$ \\
\hline 106 & $(\mathrm{n}, 3 \mathrm{He})$ \\
\hline 107 & $(\mathrm{n}, \mathrm{\alpha})$ \\
\hline
\end{tabular}

\section{Union Energy Grid}

An important input parameter used by NJOY99.0 is called fractional tolerance (FT). It is defined as the relative difference in cross sections between the values of exact and linearly interpolated cross sections at mid-points between successive energy grid points.

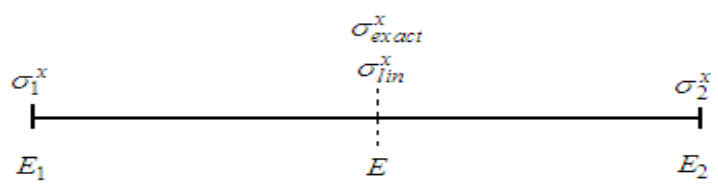

Figure 1. FT Calculation For a Given Energy Grid Interval. 
In order to construct an energy grid, the cross sections of any type $\sigma_{1}^{x}, \sigma_{2}^{x}$, and $\sigma_{\text {exact }}^{x}$ are calculated based on resonance parameters by using different multi-level representations (e.g. Multilevel Breit-Wigner, Reich-Moore, Adler-Adler, etc...) in NJOY99.0. Then the following check is performed to determine whether an additional energy grid point is required to accurately represent the cross sections between energy grid points within a given FT:

$$
\frac{\left|\sigma_{\text {exact }}^{x}-\sigma_{\text {lin }}^{x}\right|}{\sigma_{\text {exact }}^{x}}>F T
$$

If Eq. (4) is satisfied (i.e., the FT criterion is not satisfied), a new energy grid point $E$ is added halfway between $E_{1}$ and $E_{2}$. This process is performed in an iterative fashion until the convergence in Eq. (4) is reached over the entire energy grid for a given nuclide.

As shown in Figure 2, when the temperature increases, the number of energy grid points to satisfy a given FT decreases near the peak of a resonance as it smooths out, resulting in a coarser energy grid structure. On the other hand, as shown in Figure 2, when the temperature increases, more energy grid points are required for the middle and wings of a resonance to satisfy the same FT, yielding a finer energy grid structure. Although the overall effect is usually to decrease the final number of energy grid points with increasing temperature, the structure of the energy grid to satisfy a given FT depends on temperature, nuclide, and energy range within the resonance. Therefore, the construction of a union energy grid is required in order to use the OTF regression model over the entire temperature range of interest for a given nuclide.

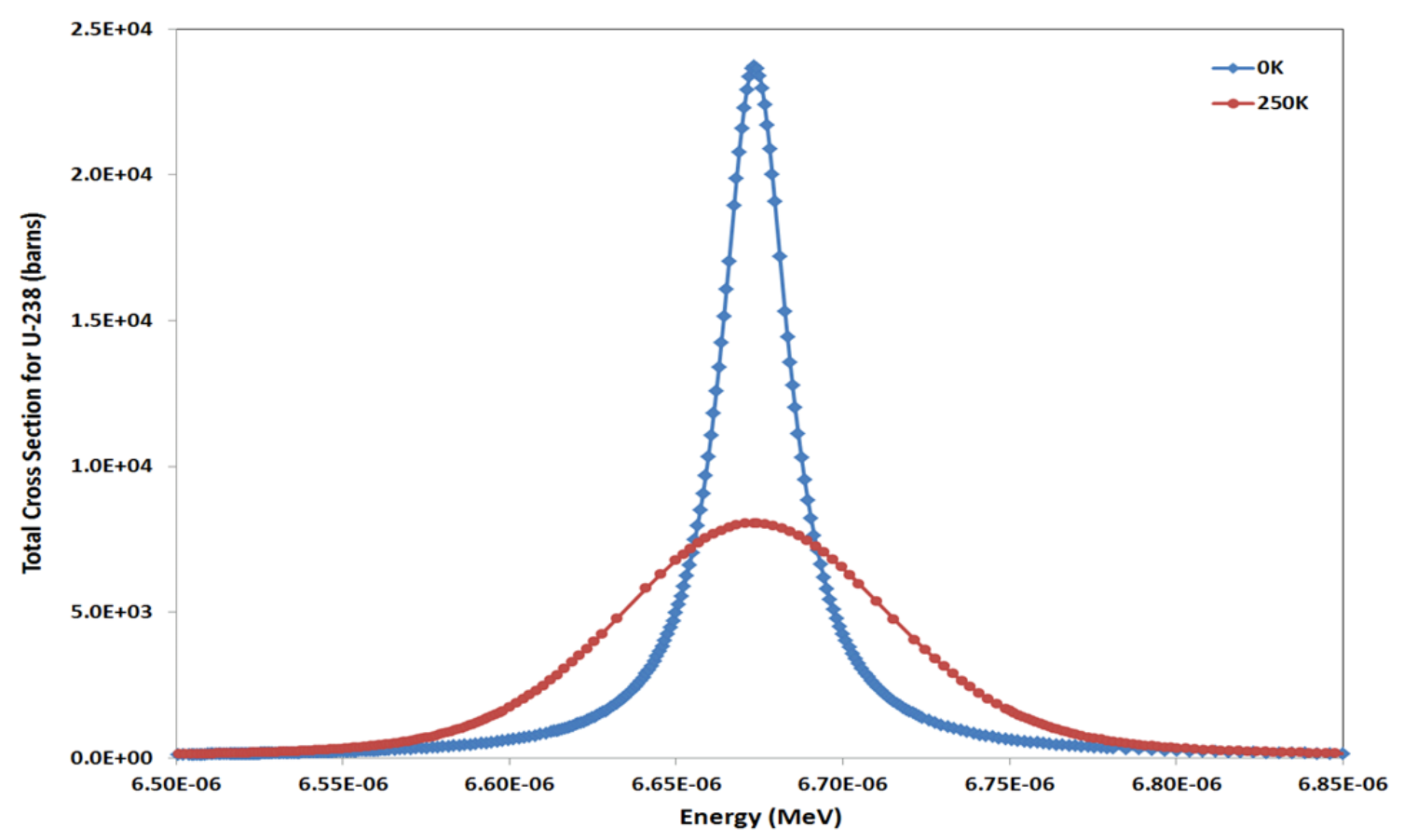

Figure 2. Evolution of Energy Grid Structure with Temperature. 
A new union energy grid was developed to allow one to calculate the constants of the OTF regression model that satisfy a given FT for the temperature range of interest [250K$3200 \mathrm{~K}]$ for U-238. This methodology includes all four cross sections $\left(\sigma_{n, \gamma}, \sigma_{s}, \sigma_{f}\right.$, and $\left.\sigma_{t}\right)$ to test the FT at every temperature point between $250 \mathrm{~K}$ and $3200 \mathrm{~K}$ to determine the final union energy grid. Cross sections were Doppler broadened at elevated temperatures, using the $0 \mathrm{~K}$ cross sections.

The algorithm depicted in Figure 3 was implemented to add new points to the $250 \mathrm{~K}$ energy grid to find a union energy grid for the entire temperature range of interest [250K$3200 \mathrm{~K}]$.

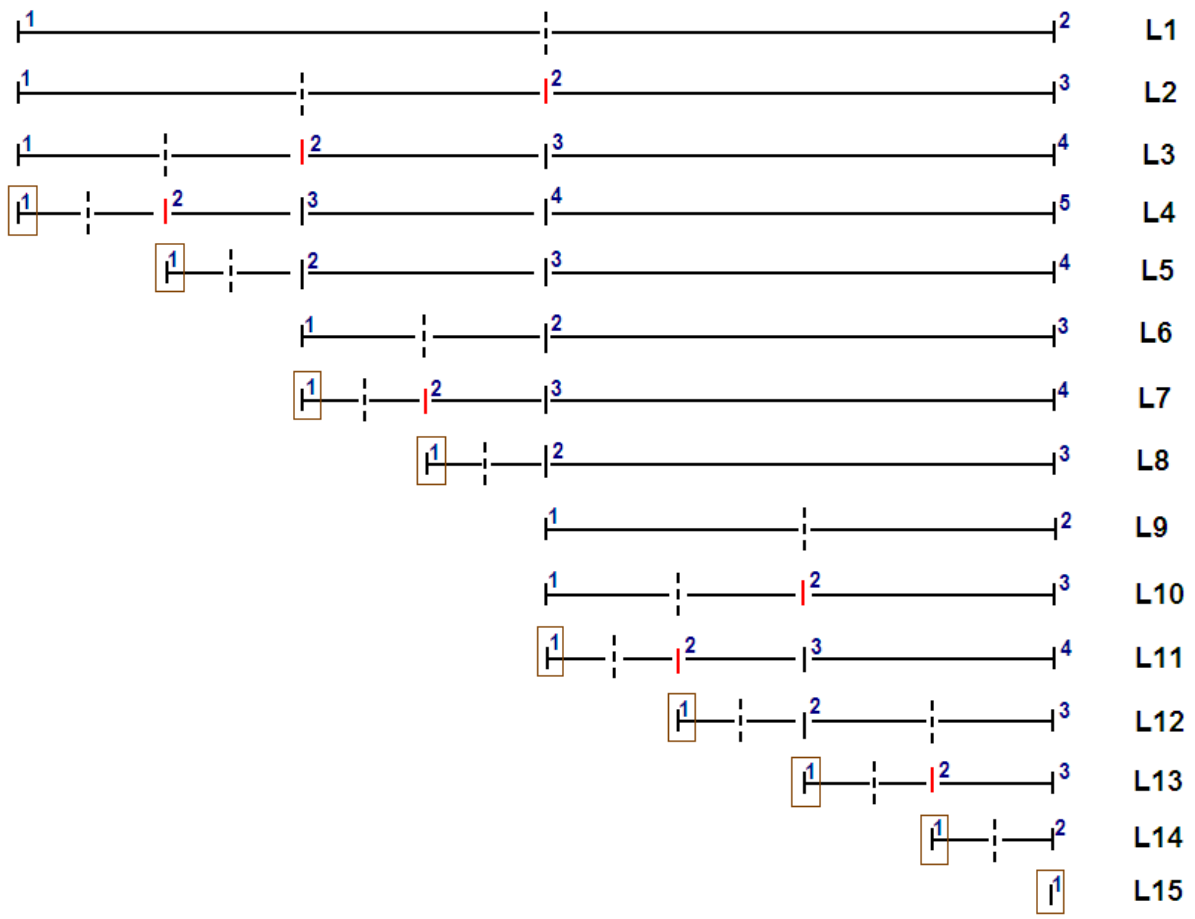

Figure 3. The Algorithm to Construct the Union Energy Grid.

In Figure 3, new energy grid points were continuously added to the starting grid at $250 \mathrm{~K}$ until convergence in FT was reached at every energy interval for the temperature range of interest. In line 1, new cross sections were calculated by applying the exact Doppler broadening equation at the middle of the successive energy grid points at every temperature point between $250 \mathrm{~K}$ and $3200 \mathrm{~K}$. The exact cross sections are then compared with the linearly interpolated cross sections at the same energy grid point for the same temperature range of interest $[250 \mathrm{~K}-3200 \mathrm{~K}]$. In this case, the cross sections, at least for one of the temperature points, were not within the given FT, so a new energy point was added to the original grid, marked with a red line, as seen in line 2 . The energy grid vector is updated automatically when a new point is added. In lines 2 and 3, the same methodology was applied but the convergence in FT, at least for one of the temperature points, was not reached so additional energy points were added. In line 4, it was found that the first energy interval converged therefore the energy grid 1, marked with a brown 
box, was recorded in a separate vector and deleted from the energy grid vector. The iteration is performed until one energy grid point is left in the energy grid vector. The energy grid points, recorded in the separate vector, represent the final union energy grid for the given nuclide and temperature range of interest.

The union energy grid cannot be generated by cross section processing codes such as NJOY. Therefore, a C ++ code, named Auxiliary Doppler Code (ADC), was implemented to determine the union energy grid and the corresponding cross sections for a temperature range of interest and a given nuclide. The ADC code was tested against NJOY at different reference temperature points for several resonance absorbers, and it was found that ADC is within 8 significant digits of NJOY. The ADC code is required to preprocess the $0 \mathrm{~K}$ cross section data for the temperature range of interest to find a union energy grid and the corresponding cross sections for a given nuclide.

It should be noted that the number of energy grid points at a given temperature can become very large for some nuclides that have narrow, high-energy resonances. This problem was solved in NJOY as follows. If the contribution to the resonance integral from any one interval is small, the interval is declared converged. This residual resonance integral error was set at 0.001 barns in NJOY for a FT of $0.1 \%$. Since important resonance integrals vary from a few barns to a few hundred barns, this yields an error in the resonance integral of less than $0.1 \%$. This methodology was also implemented in ADC.

Table 2 shows the extra grid points that were added to the starting energy grid at $250 \mathrm{~K}$. All four cross sections $\left(\sigma_{n, \gamma}, \sigma_{s}, \sigma_{f}\right.$, and $\left.\sigma_{t}\right)$ were used to test the given FT at every $50 \mathrm{~K}$ between $250 \mathrm{~K}$ and $3200 \mathrm{~K}$ to determine the final union grid. As stated in the previous section, the initial starting grid at $250 \mathrm{~K}$ has 134,437 energy points.

Table 2. Union energy grid.

\begin{tabular}{|l|r|}
\hline Reaction type & Number of union energy grid points for 250K-3200K \\
\hline Capture & 138,080 \\
\hline Scattering & 143,216 \\
\hline Fission & 143,574 \\
\hline Total & 145,303 \\
\hline
\end{tabular}

As seen in Table 2, the number of union energy grid points was found to be 145,303 for the temperature range of $250 \mathrm{~K}-3200 \mathrm{~K}$. In other words, 10,866 new energy points were added to the initial starting energy grid at $250 \mathrm{~K}$ after sweeping through all reaction types. The order of reaction types is given in Table 2 while testing the FT. 


\section{Optimization of Regression Model}

The number of constants in the OTF regression model in Eq. (1) was optimized based on the ENDF/B-VII library. The optimization was performed, calculating the maximum relative error for all four cross sections $\left(\sigma_{n, \gamma}, \sigma_{s}, \sigma_{f}\right.$, and $\left.\sigma_{t}\right)$ so that the maximum error is less than $0.1 \%$ for the temperature range of $250 \mathrm{~K}-3200 \mathrm{~K}$. The methodology involves calculating all of the cross sections $\left(\sigma_{n, \gamma}, \sigma_{s}, \sigma_{f}\right.$, and $\left.\sigma_{t}\right)$ between $250 \mathrm{~K}$ and $3200 \mathrm{~K}$ at every $1 \mathrm{~K}$ interval for every union energy grid point. Then, linear least square fitting was applied to calculate the coefficients of the OTF regression model at every union energy grid point for every reaction type of U-238.

Different number of constants was used to determine the optimal OTF regression model in Eq. (1). Figures 4, 5, 6, and 7 show the comparison of the maximum relative error in the capture, scattering, fission, and total cross sections of U-238, respectively, for the temperature range of $250 \mathrm{~K}-3200 \mathrm{~K}$.

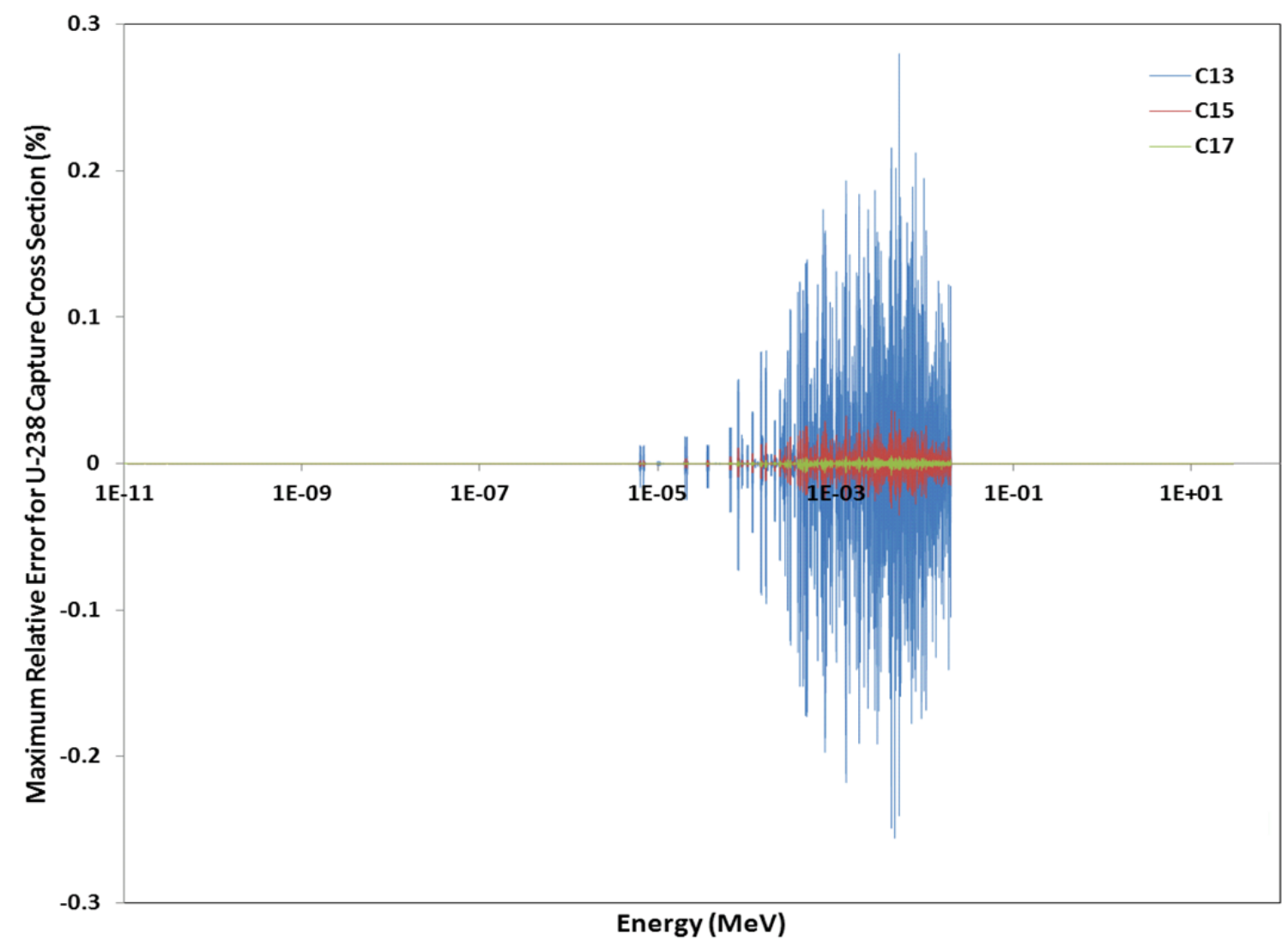

Figure 4. Comparison of Maximum Relative Error for U-238 Capture Cross Section between $250 \mathrm{~K}$ and $3200 \mathrm{~K}$. 


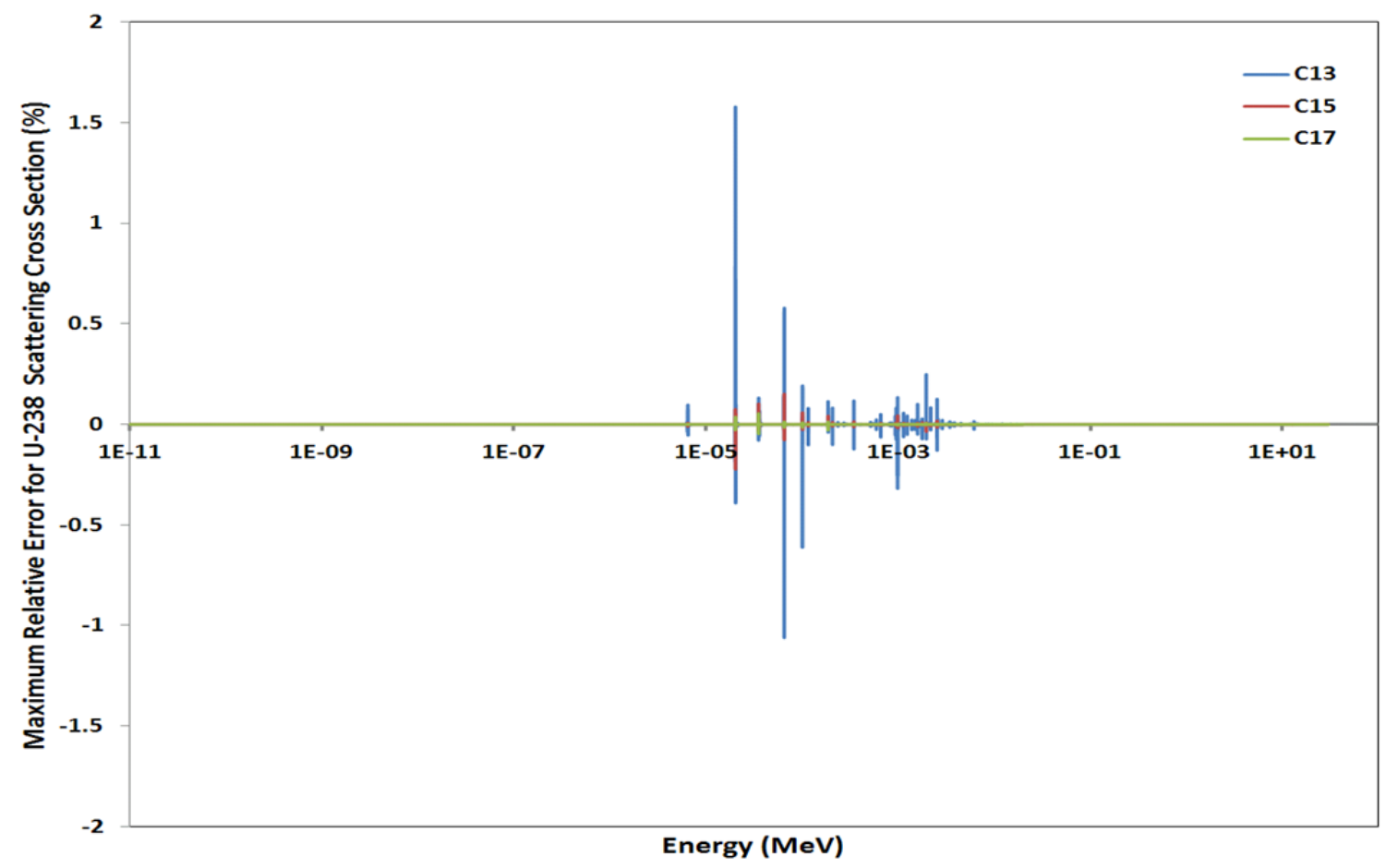

Figure 5. Comparison of Maximum Relative Error for U-238 Scattering Cross Section between $250 \mathrm{~K}$ and $3200 \mathrm{~K}$.

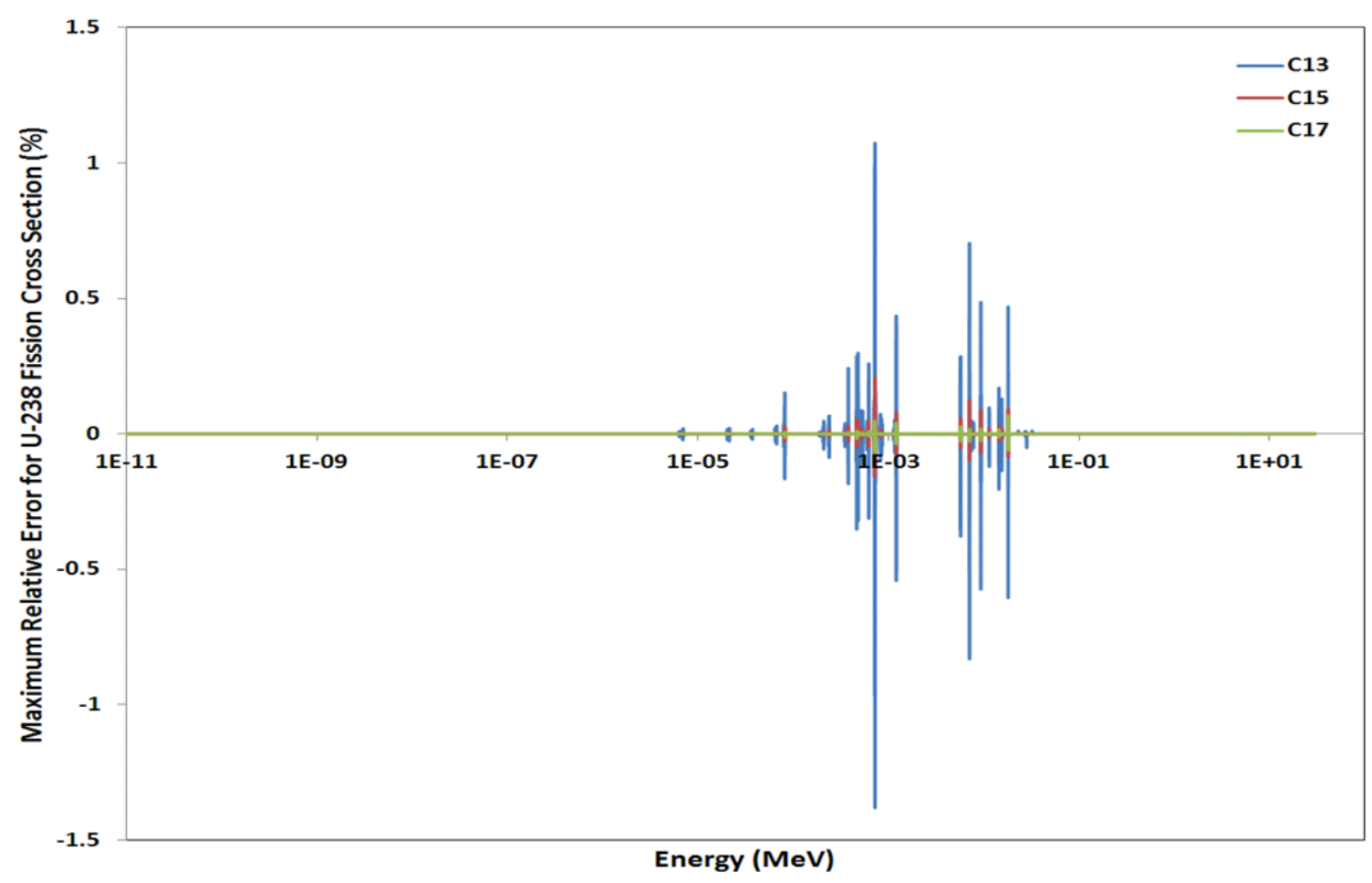

Figure 6. Comparison of Maximum Relative Error for U-238 Fission Cross Section between $250 \mathrm{~K}$ and $3200 \mathrm{~K}$. 


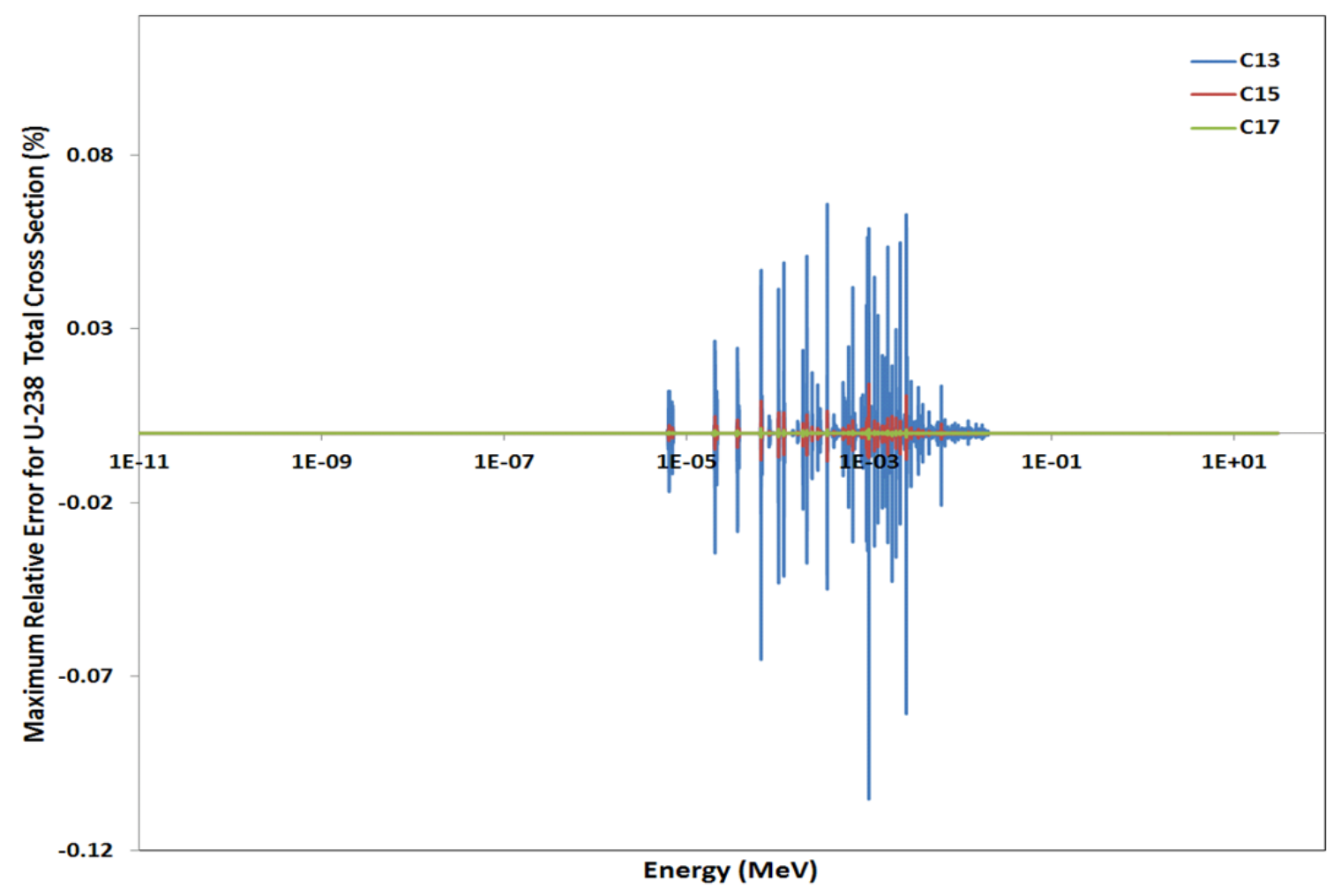

Figure 7. Comparison of Maximum Relative Error for U-238 Total Cross Section between $250 \mathrm{~K}$ and $3200 \mathrm{~K}$.

Table 3 shows the number of cross sections at the union energy grid points in different error intervals for different reactions and different number of constants in the regression model. This is a very clear picture of how cross sections are scattered into different error intervals. As seen in Table 3, there are several hundreds of cross sections between the $0.1 \%$ and $1 \%$ error interval for all reaction types when thirteen constants are used in the OTF regression model. There are only several tens of cross sections between $0.1 \%$ and $1 \%$ error interval for scattering and fission reactions when fifteen constants are used. This number goes down to zero for all cross sections when seventeen constants are used in the regression model in Eq. (1), providing an error less than $0.1 \%$. 
Table 3. Number of Cross Sections in Different Error Intervals for Different Type of Reactions and Number of Constants in the Regression Model for U-238.

\begin{tabular}{|c|c|c|c|c|c|c|}
\hline & \multicolumn{3}{|c|}{ Capture } & \multicolumn{3}{|c|}{ Scattering } \\
\hline \multirow[t]{2}{*}{ Error Interval (\%) } & \multicolumn{3}{|c|}{ Number of Cross Sections } & \multicolumn{3}{|c|}{ Number of Cross Sections } \\
\hline & C13 & C15 & C17 & C13 & C15 & C17 \\
\hline$\sigma \geq 1 \mathrm{e}-00$ & 0 & 0 & 0 & 15 & 0 & 0 \\
\hline $1 \mathrm{e}-00>\sigma \geq 1 \mathrm{e}-01$ & 600 & 0 & 0 & 192 & 25 & 0 \\
\hline $1 \mathrm{e}-01>\sigma \geq 1 \mathrm{e}-02$ & 13,853 & 1,255 & 0 & 1,230 & 273 & 69 \\
\hline $1 \mathrm{e}-02>\sigma \geq 1 \mathrm{e}-03$ & 29,514 & 16,053 & 4,279 & 6,174 & 1,266 & 379 \\
\hline $1 \mathrm{e}-03>\sigma \geq 1 \mathrm{e}-04$ & 52,127 & 31,728 & 21,331 & 33,180 & 7,153 & 1,567 \\
\hline $1 \mathrm{e}-04>\sigma \geq 1 \mathrm{e}-05$ & 36,428 & 56,166 & 44,133 & 52,540 & 38,604 & 12,777 \\
\hline $1 \mathrm{e}-05>\sigma \geq 1 \mathrm{e}-06$ & 8,213 & 31,764 & 62,394 & 34,786 & 65,406 & 80,958 \\
\hline $1 \mathrm{e}-06>\sigma$ & 4,568 & 8,337 & 13,166 & 17,186 & 32,576 & 49,553 \\
\hline \multirow[t]{2}{*}{ TOTAL } & 145,303 & 145,303 & 145,303 & 145,303 & 145,303 & 145,303 \\
\hline & \multicolumn{3}{|c|}{ Fission } & \multicolumn{3}{|c|}{ Total } \\
\hline \multirow[t]{2}{*}{ Error Interval (\%) } & \multicolumn{3}{|c|}{ Number of Cross Sections } & \multicolumn{3}{|c|}{ Number of Cross Sections } \\
\hline & C13 & C15 & C17 & C13 & C15 & C17 \\
\hline$\sigma \geq 1 \mathrm{e}-00$ & 3 & 0 & 0 & 0 & 0 & 0 \\
\hline $1 \mathrm{e}-00>\sigma \geq 1 \mathrm{e}-01$ & 663 & 16 & 0 & 3 & 0 & 0 \\
\hline $1 \mathrm{e}-01>\sigma \geq 1 \mathrm{e}-02$ & 3,644 & 1,712 & 1,033 & 1,177 & 9 & 0 \\
\hline $1 \mathrm{e}-02>\sigma \geq 1 \mathrm{e}-03$ & 3,877 & 4,157 & 2,810 & 7,018 & 1,357 & 38 \\
\hline $1 \mathrm{e}-03>\sigma \geq 1 \mathrm{e}-04$ & 1,911 & 2,783 & 3,370 & 36,209 & 8,161 & 2,111 \\
\hline $1 \mathrm{e}-04>\sigma \geq 1 \mathrm{e}-05$ & 21,921 & 1,780 & 1,858 & 61,604 & 42,213 & 14,076 \\
\hline $1 \mathrm{e}-05>\sigma \geq 1 \mathrm{e}-06$ & 57,718 & 35,451 & 11,430 & 30,034 & 70,233 & 87,265 \\
\hline $1 \mathrm{e}-06>\sigma$ & 55,566 & 99,404 & 124,802 & 9,258 & 23,330 & 41,813 \\
\hline TOTAL & 145,303 & 145,303 & 145,303 & 14,5303 & 145,303 & 145,303 \\
\hline
\end{tabular}

In general, as seen in Table 3, the capture and total cross sections are easy to fit to the regression model in Eq. (1), whereas the scattering and fission cross sections are challenging. This might be due to the scattering dips and the very narrow and sharp fission resonances. On the other hand, when seventeen constants are used in the regression model, the error in all of the cross sections over the entire union energy grid points was less than $0.1 \%$.

As a matter of fact, as the temperature increases, the error in cross sections decreases further due to smoothing effects when the regression model is used. Figure 8 shows the residual scatter as a function of temperature at $6.67 \mathrm{eV}$ for $\mathrm{U}-238$. The residual scatter is defined as follows:

$$
\text { Residual Scatter }=\sigma^{\text {Exact }}(T)-\sigma^{\text {Model }}(T)
$$




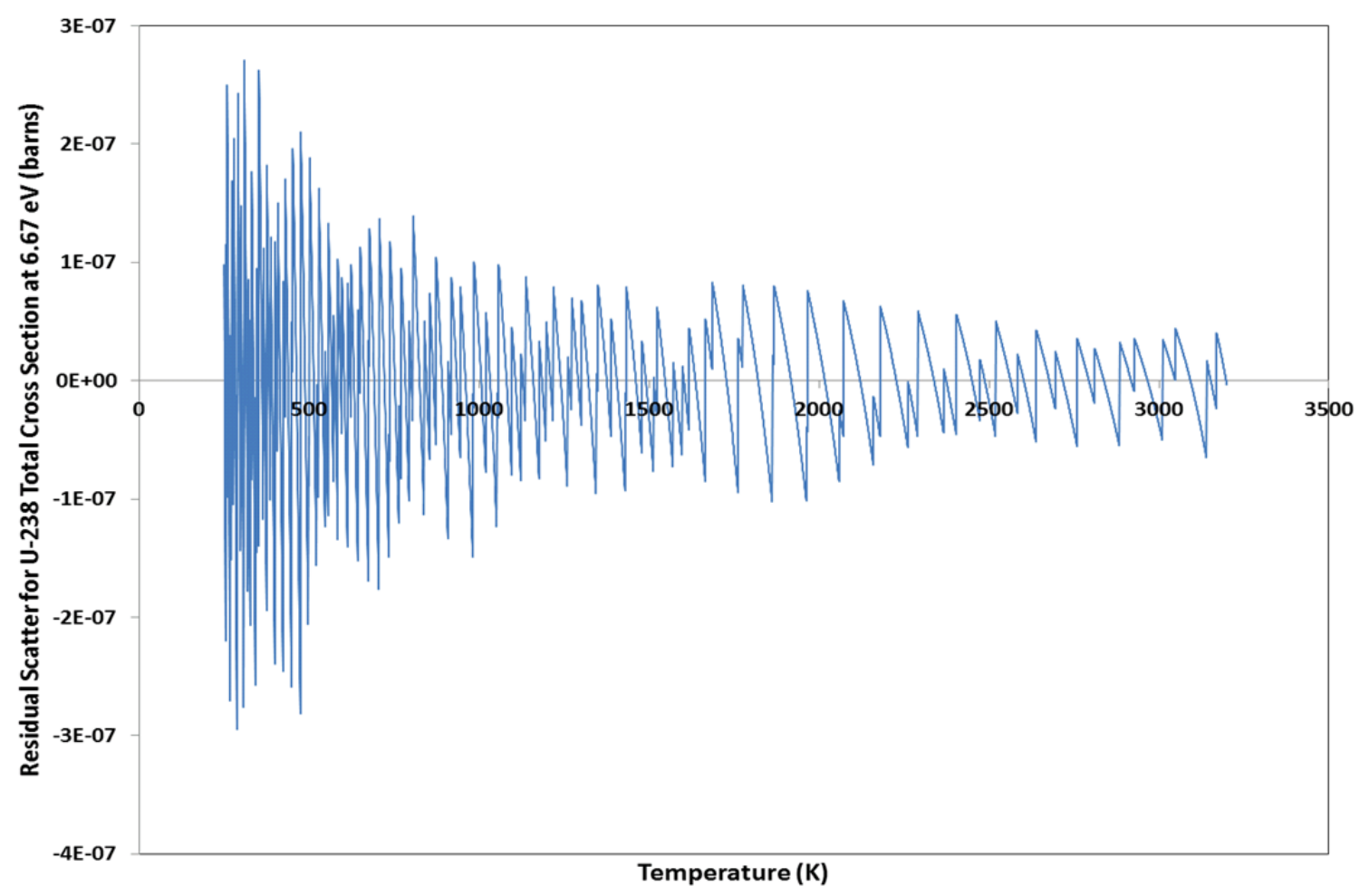

Figure 8. Residual scatter as a function of temperature at $6.67 \mathrm{eV}$ for U-238.

For realistic nuclear reactor calculations, the average fuel pin temperature is around $1000 \mathrm{~K}$ and $2000 \mathrm{~K}$ for low and high power reactors, respectively. Figure 9 shows the comparison of the total cross sections for the first important resonance of U-238 at $1000 \mathrm{~K}$. A comparison of scattering cross sections between the ADC and regression model with 17 constants is shown in Figure 10 for U-238 at 1000K. As seen in Figures 9 and 10, the regression model gets even better at elevated realistic fuel pin temperatures. 


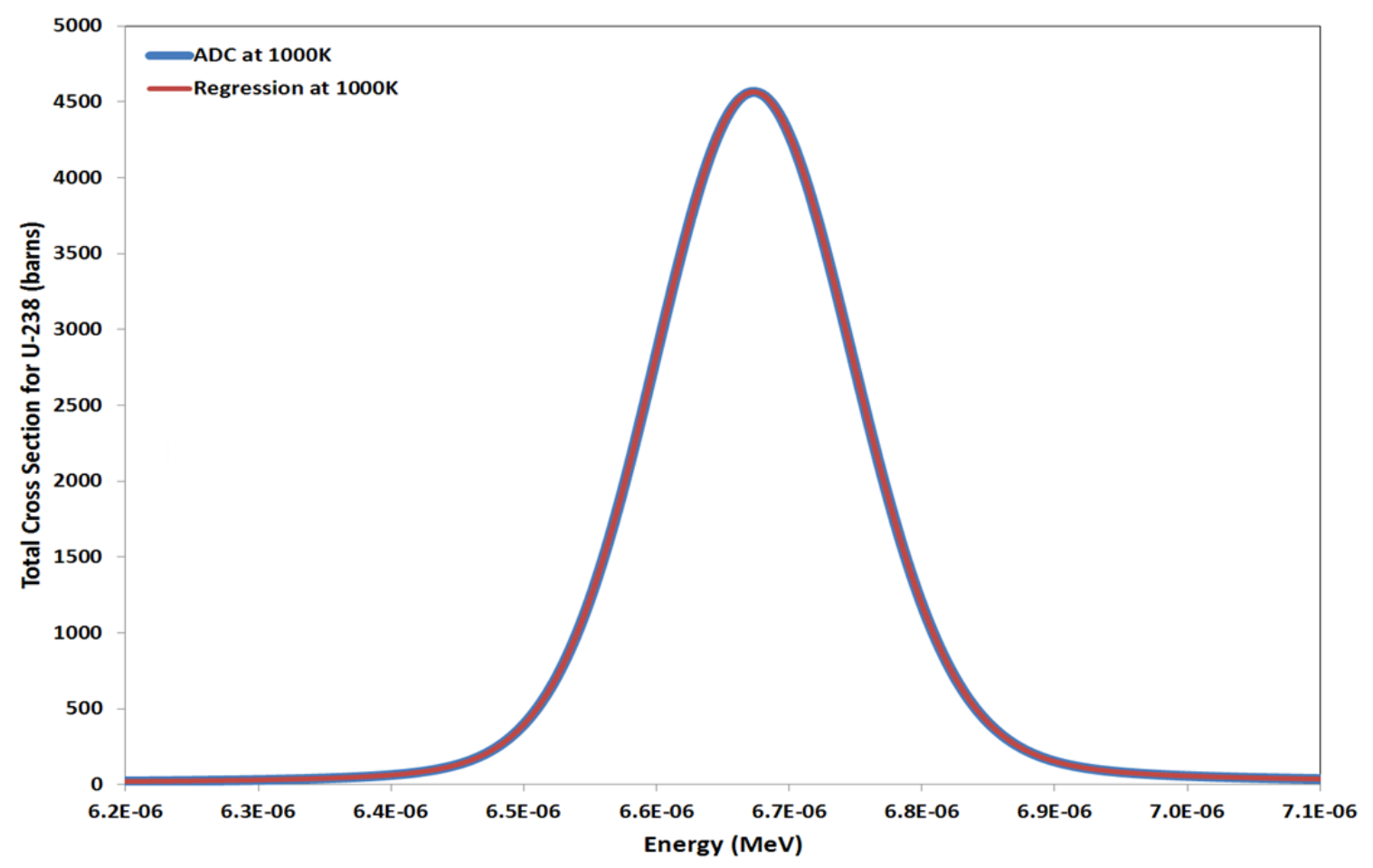

Figure 9. A Comparison of the Total Cross Sections between the ADC and Regression Model with 17 Constants for U-238 at 1000K.

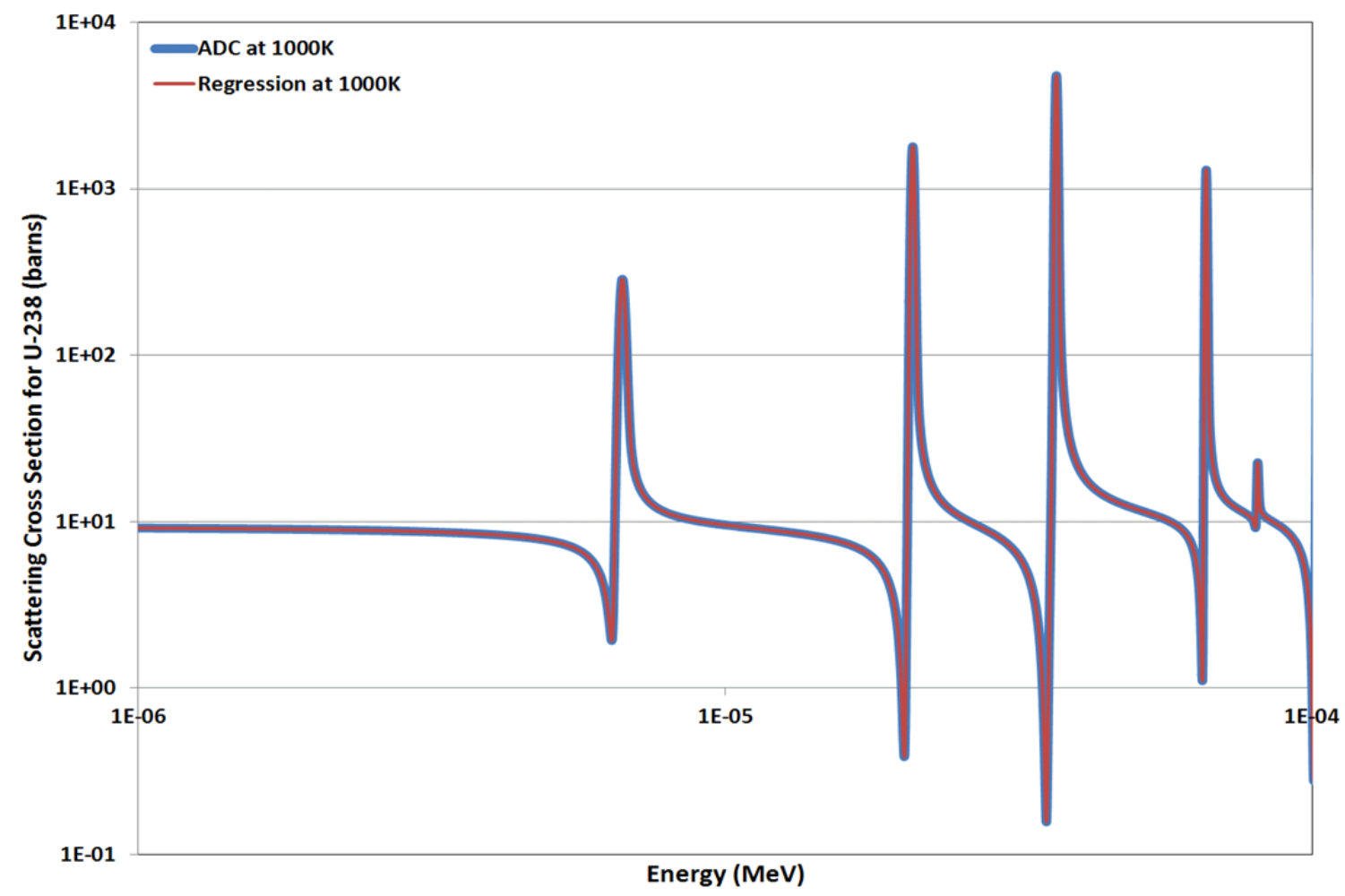

Figure 10. A Comparison of Scattering Cross Sections between the ADC and Regression Model with 17 Constants for U-238 at 1000K. 
Tables 4 and 5 show the number of cross sections at the union energy grid points in different error intervals for different reactions types and number of constants in the OTF regression model at $1000 \mathrm{~K}$ and $2000 \mathrm{~K}$, respectively.

Table 4. Number of Cross Sections in Different Error intervals for Different Type of Reactions and Number of Constants in the Regression Model at 1000K for U-238.

\begin{tabular}{|c|c|c|c|c|c|c|}
\hline & \multicolumn{3}{|c|}{ Capture } & \multicolumn{3}{|c|}{ Scattering } \\
\hline \multirow[t]{2}{*}{ Error Interval (\%) } & \multicolumn{3}{|c|}{ Number of Cross Sections } & \multicolumn{3}{|c|}{ Number of Cross Sections } \\
\hline & C13 & C15 & C17 & C13 & C15 & C17 \\
\hline$\sigma>=1 \mathrm{e}-00$ & 0 & 0 & 0 & 0 & 0 & 0 \\
\hline $1 \mathrm{e}-00>\sigma>=1 \mathrm{e}-01$ & 0 & 0 & 0 & 0 & 0 & 0 \\
\hline $1 \mathrm{e}-01>\sigma>=1 \mathrm{e}-02$ & 15 & 0 & 0 & 38 & 0 & 0 \\
\hline $1 \mathrm{e}-02>\sigma>=1 \mathrm{e}-03$ & 4,254 & 396 & 92 & 594 & 142 & 67 \\
\hline $1 \mathrm{e}-03>\sigma>=1 \mathrm{e}-04$ & 19,483 & 6,848 & 2,465 & 4,111 & 795 & 267 \\
\hline $1 e-04>\sigma>=1 e-05$ & 61,331 & 26,242 & 13,125 & 28,855 & 6,335 & 1,618 \\
\hline $1 e-05>\sigma>=1 e-06$ & 41,457 & 65,521 & 47,629 & 51,209 & 39,970 & 17,120 \\
\hline $1 \mathrm{e}-06>\sigma$ & 18,763 & 46,296 & 81,992 & 60,496 & 98,061 & 126,231 \\
\hline \multirow[t]{2}{*}{ TOTAL } & 145,303 & 145,303 & 145,303 & 145,303 & 145,303 & 145,303 \\
\hline & \multicolumn{3}{|c|}{ Fission } & \multicolumn{3}{|c|}{ Total } \\
\hline \multirow[t]{2}{*}{ Error Interval (\%) } & \multicolumn{3}{|c|}{ Number of Cross Sections } & \multicolumn{3}{|c|}{ Number of Cross Sections } \\
\hline & C13 & C15 & C17 & C13 & C15 & C17 \\
\hline$\sigma>=1 \mathrm{e}-00$ & 0 & 0 & 0 & 0 & 0 & 0 \\
\hline $1 \mathrm{e}-00>\sigma>=1 \mathrm{e}-01$ & 0 & 0 & 0 & 0 & 0 & 0 \\
\hline $1 \mathrm{e}-01>\sigma>=1 \mathrm{e}-02$ & 265 & 19 & 15 & 0 & 0 & 0 \\
\hline $1 \mathrm{e}-02>\sigma>=1 \mathrm{e}-03$ & 2,341 & 939 & 565 & 424 & 0 & 0 \\
\hline $1 \mathrm{e}-03>\sigma>=1 \mathrm{e}-04$ & 3,605 & 3,310 & 2,198 & 4,760 & 896 & 177 \\
\hline $1 e-04>\sigma>=1 e-05$ & 2,779 & 2,863 & 3,077 & 31,644 & 7,102 & 2,028 \\
\hline $1 \mathrm{e}-05>\sigma>=1 \mathrm{e}-06$ & 20,109 & 2,267 & 2,054 & 60,355 & 43,847 & 18,846 \\
\hline $1 \mathrm{e}-06>\sigma$ & 116,204 & 135,905 & 137,394 & 48,120 & 93,458 & 124,252 \\
\hline TOTAL & 145,303 & 145,303 & 145,303 & 145,303 & 145,303 & 145,303 \\
\hline
\end{tabular}


Table 5. Number of Cross Sections in Different Error Intervals for Different Type of Reactions and Number of Constants in the Regression Model at 2000K for U-238.

\begin{tabular}{|c|c|c|c|c|c|c|}
\hline & \multicolumn{3}{|c|}{ Capture } & \multicolumn{3}{|c|}{ Scattering } \\
\hline Error Interval (\%) & \multicolumn{3}{|c|}{ Number of Cross Sections } & \multicolumn{3}{|c|}{ Number of Cross Sections } \\
\hline & C13 & C15 & C17 & C13 & C15 & C17 \\
\hline$\sigma>=1 \mathrm{e}-00$ & 0 & 0 & 0 & 0 & 0 & 0 \\
\hline $1 \mathrm{e}-00>\sigma>=1 \mathrm{e}-01$ & 0 & 0 & 0 & 0 & 0 & 0 \\
\hline $1 \mathrm{e}-01>\sigma>=1 \mathrm{e}-02$ & 0 & 0 & 0 & 3 & 0 & 0 \\
\hline $1 \mathrm{e}-02>\sigma>=1 \mathrm{e}-03$ & 1,183 & 33 & 32 & 213 & 43 & 39 \\
\hline $1 \mathrm{e}-03>\sigma>=1 \mathrm{e}-04$ & 14,720 & 2,308 & 1,049 & 2,835 & 247 & 132 \\
\hline $1 \mathrm{e}-04>\sigma>=1 \mathrm{e}-05$ & 65,810 & 17,993 & 9,102 & 27,949 & 3,546 & 1063 \\
\hline $1 \mathrm{e}-05>\sigma>=1 \mathrm{e}-06$ & 45,234 & 69,903 & 47,013 & 55,619 & 34,438 & 14,993 \\
\hline $1 \mathrm{e}-06>\sigma$ & 18,356 & 55,066 & 88,107 & 58,684 & 107,029 & 129,076 \\
\hline \multirow[t]{2}{*}{ TOTAL } & 145,303 & 145,303 & 145,303 & 145,303 & 145,303 & 145,303 \\
\hline & \multicolumn{3}{|c|}{ Fission } & \multicolumn{3}{|c|}{ Total } \\
\hline \multirow[t]{2}{*}{ Error Interval (\%) } & \multicolumn{3}{|c|}{ Number of Cross Sections } & \multicolumn{3}{|c|}{ Number of Cross Sections } \\
\hline & C13 & C15 & C17 & C13 & C15 & $C 17$ \\
\hline$\sigma>=1 \mathrm{e}-00$ & 0 & 0 & 0 & 0 & 0 & 0 \\
\hline $1 \mathrm{e}-00>\sigma>=1 \mathrm{e}-01$ & 0 & 0 & 0 & 0 & 0 & 0 \\
\hline $1 \mathrm{e}-01>\sigma>=1 \mathrm{e}-02$ & 78 & 51 & 50 & 0 & 0 & 0 \\
\hline $1 \mathrm{e}-02>\sigma>=1 \mathrm{e}-03$ & 1,865 & 689 & 657 & 89 & 0 & 0 \\
\hline $1 \mathrm{e}-03>\sigma>=1 \mathrm{e}-04$ & 3,861 & 2,503 & 1,846 & 3,299 & 193 & 91 \\
\hline $1 \mathrm{e}-04>\sigma>=1 \mathrm{e}-05$ & 2,963 & 3,059 & 2,497 & 30,383 & 4,189 & 1,314 \\
\hline $1 \mathrm{e}-05>\sigma>=1 \mathrm{e}-06$ & 18,686 & 3,075 & 2,865 & 64,086 & 37,211 & 16,382 \\
\hline $1 \mathrm{e}-06>\sigma$ & 117,850 & 135,926 & 137,388 & 47,446 & 103,710 & 127,516 \\
\hline TOTAL & 145,303 & 145,303 & 145,303 & 145,303 & 145,303 & 145,303 \\
\hline
\end{tabular}

\section{Conclusions}

A new union energy grid was constructed for U-238 for the temperature range of $250 \mathrm{~K}$ $3200 \mathrm{~K}$. The number of coefficients in the OTF regression model was optimized so that the maximum relative error for all four cross sections $\left(\sigma_{n, \gamma}, \sigma_{s}, \sigma_{f}\right.$, and $\left.\sigma_{t}\right)$ is less than $0.1 \%$ over the entire union energy grid points for the temperature range of interest. The coefficient files were prepared for U-238 for the use of MCNP5 to Doppler broaden the cross sections on-the-fly during the Monte Carlo calculations. 


\section{References}

1. Yesilyurt, Gokhan, “Advanced Monte Carlo Methods for Analysis of Very High Temperature Reactors: On-the-Fly Doppler Broadening and Deterministic /Monte Carlo Methods," PhD Thesis, Department of Nuclear Engineering and Radiological Sciences, University of Michigan, Ann Arbor, Michigan (2009).

2. MacFarlane, R.E. and Muir, D.W., "NJOY99.0 - Code System for Producing Pointwi se and Multigroup Neutron and Photon Cross Sections from ENDF/B Data," PSR-480 /NJOY99.00, Los Alamos National Laboratory, Los Alamos (2000).

3. Monte Carlo Team, "A General Monte Carlo N-Particle Transport Code," Version 5, LA-UR-03-1987, Los Alamos National Laboratory (2003). 


\section{Appendix A}

The following input file was used to process the U-238 cross sections with NJOY99.0.

Each module in NJOY99.0 input file produces intermediate files (Tapes) while

processing the ENDF/B-VII library. The capture, scattering, and total cross sections were extracted from the ACER library (Tape-27) whereas the fission cross sections were extracted from the Pendf file (Tape-23). The ACER is a file format, used by the Monte Carlo code MCNP5.

echo 'U-238 (Uranium 238) Temperature dependent x-section generation'

ln -fs /Users/gyesilyurt/projects/neup/rawdata/92238.9237.v7 tape21

echo 'Running NJOY...'

cat $>$ input $<<$ EOF

reconr

2122

'pendf tape for endfb-v.0 u238 MAT: 9237 '/

$923730 /$

$0.0010 /$

'u238 MAT 9237'/

'processed by the njoy system'/

'gokhan yesilyurt, 01/13/09'/

$0 /$

broadr

212223

$9237100 /$

$0.001 /$

250.0

$0 /$

unresr

212324

9237171

250.0

1.E+10 1.E+06 1.E+05 1.E+04 1.E+03 1.E+02 5.E+01

$0 /$

purr

212425

$9237172032 /$

250.0

1.E+10 1.E+06 1.E+05 1.E+04 1.E+03 1.E+02 5.E+01

$0 /$

thermr

02526

092371611012211

250.0 


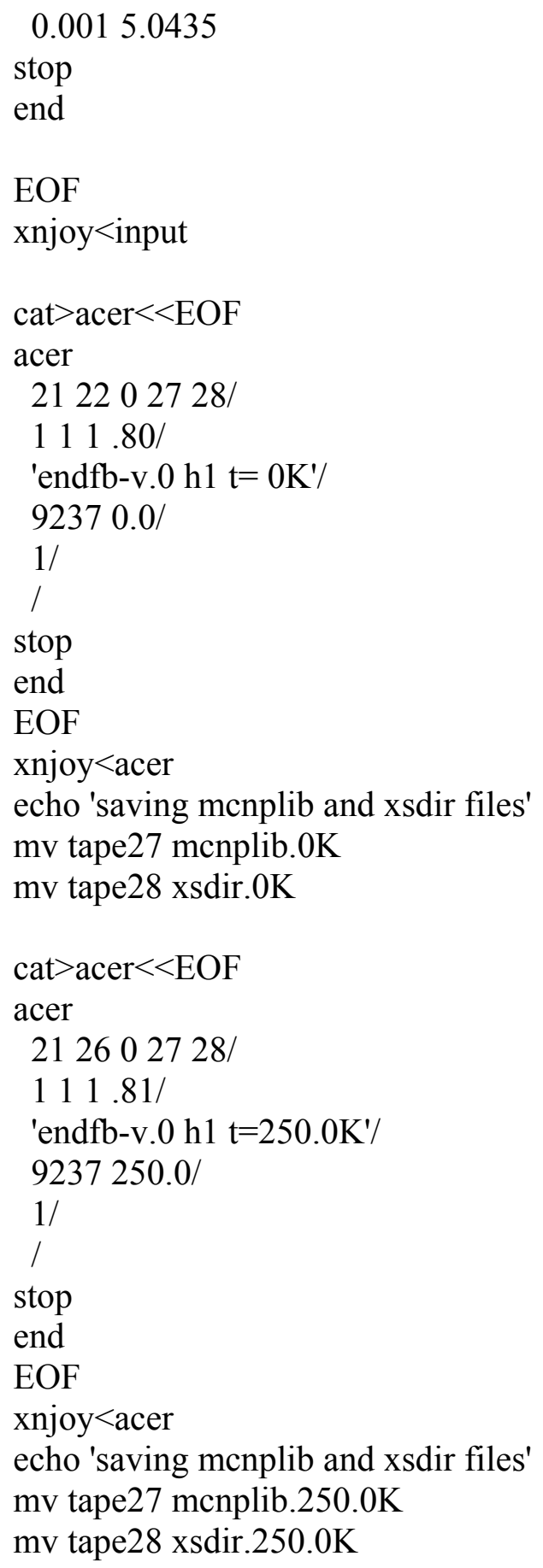




\section{Comparison of MCNP Runs with OTF versus ACE Cross Sections \\ Scott Wilderman \\ November 16, 2012}

Two problems were chosen as final benchmarks and as demonstrations of the ease of use of the OTF methodology. The first problem a coupled nuclear-thermal hydraulics calculation for a VHTR, based on that reported by Yesilyurt et al[4]. A full VHTR core consisting of an annular array of 1024 fuel blocks surrounded by moderator, shown below, was represented as 3 rings of fuel blocks situated between inner reflector and outer reflector regions.

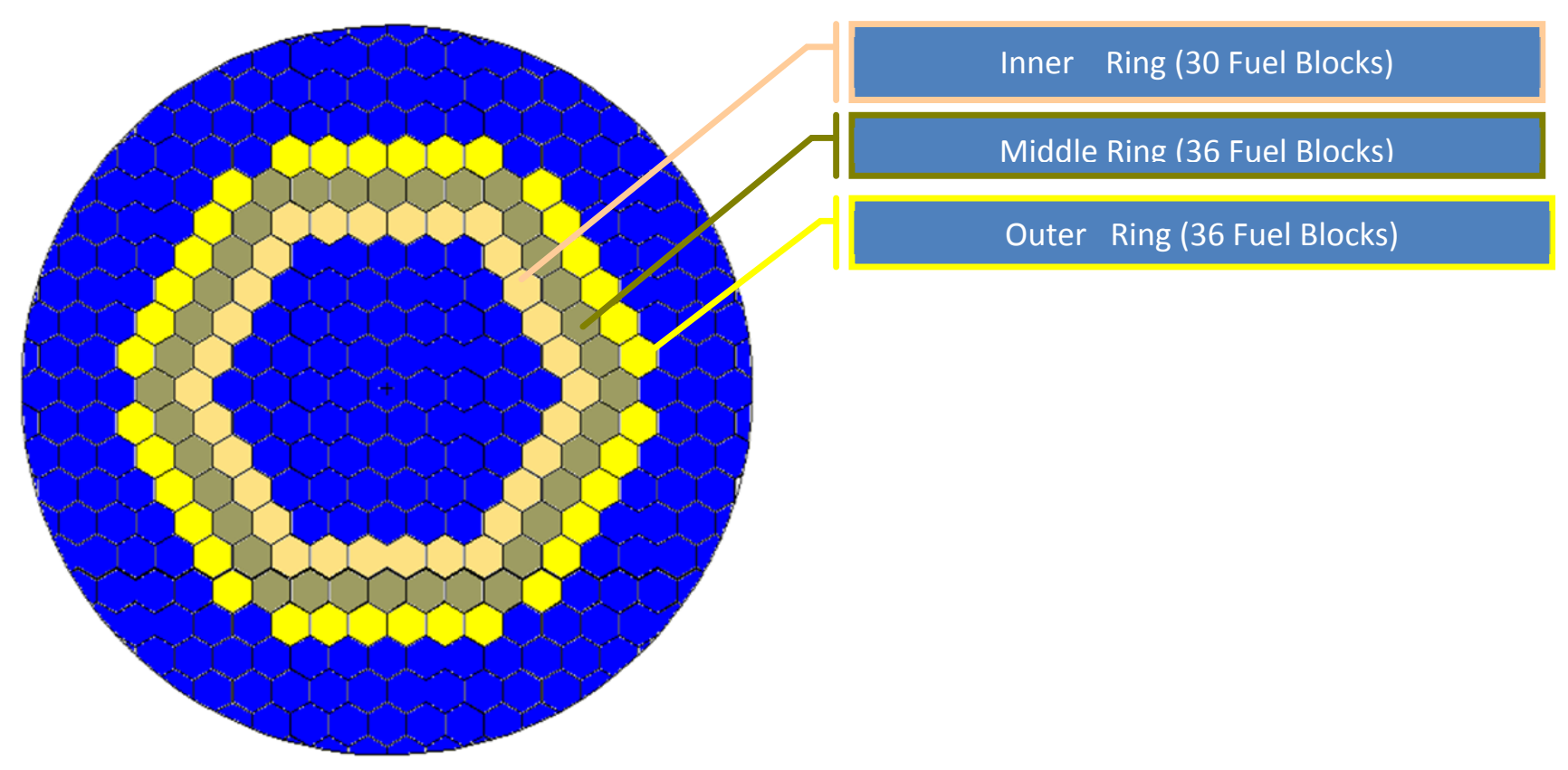

The five regions (two moderator and three fuel) were divided into 10 axial planes, with additional reflector planes at the top and bottom. Regions were assumed to consist of homogenous media with varying temperatures (60 total materials), and axial power profiles were computed using both the OTF methodology and using fixed temperatures but with cross sections interpolated using the pseudo material construct [11]. The fuel consists of TRISO particles containing $10.36 \%$ enriched $\mathrm{UO}_{2}$, silicon and graphite, and the reflectors graphite with 6.9 ppm of natural Boron.

For the pseudo material problem, endf7 mcnp5 library files were prepared using NJOY99 (update 364) for isotopes B-10, B-11, and carbon at every $100 \mathrm{~K}$ from $700 \mathrm{~K}$ to $2400 \mathrm{~K}$. For isotopes U-235, U-238, O-16, and Si-28, temperature dependent library files were generated at every $100 \mathrm{~K}$ from 900 to 2400 $\mathrm{K}$, giving a total of $125 \mathrm{mcnp}$ library files and 1.6 GB of data. For the lighter elements, generation of the data files took just several minutes, but, because of the need to generate probability tables for determining cross sections in the unresolved resonance region for the heavier materials, several hours of computation time on quad-core processors were required to prepare data for each heavy isotope.

Data files were also prepared for OTF cases in the temperature range from $600 \mathrm{~K}$ to $3000 \mathrm{~K}$, using the standard MCNP libraries at $600 \mathrm{~K}$ as the base ACER library. Again, data preparation computation times were minimal for low Z materials, increasing to approximately 40 minutes for U-235 and two hours for 
U-238, because of the large number of energy grid points. Total OTF data library size was 359 MB.

Temperatures of moderators for $S(\alpha, \beta)$ treatment were fixed at $1200 \mathrm{~K}$ in the fuel and $1000 \mathrm{~K}$ in the reflectors (note that the prior work of Yesilyur used room temperature data in all regions). Because of the temperature variation in each region, each cell in the pseudo material implementation was a unique MCNP5 input material, requiring a total of 668 cards. In contrast, the MCNP input using the OTF method required just 16 cards to specify the materials.

MCNP5 runs used 200,000 histories per cycle and 600 cycles, discarding the first 200, and required roughly 5 hours on 16 cores (using MPI) at the UM CAC high performance cluster. The performance penalty previously seen in running OTF Doppler cases with individual isotopes was not present here, probably due to a combination of overhead associated with MPI and the presence of twice as many isotopes in each cell, required when pseudo materials are used.

MCNP5 output files were transferred from the CAC cluster to a Windows PC running Cygwin Linux. Axial power fractions were extracted from MCNP5 output using perl scripts modified from earlier work. Fractional regional power data was merged with input templates to produce input files which were then passed to RELAP5-3D/ATHENA [10] to compute fuel-block average temperatures. In order to preclude having to perform costly PIKMT tallies in MCNP5, the contribution of gamma heating to total heating was determined by scaling the fission heating in each region. Gamma heating fractions had been shown previously to be roughly constant over a given region, with values in the range of $0.88 \sim 0.90$ for the core regions and $0.25 \sim 0.27$ for the reflector regions [4]. In the RELAP 5-3D computation, the core was modeled as concentric cylinders corresponding to the inner reflector, inner core ring, middle core ring, outer core ring and outer reflector. Inlet and outlet temperatures were fixed at $763 \mathrm{~K}$ and $1273 \mathrm{~K}$, respectively, and rated power of $600 \mathrm{MW}$ was assumed. RELAP5-3D runs typically took around one minute.

Again using perl scripts modified for this benchmark, updated temperatures from RELAP5-3D outputs were then cycled back into MCNP5 input files for subsequent iterations to be run on the UM CAC cluster. At each iteration, the temperature (TMP attribute) of each cell card in the input deck was updated for both the OTF run and the pseudo material treatment. While no further modifications were required for the OTF analysis, for the pseudo material cases in was necessary for each cell to determine the new ACER libraries to be accesses and the temperature-based fractional component of each constituent isotope.

\section{Results:}

The figure below shows the progression of k-effective with iteration. For both the pseudo material case and the OTF Doppler runs, convergence of k-effective was achieved after roughly 5 iterations. It is also apparent that the OTF Doppler and pseudo material simulations predict very similar results for keffective. Average values of k-effective for iterations 6 through 15 were 1.14697 for the OTF computation, and 1.14694 for the pseudo material case. 


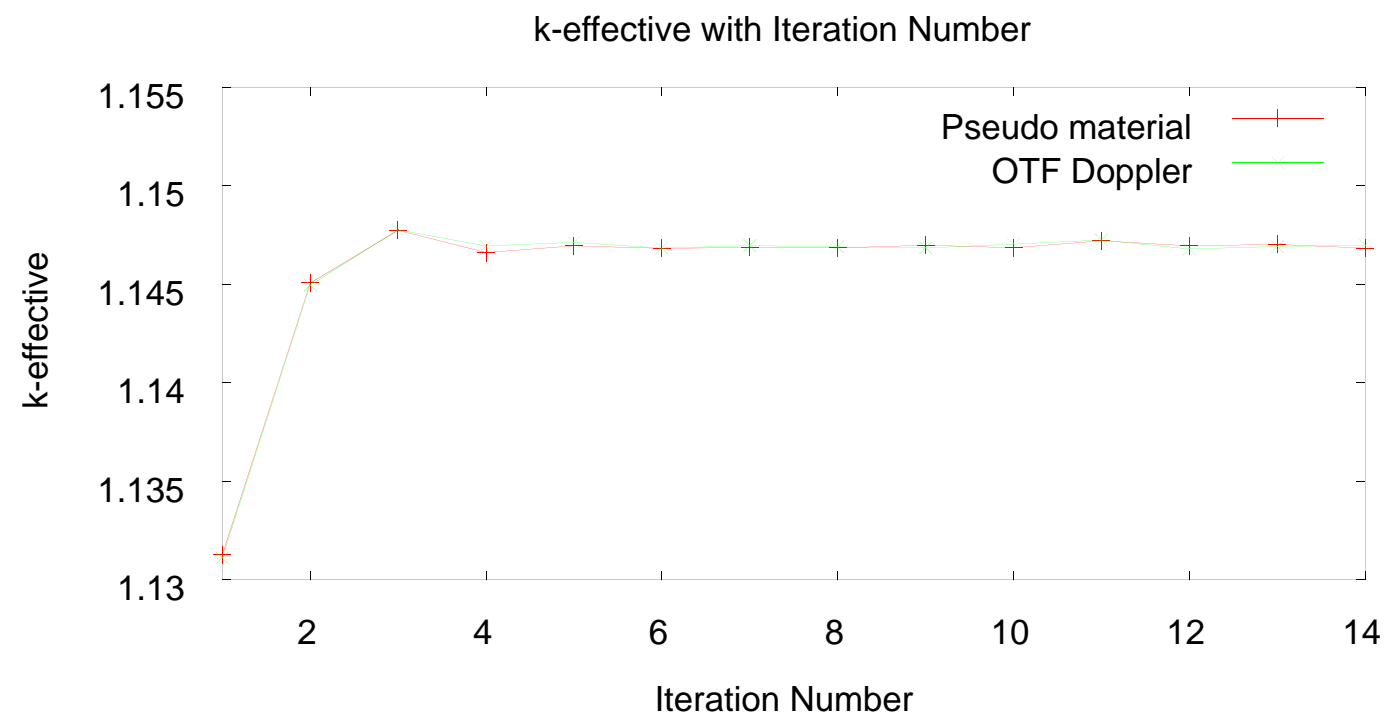

The figures below show the axial region temperature convergence through several iterations for the middle ring core region and the inner reflector for both the pseudo material runs and the OTF Doppler runs.

Temperatures in Axial Planes of the Inner Reflector Zone, Pseudo Material Runs

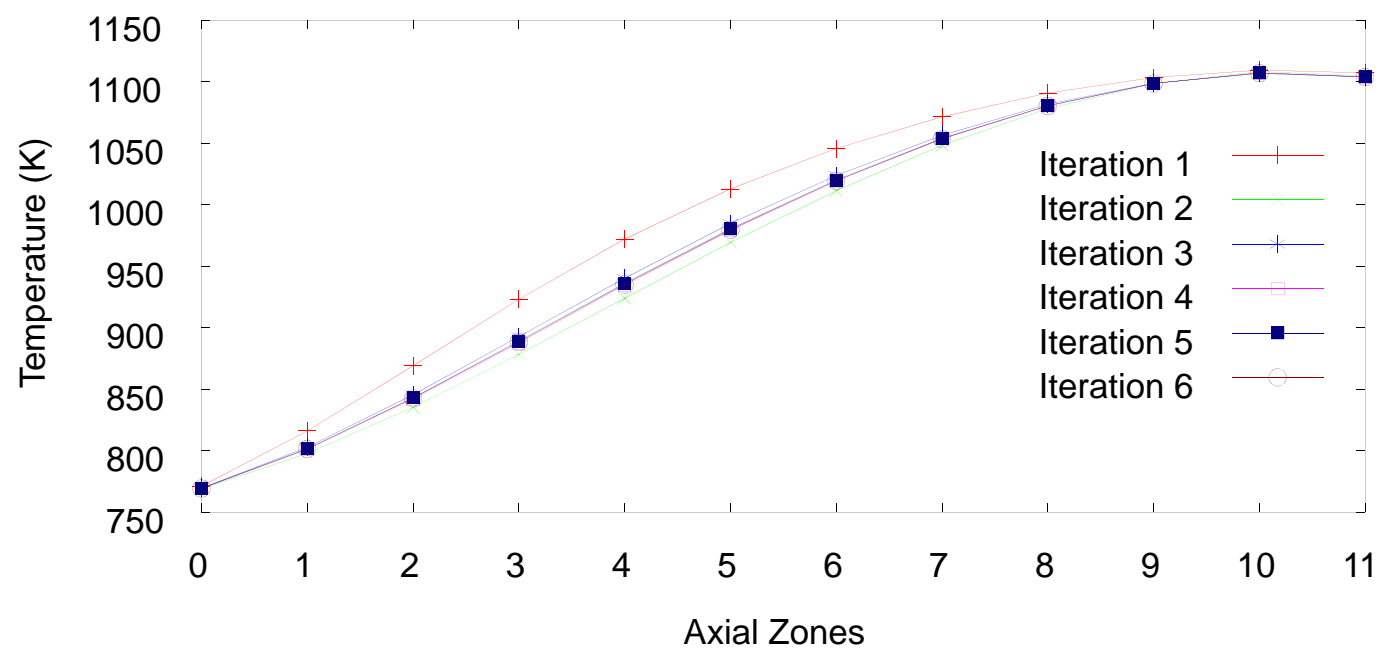


Temperatures in Axial Planes of the Inner Reflector Zone, OTF Doppler Runs

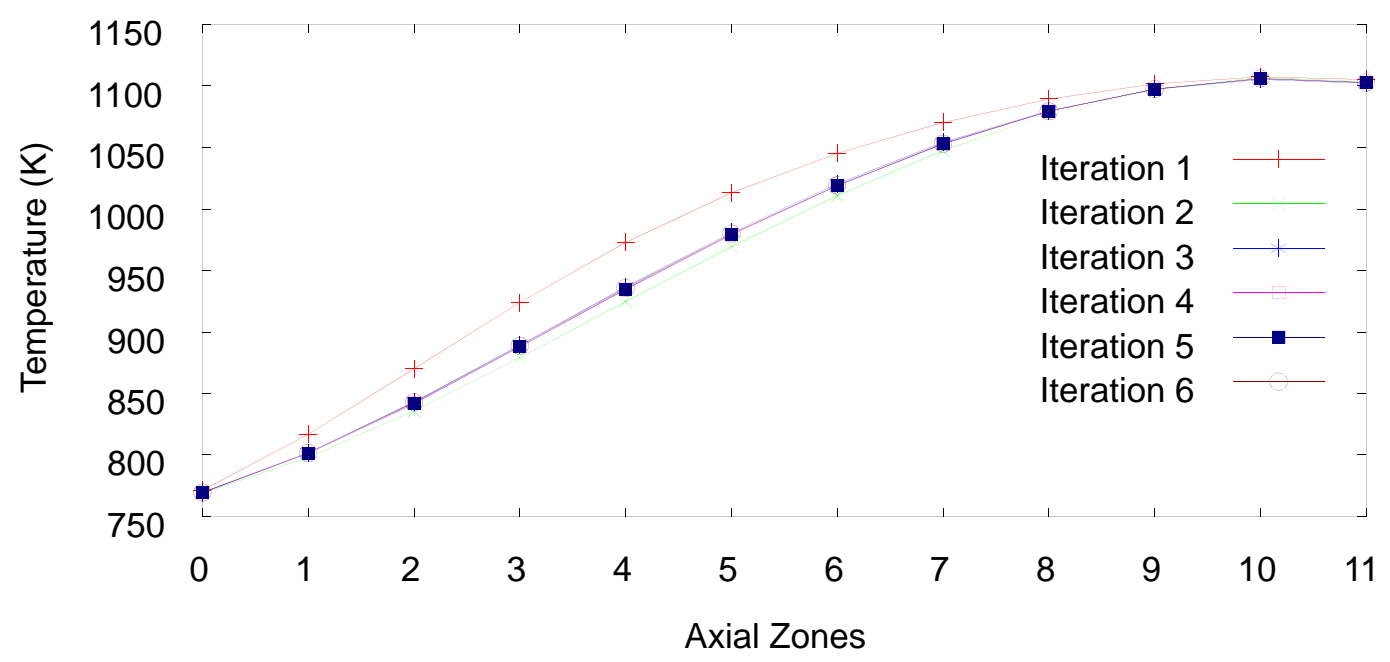

Temperatures in Axial Planes of the Mid Core Ring Zone, PSeudo Material Runs

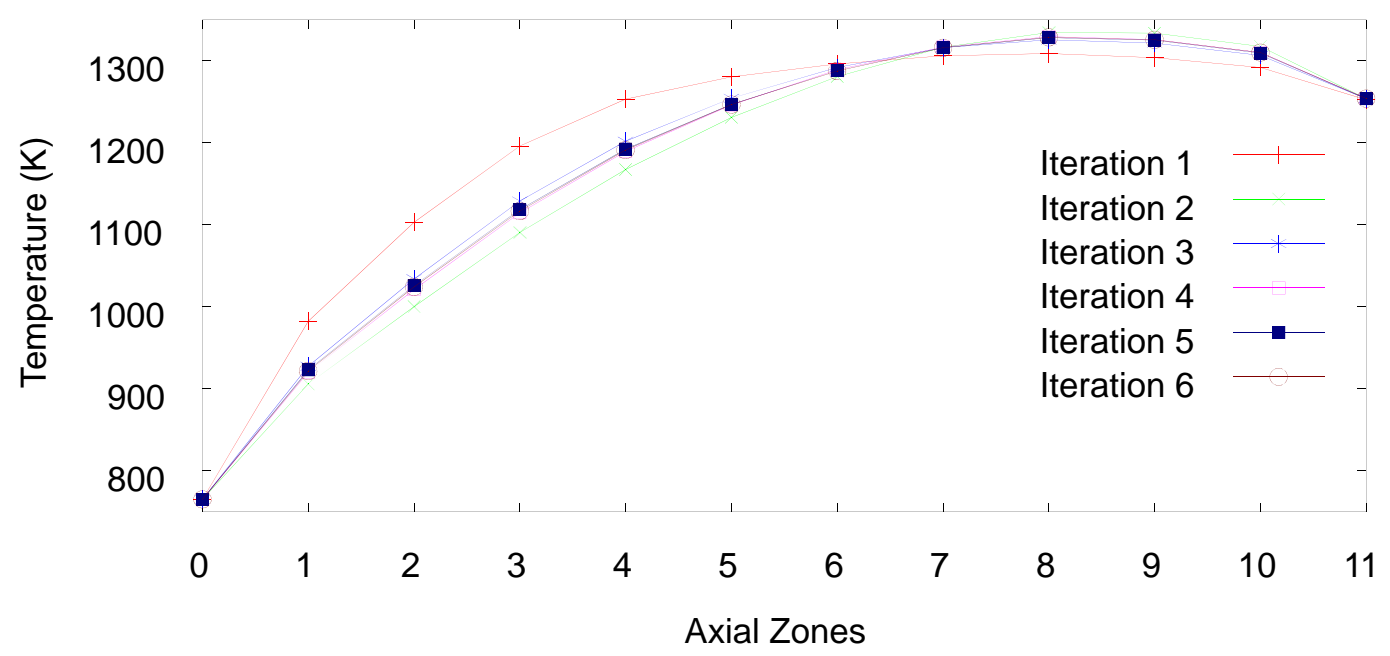

Temperatures in Axial Planes of the Mid Core Ring Zone, OTF Doppler Runs

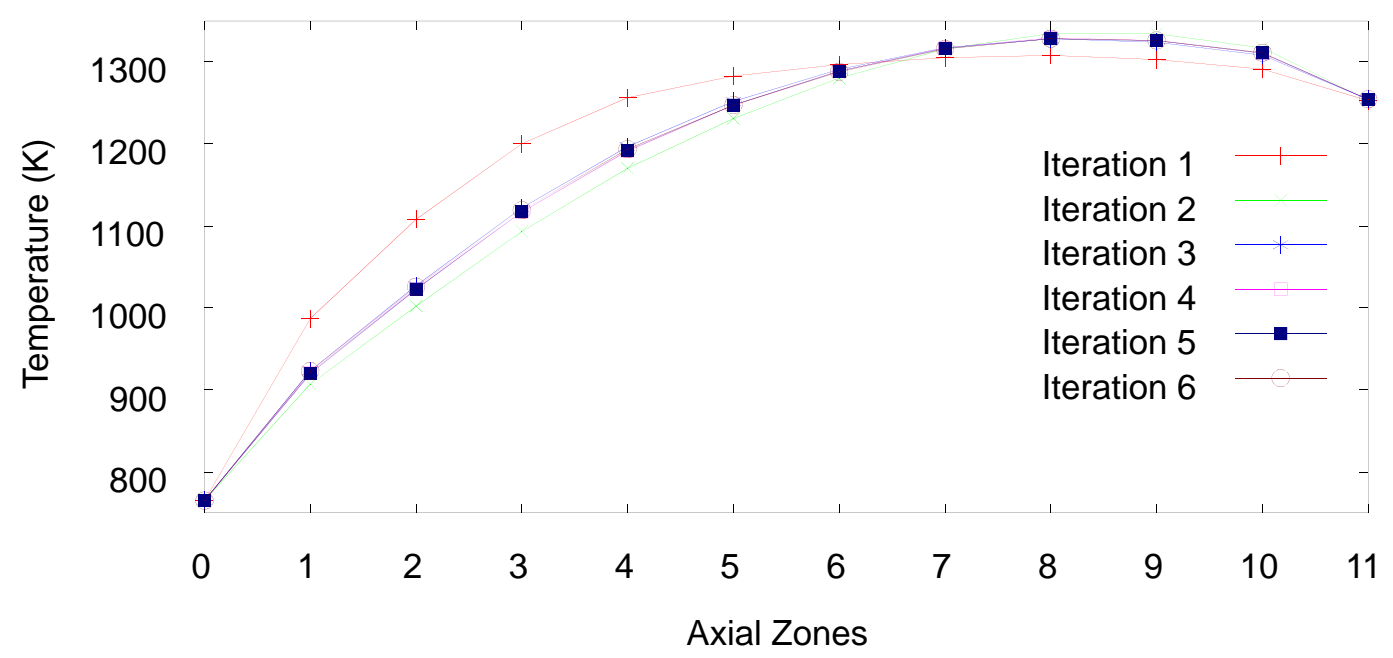


For both the reflector and the core, it is apparent from the figures that the OTF methodology leads to convergence as fast or faster than the pseudo material case. Indeed, RMS differences (across the axial planes) between iterations drop to below $0.8 \mathrm{~K}$ after iteration 5 for all five regions for the OTF runs, and to below $1.06 \mathrm{~K}$ for all five regions for the pseudo material runs after iteration 6 .

Agreement between the OTF and pseudo material with respect to temperature was also quite good. Shown in the figures below are the RELAP3D temperatures derived from the iteration 6 pseudo material simulation and the iteration 5 OTF Doppler simulations for each of the five regions. The left $\mathrm{y}$-axis gives the actual temperature, while the right $\mathrm{y}$-axis gives the difference between the OTF and pseudo material cases. RMS differences were less than $1.01 \mathrm{~K}$ and $0.1 \%$ for all five regions.

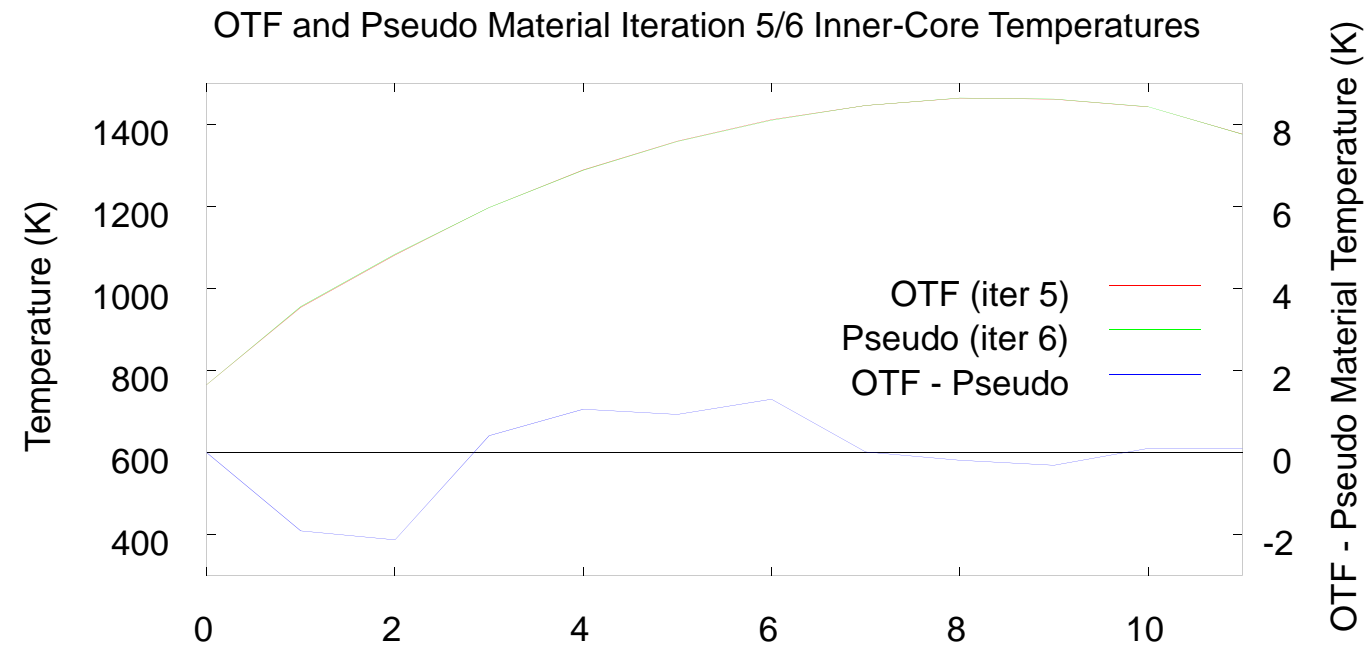

Axial Zone

OTF and Pseudo Material Iteration 5/6 Mid-Core Temperatures

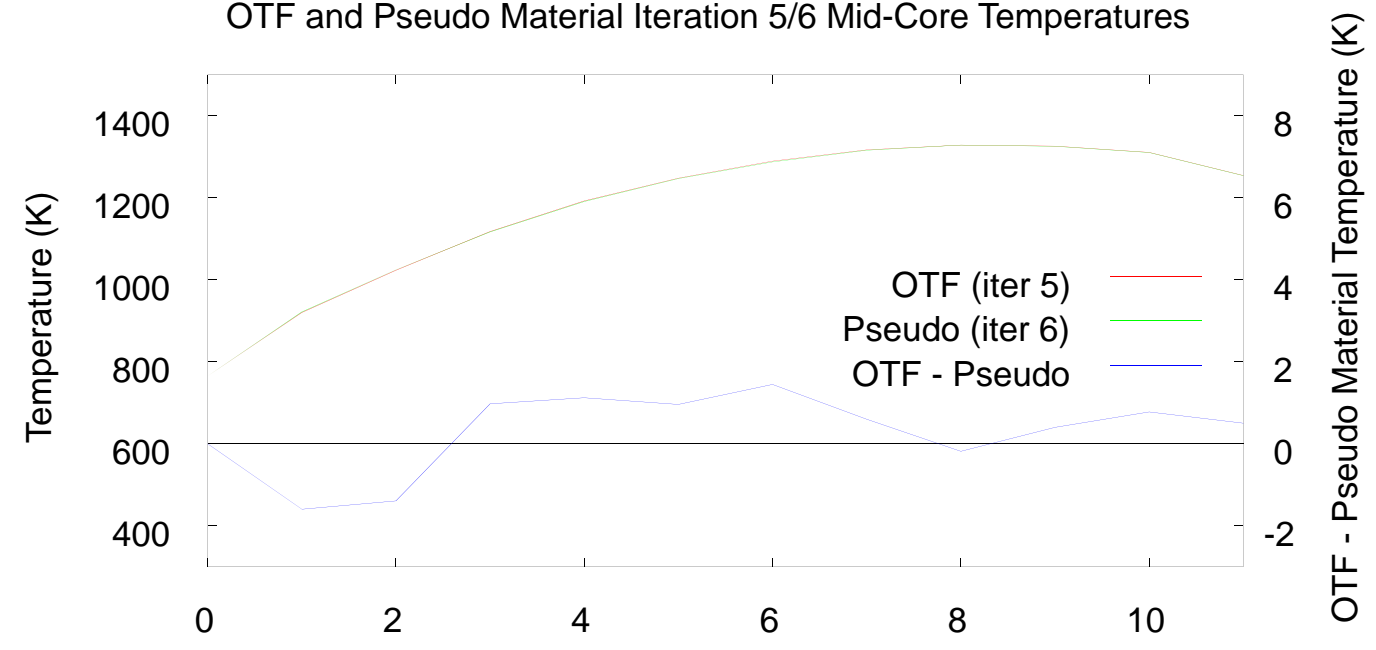

Axial Zone 


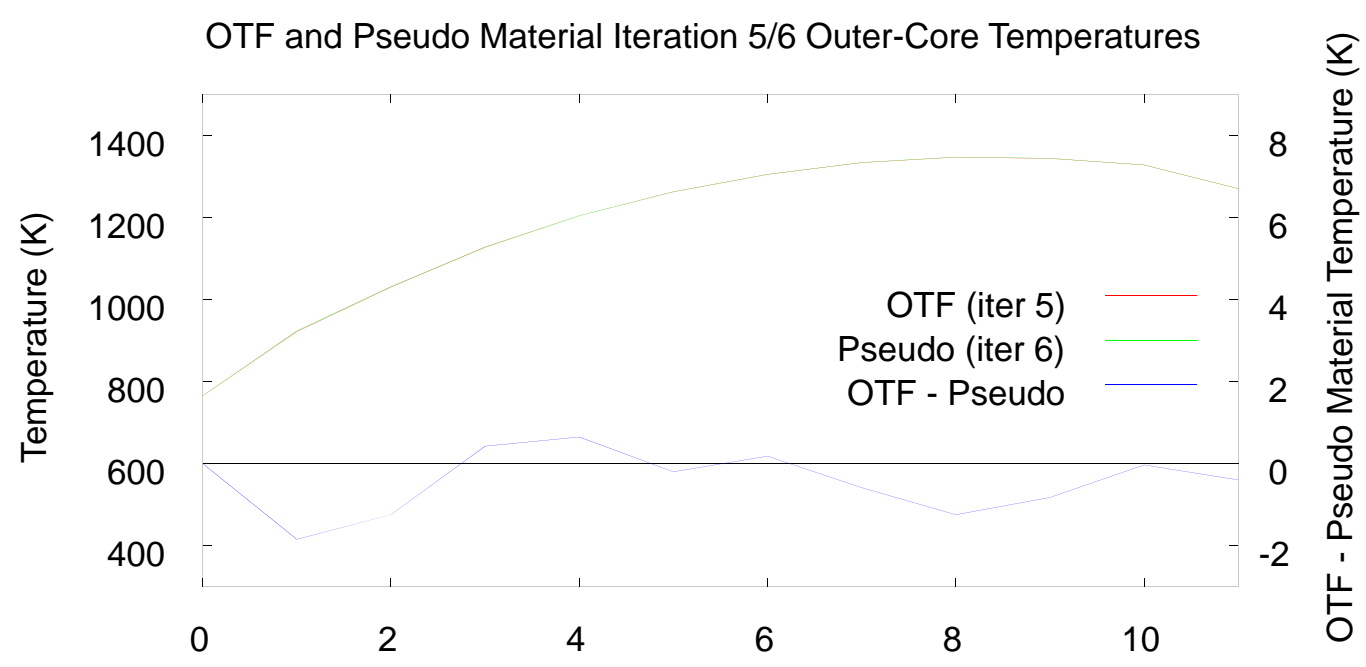

Axial Zone

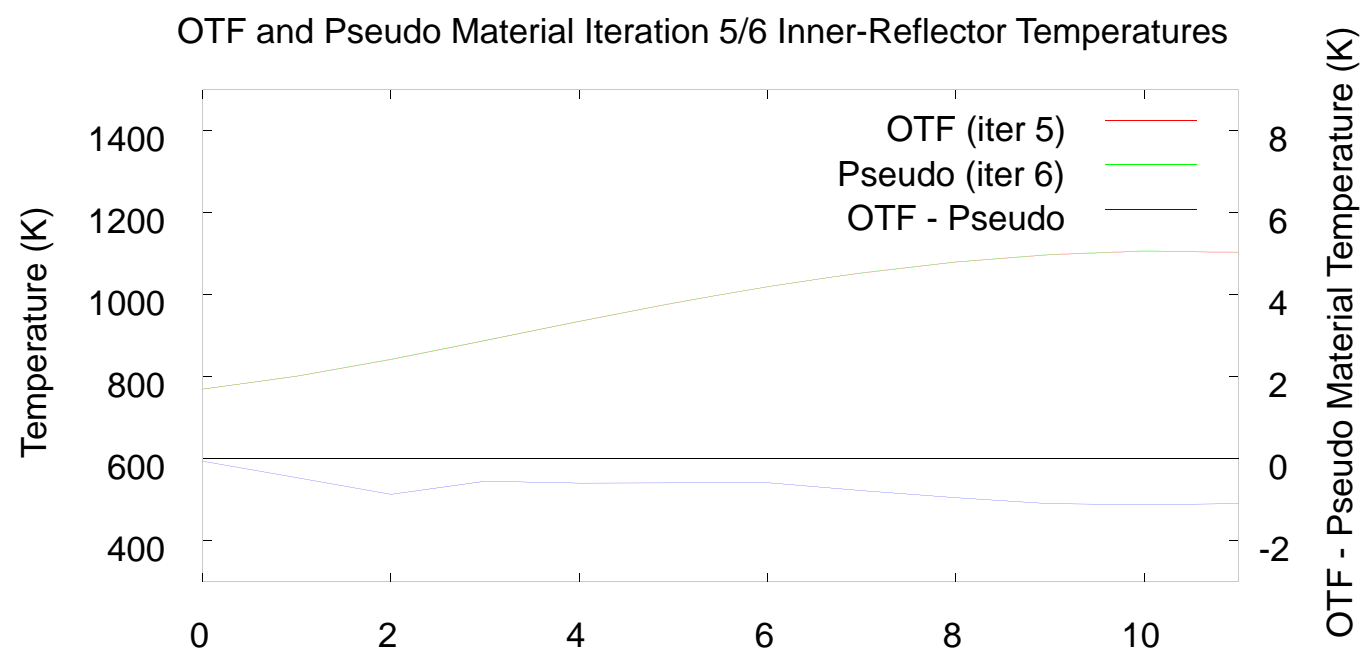

Axial Zone

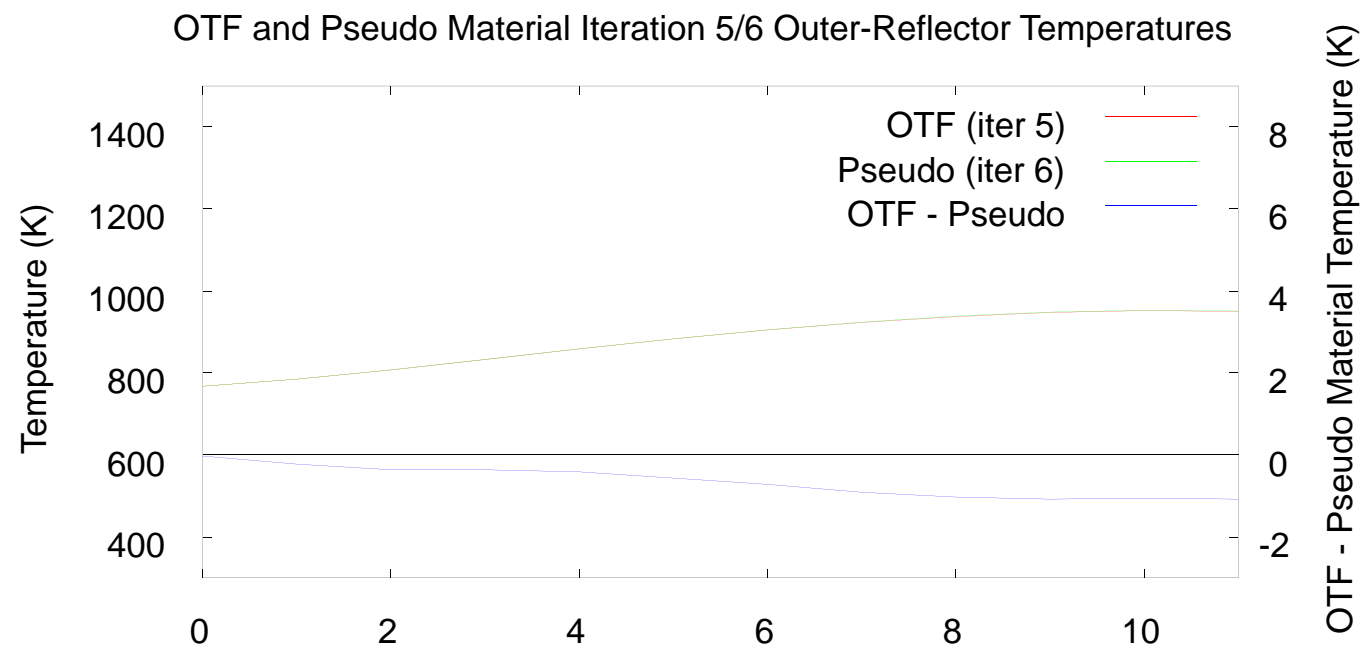

Axial Zone 
Unfortunately, in both cases subsequent iterations produced temperatures with larger RMS differences from the most recent iteration, with even larger divergences between the OTF and pseudo material temperatures. Indeed, the trajectory of the RMS difference across all axial planes in each region exhibited a sharp descent to minima but does not continue to converge with subsequent iterations. This is shown in the two figures below, which give the RMS differences in axial plane temperatures between iterations for all five regions for the OTF and pseudo material cases.

RMS Temperature Difference from Previous Iteration Over Axial Planes -- OTF Doppler

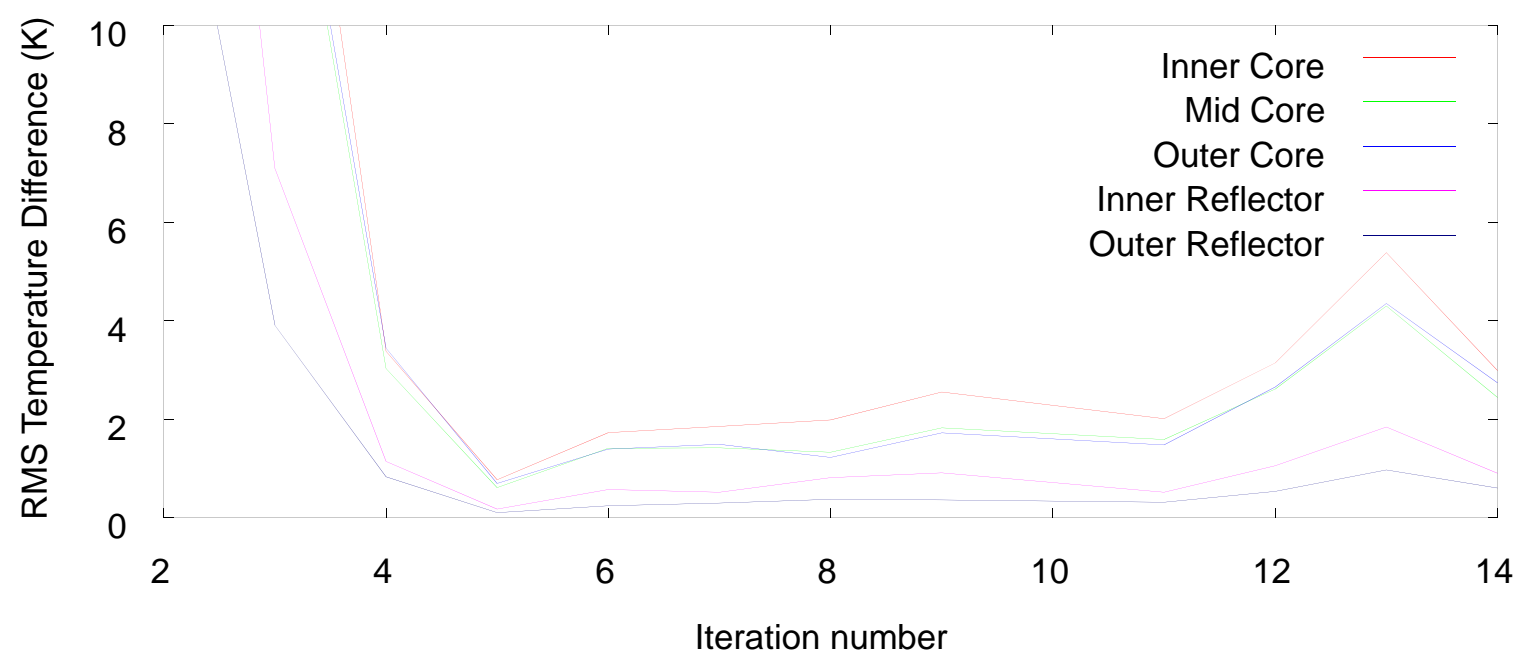

RMS Temperature Difference from Previous Iteration Over Axial Planes -- Pseudo Materials

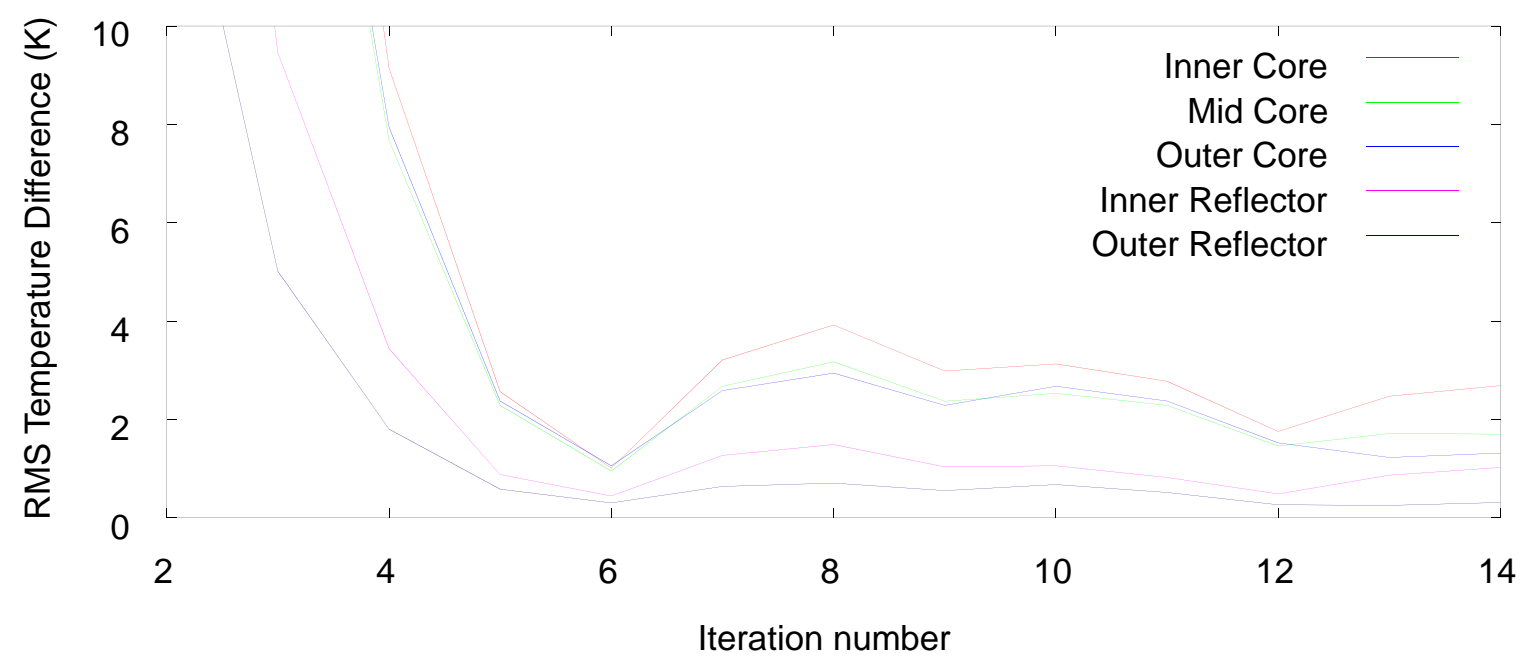

\section{Convergence:}

Assuming that the issue above was primarily due to uncertainty in the MCNP5 power distributions, additional runs were performed using 500K histories, starting with the iteration 6 RELAP3D output temperatures, as that when it seemed that k-effective was converged for both sets of runs. As expected, variation in k-effective with the additional histories was much smaller for both the OTF and pseudo 
material cases. However, after improving between iterations 6 and 7, RMS temperature differences between iterations began to exhibit a pattern similar to that seen in the figures above, and OTF and pseudo material results did not exhibit any trend toward convergence.

At that point, starting with the iteration 7 RELAP3D temperatures, under relaxation was imposed on the temperature updates between iterations. Iteration $8 \mathrm{MCNP}$ input temperatures were set to the weighted average (with relaxation parameter $\alpha$ of .5) of the iteration 7 MCNP input temperatures and the RELAP3D iteration 7 output temperatures. Results were significantly better than the iteration 8 results without relaxation, both in terms of RMS temperature differences between iterations for each of the two cases, and also in terms of agreement between the two sets of data. Relaxed iteration 8 results were not, however, as good as iteration 7 results. Two subsequent relaxed iterations were then performed, but with relaxation parameter reduced to .25. Agreement was excellent for iteration 9, with results converging to within $1.1 \mathrm{~K}$ (less than $0.1 \%$ ) of the iteration 8 temperatures for both cases, and also to within $0.84 \mathrm{~K}$ and $0.08 \%$ between the OTF and the pseudo material case. For iteration 10, however, while the OTF Doppler runs showed the same level of agreement with the OTF iteration 9 temperatures, the pseudo material temperatures began diverging, leading to larger differences between the OTF and pseudo material results than found in the prior iteration. A second iteration 10 computation was performed, using a larger value of $\alpha(0.75)$ in setting the input temperatures. Both the OTF and pseudo material RELAP3D temperatures derived from these new iteration 10 simulations agreed to within $0.75 \mathrm{~K}$ with their iteration 9 temperatures, and they agreed with each other to within $1.08 \mathrm{~K}$, as measured by RMS difference. This is just slightly worse than the iteration 9 agreement.

These results are shown in the following tables and figures. The first two tables show the RMS differences (over the axial planes in each region) between successive iterations for all five regions, first for the OTF Doppler case and then for the pseudo material case. The third table shows the RMS differences between the OTF temperatures and the pseudo material temperatures in each region for the various iterations.

\begin{tabular}{|c|c|c|c|c|c|}
\hline Iter, $(\alpha)$ & Inner Core & Mid Core & Outer Core & Inner Reflector & Outer Reflector \\
\hline $7(1.0)$ & .584 & .578 & .847 & .120 & .289 \\
\hline $8(0.5)$ & 1.54 & 1.27 & 1.24 & .540 & .276 \\
\hline $9(.25)$ & 1.04 & .786 & .934 & .316 & .210 \\
\hline $10(.25)$ & 1.06 & .927 & .938 & .346 & .215 \\
\hline $10(.75)$ & .734 & .600 & .523 & .292 & .124 \\
\hline
\end{tabular}

RMS temperature differences from previous iteration for regions in OTF Doppler simulations

\begin{tabular}{|c|c|c|c|c|c|}
\hline Iter, $(\alpha)$ & Inner Core & Mid Core & Outer Core & Inner Reflector & Outer Reflector \\
\hline $7(1.0)$ & 1.16 & .891 & .982 & .432 & .208 \\
\hline $8(0.5)$ & 2.01 & 1.56 & 1.59 & .760 & .359 \\
\hline $9(.25)$ & 1.09 & .904 & .940 & .342 & .217 \\
\hline $10(.25)$ & 2.40 & 1.92 & 2.05 & .974 & .511 \\
\hline $10(.75)$ & .432 & .399 & .456 & .126 & .064 \\
\hline
\end{tabular}

RMS temperature differences from previous iteration for regions in pseudo material simulations 


\begin{tabular}{|c|c|c|c|c|c|}
\hline Iter, $(\alpha)$ & Inner Core & Mid Core & Outer Core & Inner Reflector & Outer Reflector \\
\hline $7(1.0)$ & 1.10 & 1.08 & 1.07 & .881 & .586 \\
\hline $8(0.5)$ & 2.54 & 1.83 & 1.95 & 1.62 & .840 \\
\hline $9(.25)$ & .781 & .832 & .678 & .821 & .614 \\
\hline $10(.25)$ & 1.53 & 1.37 & 1.26 & .766 & .450 \\
\hline $10(.75)$ & .940 & .748 & .977 & 1.07 & .617 \\
\hline
\end{tabular}

Regional RMS temperature differences between OTF Doppler and pseudo material simulations

Several conclusions can be drawn from these results. First, while the OTF Doppler simulations do appear to be converging, it is unclear as to whether this is the case with the pseudo material runs. This is further illustrated in the five plots below, which give the iteration 10 temperatures for the OTF Doppler and pseudo material cases for each region, along with the differences between the OTF and pseudo material temperatures at iterations 10 and 7. For all five regions, the OTF iteration 10 temperatures are closer to the iteration 7 temperatures than for the pseudo material runs.

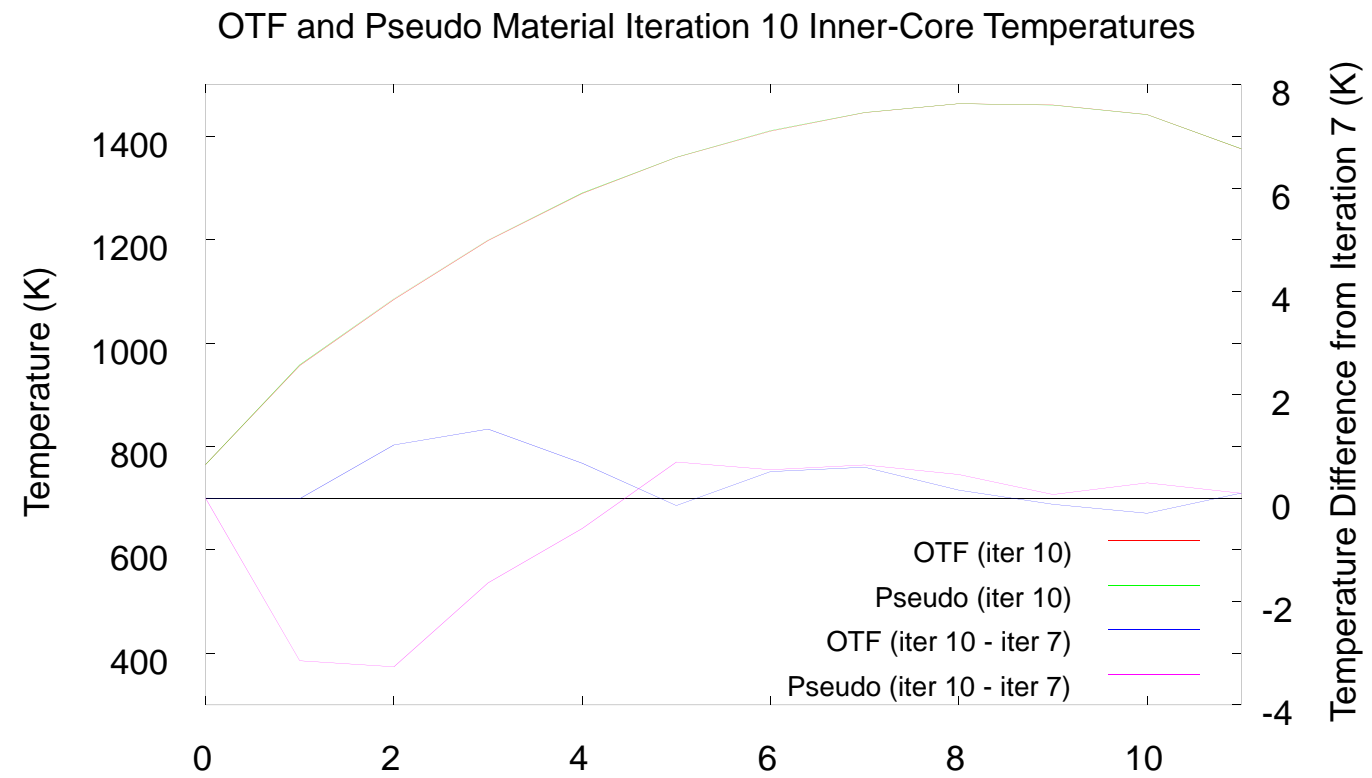

Axial Zone 


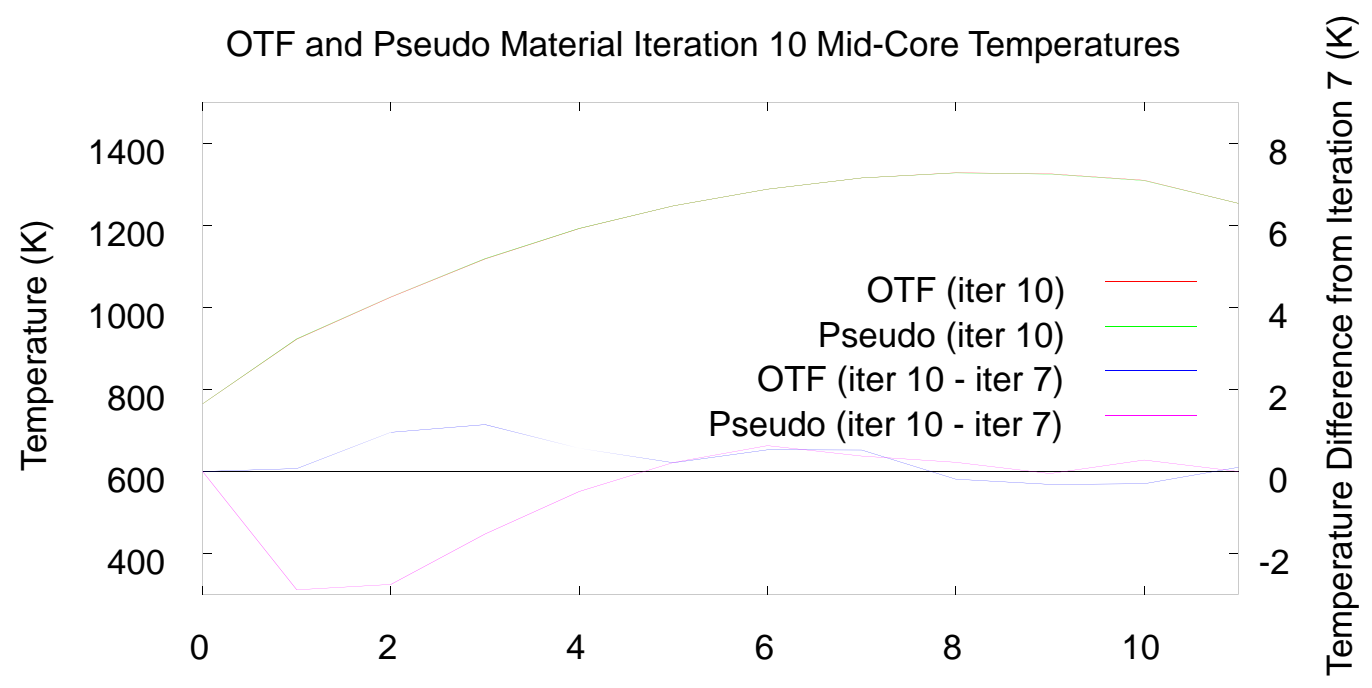

Axial Zone

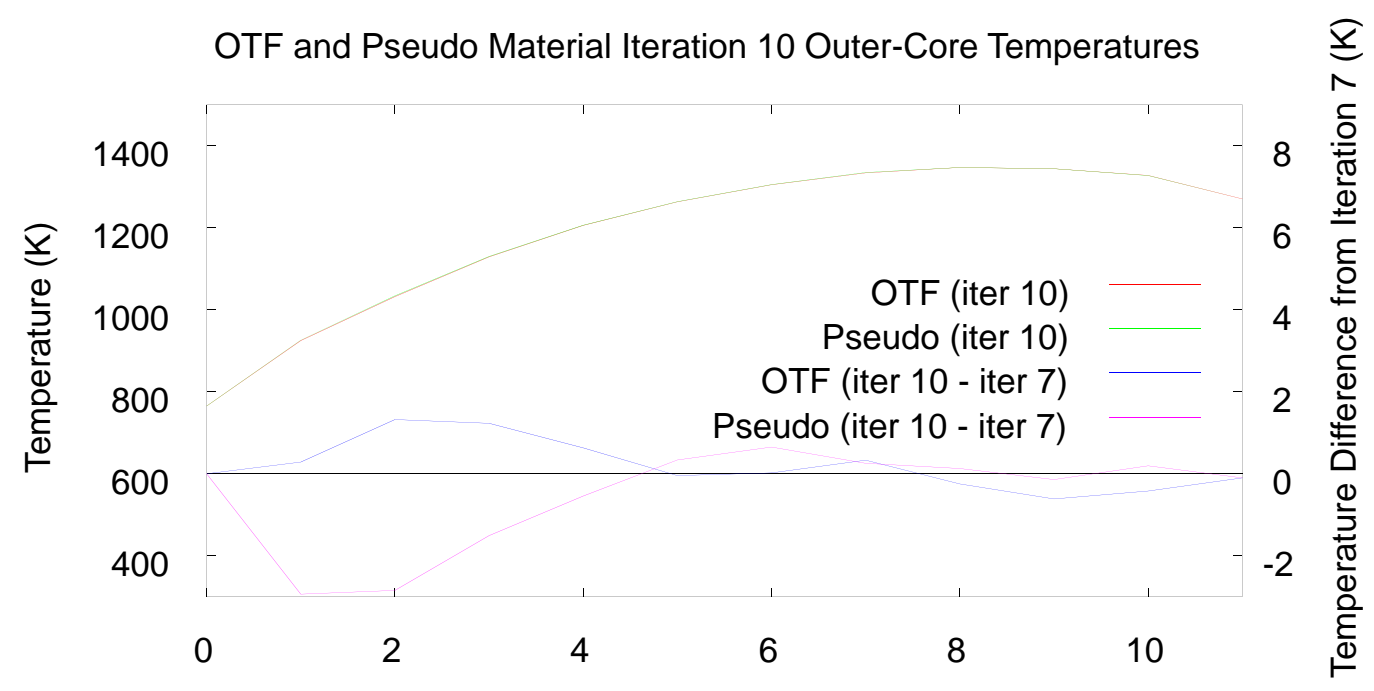

Axial Zone

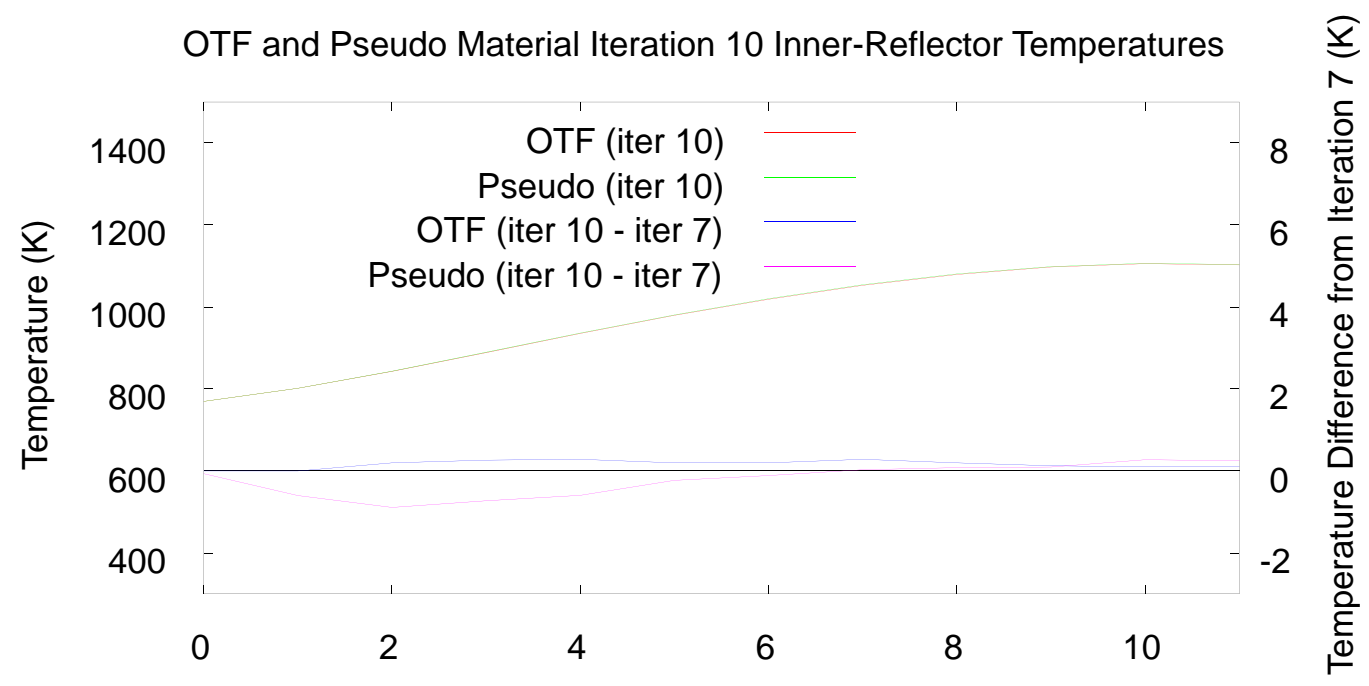

Axial Zone 


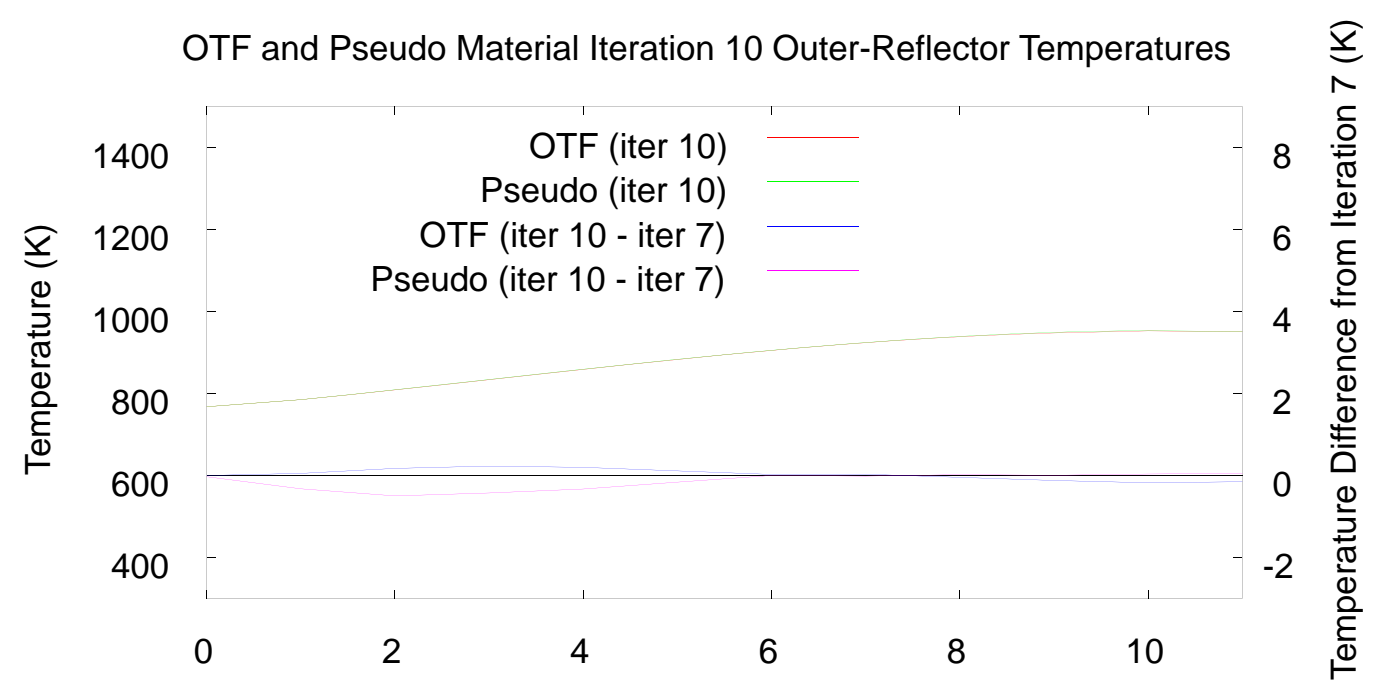

Axial Zone

The continuing variation in the pseudo material results at higher iterations most likely accounts for the slow convergence between the two cases. There are several possible explanations for the convergence issues with the pseudo material construct. First, the use of pseudo materials is an approximate method. Linearly scaling cross sections with temperature cannot yield exact cross section values for all isotopes in a given material, a restriction that does not encumber the OTF method. Also, as demonstrated by the improvement in convergence seen when increasing the number of histories per cycle, the statistical uncertainty in current problem may be too large to yield true convergence. Lastly, the errors in the convergence achieved for this problem of $0.1 \%$ is already equivalent to the tolerances set in developing the cross section data libraries. Pushing for even better convergence may not produce useful results. In any case, it is clear that the OTF Doppler methodology performs at least as well and probably better than the pseudo material method.

\section{Mosteller Doppler Coefficient Demonstration Problem}

Next, we explored the well-know Doppler Defect problem as posed by Mosteller [8]. In that problem, the Doppler reactivity coefficient is calculated for a cylindrical pin cell of infinite length with reflecting boundary conditions with the fuel at two different temperatures (600K and $900 \mathrm{~K})$. The Doppler coefficient is defined as the ratio of the change in 1/k-effective divided by the temperature difference (300K, in this case). A schematic of the cross section of the fuel pin (taken from Mosteller's paper) is shown in the figure below. The blue outer region is water with 1400 PPM of Boron.

In the appendix of the work cited, Mosteller provides the isotopic composition for the borated water and cladding at $600 \mathrm{~K}$, as well as UO2 and MOX fuel of varying enrichments at $600 \mathrm{~K}$ and $900 \mathrm{~K}$. 


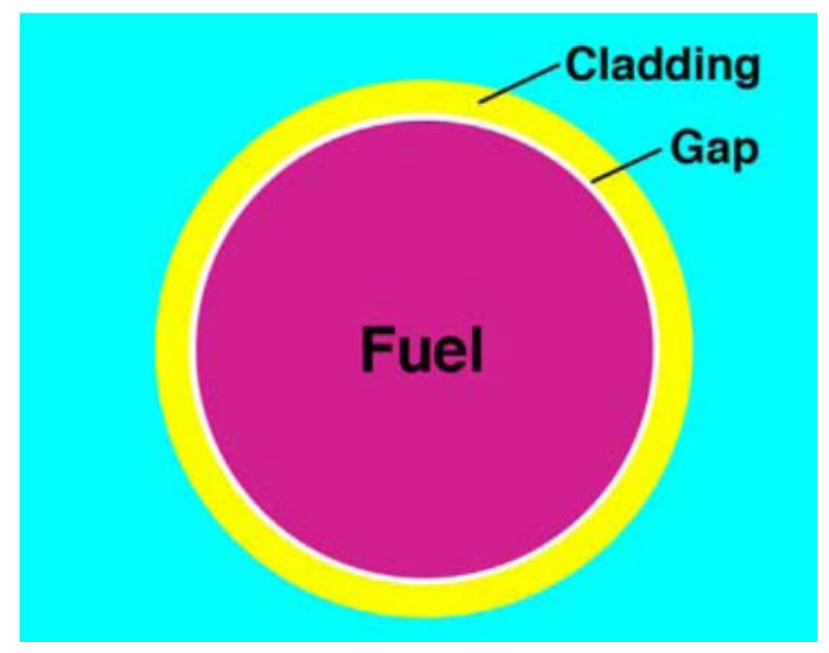

To demonstrate the utility of the OTF Doppler methodology and also to investigate the magnitude of the effect of assuming uniform hot (900K) temperatures in the fuel, we have repeated this benchmark but with the fuel subdivided into 10 concentric, equally spaced regions over which we allow the temperature to vary. If the temperature at the edge of the fuel is $600 \mathrm{~K}$ and the average temperature is $900 \mathrm{~K}$, it can be shown that the radial temperature distribution is given by $\mathrm{T}(\mathrm{r})=1200-600(\mathrm{r} / \mathrm{R})^{2}$, where $\mathrm{R}$ is the radius of the fuel. The average fuel temperatures in the 10 rings are given in the table below.

\begin{tabular}{|c|c|}
\hline Ring (inner to outer) & Average Temperature (K) \\
\hline 1 & 1197 \\
\hline 2 & 1185 \\
\hline 3 & 1160 \\
\hline 4 & 1125 \\
\hline 5 & 1077 \\
\hline 6 & 1016 \\
\hline 7 & 944 \\
\hline 8 & 861 \\
\hline 9 & 764 \\
\hline 10 & 656 \\
\hline
\end{tabular}

The only approximation imposed in moving from a single fuel region to 10 regions was that the atomic number densities in the fuel did not vary, and were instead equivalent to the average densities at 900K.

\section{Results}

Simulations were performed using 10000 cycles (9500 active) of 10000 histories each for constant fuel temperatures of $600 \mathrm{~K}$ and $900 \mathrm{~K}$ and varying temperatures from the table above for $\mathrm{UO}_{2}$ fuel with $\mathrm{U}$ 235 enrichments of .711, 1.6, 2.4, 3.1, 3.9, 4.5, and 5.0\%, and for reactor $\mathrm{MOX}$ fuel with $\mathrm{PuO}_{2}$ weight percents of 1.0, 2.0, 4.0, 6.0 and 8.0\%. Standard deviations in the calculated values of k-effective were on the order of 0.00006 (it was found that this level of precision was necessary to keep the error in the computed Doppler coefficients under 1\%). The results are shown in the figures below for the two fuel types. 
Doppler Coefficient vs. UO2 Wt \%

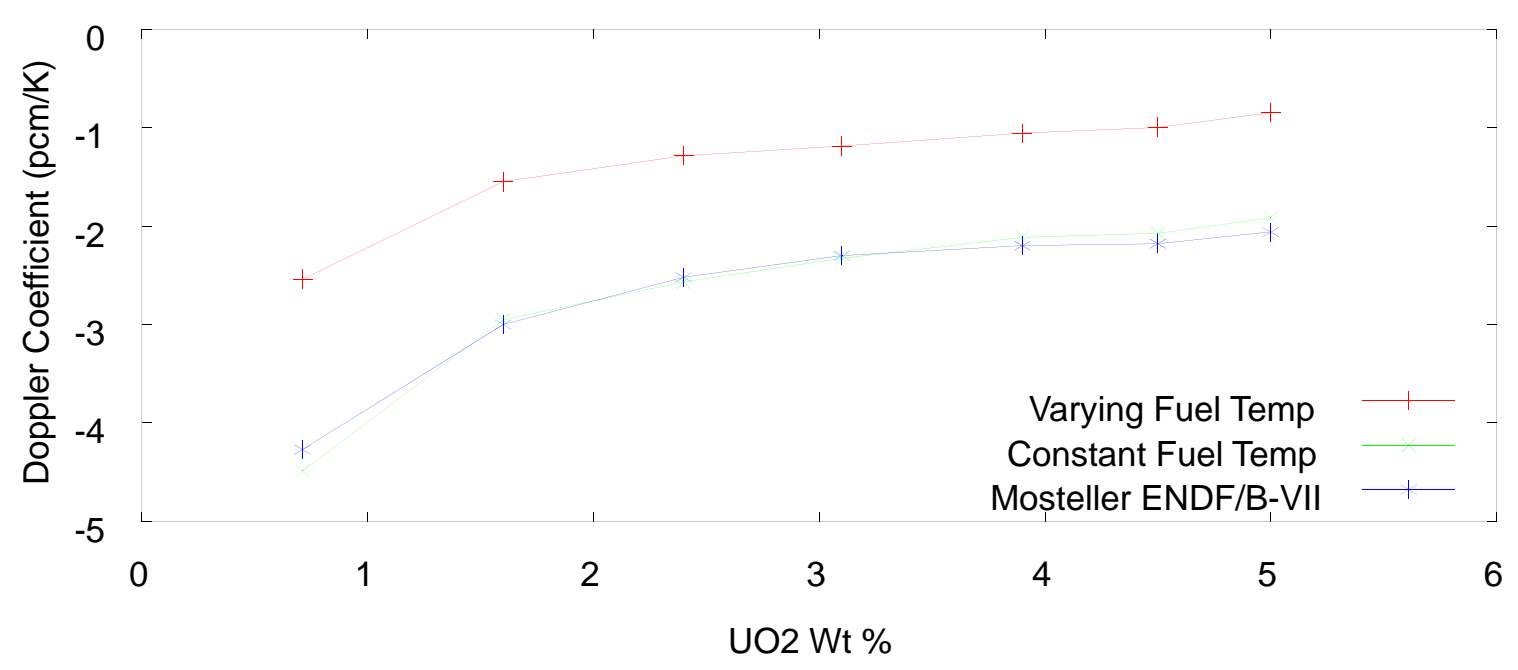

Doppler Coefficient for MOX Fuel vs. PuO2 Wt \%

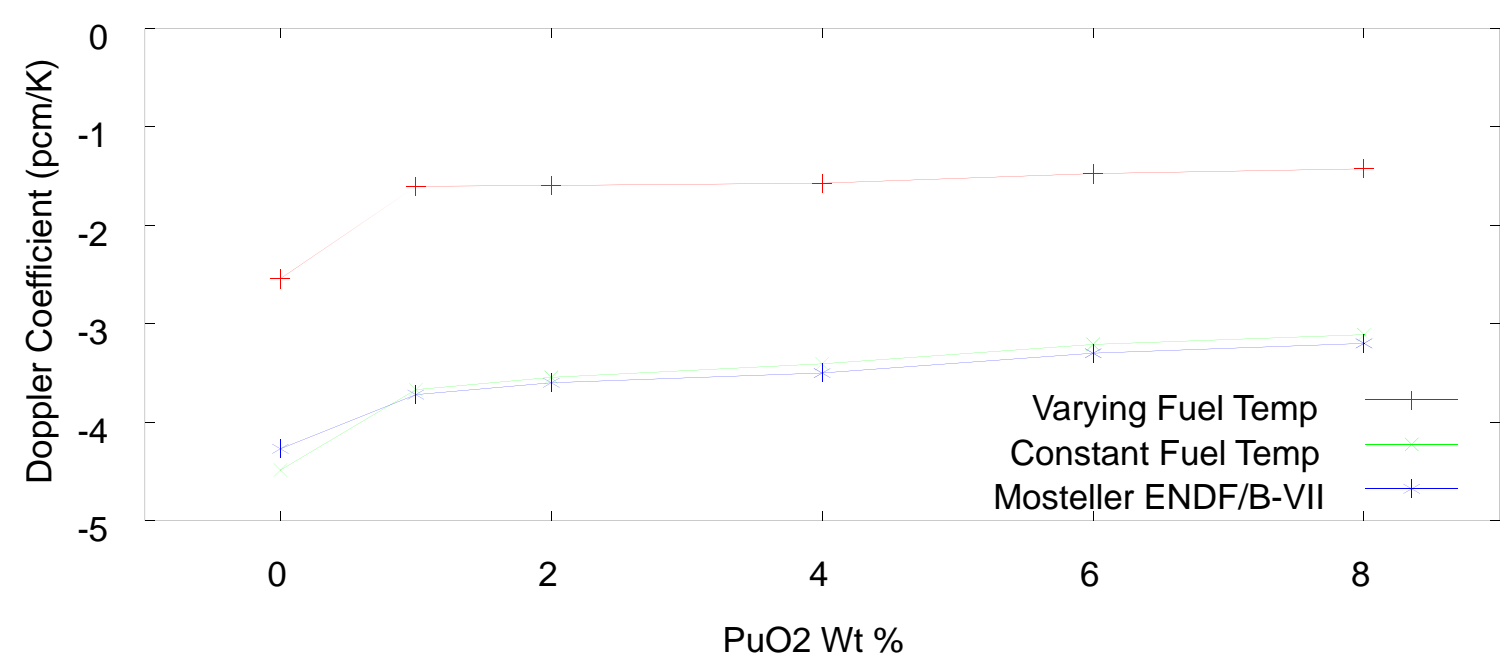

For both the $\mathrm{UO}_{2}$ and the MOX fuel, the constant temperature $(900 \mathrm{~K})$ results are consistent with those reported by Mosteller for ENDF7 cross sections.

What is clearly striking from the data is the magnitude of the effect of modeling the temperature variation in the fuel. In MOX fuel, the Doppler coefficients are less than one half of the values calculated assuming constant temperature fuel, and they show less variation with $\mathrm{PuO}_{2}$ weight. In $\mathrm{UO}_{2}$, the Doppler coefficients computed with radially varying fuel temperatures were again much lower in magnitude than constant temperature coefficients, but they showed more variation with enrichment than was found when constant fuel temperatures were assumed. 